\title{
CRISPRi screens in human astrocytes elucidate regulators of distinct inflammatory
} reactive states

Kun Leng ${ }^{1,2,3}, *$, Brendan Rooney ${ }^{1}$, Hyosung Kim ${ }^{4}$, Wenlong Xia ${ }^{5}$, Mark Koontz ${ }^{6}$, Mitchell Krawczyk $^{7,8}$, Ye Zhang ${ }^{8}$, Erik M. Ullian ${ }^{6}$, Stephen P.J. Fancy ${ }^{5}$, Matthew S. Schrag, ${ }^{9,10,11}$, Ethan S. Lippmann ${ }^{4,9,10,11}$, Martin Kampmann ${ }^{1,12,13,14, *}$

${ }^{1}$ Institute for Neurodegenerative Diseases, University of California, San Francisco, San Francisco, CA, USA

${ }^{2}$ Biomedical Sciences Graduate Program, University of California, San Francisco, San Francisco, CA, USA

${ }^{3}$ Medical Scientist Training Program, University of California, San Francisco, San Francisco, CA, USA

${ }^{4}$ Department of Chemical and Biomolecular Engineering, Vanderbilt University, Nashville, TN, USA Department of Neurology, Vanderbilt University, Nashville, TN, United States

${ }^{5}$ Departments of Neurology and Pediatrics, School of Medicine, University of California, San Francisco

${ }^{6}$ Department of Ophthalmology, School of Medicine, University of California, San Francisco

${ }^{7}$ Neuroscience Graduate Program, University of California, Los Angeles, Los Angeles, CA, USA

${ }^{8}$ Department of Psychiatry and Biobehavioral Sciences, David Geffen School of Medicine, University of California, Los Angeles, Los Angeles, CA, USA

${ }^{9}$ Department of Neurology, Vanderbilt University Medical Center, Nashville, TN, USA

${ }^{10}$ Vanderbilt Memory and Alzheimer's Center, Vanderbilt University Medical Center, Nashville, TN, USA

${ }^{11}$ Vanderbilt Brain Institute, Vanderbilt University, Nashville, TN, USA

${ }^{12}$ Weill Institute for Neurosciences, University of California, San Francisco, San Francisco, CA, USA

${ }^{13}$ Department of Biochemistry and Biophysics, University of California, San Francisco, San Francisco, CA, USA

${ }^{14}$ Chan Zuckerberg Biohub, San Francisco, CA, USA

*e-mail: kun.leng@ucsf.edu, martin.kampmann@ucsf.edu 


\begin{abstract}
In response to central nervous system injury or disease, astrocytes become reactive, adopting context-dependent states with altered functions. Certain inflammatory insults induce reactive astrocyte states that lose homeostatic functions and gain harmful outputs, and likely contribute to neuroinflammatory and neurodegenerative diseases. However, the cellular pathways controlling these states are not fully understood. Here, we combined single-cell transcriptomics with CRISPRi screening in human iPSC-derived astrocytes to systematically interrogate inflammatory reactivity. We found that autocrine-paracrine IL-6 and interferon signaling downstream of canonical NF- $\mathrm{\kappa B}$ activation drove two distinct inflammatory reactive states promoted by and inhibited by STAT3, respectively. Furthermore, these states corresponded with those observed in other experimental contexts, including in vivo, and their markers were upregulated in the human brain in Alzheimer's disease and hypoxic ischemic encephalopathy. These results and the platform we established have the potential to guide the development of therapeutics to selectively modulate different aspects of inflammatory astrocyte reactivity.
\end{abstract}

\title{
INTRODUCTION
}

Astrocytes perform critical homeostatic functions in the central nervous system (CNS), such as providing trophic support for neurons, regulating the formation and function of synapses, and maintaining the blood-brain barrier ${ }^{1}$. In CNS injury or disease, astrocytes respond to pathophysiological perturbations by becoming "reactive", which is defined as the adoption of context-specific cell states and associated alterations in morphology and function ${ }^{1,2}$.

Frequently, inflammatory processes play an important, if not central, role in the pathophysiology of CNS injuries, as seen in stroke ${ }^{3}$ and trauma ${ }^{4}$, and diseases such as multiple sclerosis ${ }^{5}$ and Alzheimer's disease ${ }^{6}$. As an immunocompetent CNS cell type, astrocytes actively participate in inflammatory signaling cascades and interact with both microglia, the CNS resident immune cells, as well as infiltrating immune cells from the periphery ${ }^{6,7}$. Proinflammatory cytokines, such as IL-1 $\alpha$, TNF, and C1q, induce reactive astrocytes that lose homeostatic functions while concurrently releasing factors that are potentially harmful in specific contexts ${ }^{8-10}$. From here on, we will refer to this form of astrocyte reactivity as "inflammatory reactivity".

Given that inflammatory astrocyte reactivity has been implicated in numerous neurodegenerative and neuroinflammatory diseases ${ }^{8,11}$, in addition to being associated with normal aging ${ }^{12}$, it is an attractive target for therapeutic development. However, the cellular pathways that control inflammatory astrocyte reactivity are still not fully understood, partly due to limitations in experimental scalability. On the one hand, animal models have provided key insights into genes required for astrocyte reactivity ${ }^{9,13,14}$, but the throughput to test genetic perturbations in vivo is extremely limited. On the other hand, in vitro astrocyte culture is a powerful alternative, but the scalability of experiments is still limited by the necessity to isolate primary astrocytes or the long duration of the differentiation process (up to 6 months) required to generate mature, non-reactive human iPSC (hiPSC)-derived astrocytes". Lastly, even though "omics" assays such as RNA-seq have been used extensively to identify cellular pathways altered in inflammatory reactive 
astrocytes $^{9,16}$, these assays by themselves can only provide correlative information and cannot uncover causal pathways controlling inflammatory reactivity.

Here, we developed a scalable method to generate hiPSC-derived astrocytes that allowed us to harness the power of pooled CRISPRi screening ${ }^{17}$ to systematically identify cellular pathways controlling inflammatory astrocyte reactivity. Following up on the top hits from CRISPRi screens with single-cell transcriptomics, we found that autocrine-paracrine IL-6 and interferon signaling drove two distinct inflammatory reactive states that were promoted by and inhibited by STAT3, respectively. Furthermore, we found that the inflammatory reactive states we identified corresponded to those observed in other experimental contexts, both in vitro and in vivo. Lastly, we also performed immunostaining supporting the existence of these inflammatory reactive states in human brains in the context of Alzheimer's disease and hypoxic-ischemic encephalopathy.

\section{RESULTS}

\section{iPSC-derived astrocytes (iAstrocytes) perform canonical astrocyte functions and sufficiently model inflammatory reactivity}

To generate hiPSC-derived astrocytes in a scalable manner, we modified the protocol from TCW et al. ${ }^{18}$ by inducing the expression of the gliogenic transcription factors NFIA and SOX9 ${ }^{19}$ during the differentiation process (see Methods). Our protocol resulted in astrocytes, which we will here on refer to as "iAstrocytes", with increased expression of astrocyte markers (Fig. 1a-b) as well as astrocyte-specific genes in general (Extended Data Fig. 1) compared to astrocytes generated using the original protocol from TCW et al. ${ }^{18}$ To confirm that iAstrocytes were capable of performing typical astrocyte functions such as maintaining the blood-brain barrier ${ }^{20}$ or promoting neuronal synapse maturation ${ }^{21}$, we co-cultured iAstrocytes with hiPSC-derived brain endothelial-like cells or neurons (see Methods). We found that brain endothelial-like cells cocultured with iAstrocytes displayed improved barrier formation and integrity compared to monoculture (Fig. 1c), demonstrating that iAstrocytes can promote the expected functional maturation of these cells. In addition, hiPSC-derived neurons (iNeurons) co-cultured with iAstrocytes developed synchronized calcium oscillations ${ }^{22,23}$ compared to iNeurons in mono-culture (Fig. 1d-f), demonstrating the ability of iAstrocytes to promote neuronal synapse maturation and consistent with the higher expression of genes encoding synapse-promoting proteins such as GPC4 ${ }^{24}$ and CADM1 ${ }^{25}$ in iAstrocytes compared to TCW astrocytes (Supplementary Table 1).

Having validated that iAstrocytes performed typical astrocyte functions, we next tested whether iAstrocytes could be used to model inflammatory astrocyte reactivity. It has been shown that the cytokines IL- $1 \alpha+\mathrm{TNF}+\mathrm{C} 1 \mathrm{q}$ induce inflammatory reactivity in vitro in both primary mouse astrocytes $^{8}$ as well as human cerebral organoid-derived astrocytes ${ }^{26}$. We found that treatment of iAstrocytes with IL- $1 \alpha+\mathrm{TNF}+\mathrm{C} 1 \mathrm{q}$ recapitulated previously reported in vitro phenotypes of inflammatory reactivity such as decreased phagocytosis of CNS substrates, decreased support of neuronal synapse maturation, and neurotoxicity ${ }^{8,26}$. Specifically, iAstrocytes displayed decreased phagocytosis of pHrodo-labeled synaptosomes after treatment with IL-1 $\alpha+$ TNF+C1q (Fig. 1gh). In addition, IL-1 $\alpha+\mathrm{TNF}+\mathrm{C} 1 \mathrm{q}$ treatment of iAstrocytes co-cultured with iNeurons abolished 
the development of synchronized neuronal calcium oscillations (Fig. 1d-f), demonstrating decreased support of neuronal synapse maturation by iAstrocytes. Finally, we found that the conditioned media from IL-1 $\alpha+\mathrm{TNF}+\mathrm{C} 1 \mathrm{q}$-treated iAstrocytes was toxic to iNeurons (Fig. i).

In addition to recapitulating key functional phenotypes of inflammatory reactivity, iAstrocytes also responded to $\mathrm{IL}-1 \alpha+\mathrm{TNF}+\mathrm{C} 1 \mathrm{q}$ at the transcriptomic level in a similar manner as primary mouse astrocytes and human iPSC-derived astrocytes generated using alternative protocols (Extended Data Fig. 2a-e, Supplementary Table 1). We noted, however, that the differentially expressed genes (DEGs) induced by IL-1 $\alpha+\mathrm{TNF}+\mathrm{C} 1 \mathrm{q}$ in hiPSC-derived astrocytes were not restricted to the previously defined "pan-reactive" and "A1" reactive categories (Extended Data Fig. 2c). This finding mirrored similar observations from Barbar et al. ${ }^{26}$, and supports the growing consensus that there is a multitude of distinct, context-specific reactive astrocyte states $^{26}$.

To find a cell-surface marker for inflammatory reactivity that is also functionally involved in neuroinflammation, we examined the DEGs induced by IL- $1 \alpha+\mathrm{TNF}+\mathrm{C} 1 \mathrm{q}$ in iAstrocytes. We found a dramatic increase in the transcript levels of VCAM1 (Fig. 1j), which encodes a celladhesion sialoglycoprotein known to facilitate the infiltration of peripheral immune cells into the central nervous system (CNS) during neuroinflammation ${ }^{27,28}$. We subsequently validated by flow cytometry that iAstrocytes indeed upregulated cell-surface VCAM1 after treatment with IL$1 \alpha+\mathrm{TNF}+\mathrm{C} 1 \mathrm{q}$ (Fig. 1k-1), corroborating previous reports in the literature demonstrating induction of VCAM1 in astrocytes under pro-inflammatory conditions ${ }^{29,30}$.

\section{iAstrocytes differentiated from CRISPRi hiPSCs maintain robust knockdown activity}

In order to investigate the effect of genetic perturbations on inflammatory reactivity in iAstrocytes, we generated iAstrocytes from hiPSCs stably expressing CRISPRi machinery (Fig. 2a), which enables specific and non-toxic knockdown of genes targeted by single guide RNAs (sgRNAs) delivered to the cell. We found that these iAstrocytes maintained robust CRISPRi activity, demonstrating $~ 100 \%$ knockdown of TFRC protein levels 6 days after lentiviral transduction with a single guide RNA (sgRNA) targeting TFRC compared to iAstrocytes transduced with a non-targeting control (NTC) sgRNA, regardless of whether the iAstrocytes were treated with IL-1 $\alpha+\mathrm{TNF}+\mathrm{C} 1 \mathrm{q}$ (Fig. 2b-c).

\section{CRISPRi screening and computational master regulator analysis uncover cellular pathways controlling inflammatory reactivity}

Having established CRISPRi in iAstrocytes, we aimed to systematically interrogate inflammatory reactivity in iAstrocytes with pooled CRISPRi screening ${ }^{17,31}$. We conducted screens based on two cell-autonomous phenotypes induced by IL- $1 \alpha+\mathrm{TNF}+\mathrm{C} 1 \mathrm{q}$ that can be analyzed by fluorescence activated cell sorting (FACS): decreased phagocytosis of pHrodolabeled synaptosomes (Fig. $1 \mathrm{~g}-\mathrm{h}$ ) and upregulation of cell-surface of VCAM1 (Fig. 1k-l) . For synaptosome phagocytosis, we performed the screens in both vehicle control-treated and IL$1 \alpha+\mathrm{TNF}+\mathrm{C} 1 \mathrm{q}-$ treated iAstrocytes to find genes whose knockdown could rescue or exacerbate 
the decrease in synaptosome phagocytosis induced by IL-1 $\alpha+\mathrm{TNF}+\mathrm{C} 1 \mathrm{q}$ (Fig. 3a). For upregulation of cell-surface VCAM1, we performed the screens in only IL-1 $\alpha+\mathrm{TNF}+\mathrm{C} 1 \mathrm{q}$-treated iAstrocytes (Fig. 3a) given that in the absence of cytokine treatment, very few iAstrocytes expressed VCAM1 (Fig. 11). To ensure that the results from the screens are generalizable, we validated that IL- $1 \alpha+\mathrm{TNF}+\mathrm{C} 1 \mathrm{q}$ also caused decreased synaptosome phagocytosis and upregulation of cell-surface VCAM1 in hiPSC-derived astrocytes generated using alternative protocols, as well as in primary mouse astrocytes (Extended Data Fig. 2f-i).

In addition to pooled CRISPRi screening, we also turned to computational master regulator analysis (MRA) $)^{32-34}$ as a method to uncover cellular pathways controlling inflammatory reactivity. The workflow of MRA consisted of first reconstructing a gene regulatory network between upstream regulators (e.g. transcription factors, or kinases and phosphatases) and target genes in human astrocytes based on coexpression, which required integrating a large number of human astrocyte expression profiles collected under diverse experimental conditions ${ }^{35}$ (see Methods); upstream regulators that control inflammatory reactivity were then predicted by examining the overlap between the regulons of upstream regulators with the DEGs induced by IL-1 $\alpha+\mathrm{TNF}+\mathrm{C} 1 \mathrm{q}$ in iAstrocytes (Fig. 3b).

We performed MRA using the human transcription factors ${ }^{36}$ (Fig. 3c) or kinases and phosphatases $^{37,38}$ (Fig. 3f) as upstream regulators, and similarly conducted pooled CRISPRi screening using a new custom sgRNA library targeting the human transcription factors (Fig. 3de) and a library targeting all kinases, phosphatases, and other genes representing the "druggable genome",39 (Fig. 3g-h). Together, these two approaches uncovered both expected regulators of inflammatory reactivity as well as potentially novel regulators.

For example, the top hits from both MRA and CRISPRi screens (Supplementary Tables 2-3) included the entire set of genes encoding all potential subunits of the NF- $\kappa \mathrm{B}$ transcription factor (REL, RELA, RELB, NFKB1, NFKB2; Fig. 3c-e) as well as genes encoding the upstream kinases of the canonical NF- $\kappa \mathrm{B}$ pathway (IKBKB, CHUK, IKBKG; Fig. $3 \mathrm{f}-\mathrm{h})^{40}$. For all of the above genes (with the exception of $N F K B 2$ ), the directionality of their screening phenotypes or master regulator analysis activity scores (see Methods) was consistent with the activation of the canonical NF-kB pathway being required for the induction of inflammatory reactivity. As an example, knockdown of RELA, which encodes the p65 subunit of the NF- $\mathrm{KB}$ transcription factor, blocked the induction of cell-surface VCAM1 (Fig. 3e) and rescued the decrease in phagocytosis (Fig. 3d) induced by IL-1 $\alpha+\mathrm{TNF}+\mathrm{C} 1 \mathrm{q}$. In addition, the activity score of RELA from MRA was positive, consistent with the positive average log-fold change of DEGs under the control of RELA (Supplementary Tables 2-3). For the case of NFKB2, knockdown of which actually increased VCAM1 induction by IL-1 $\alpha+\mathrm{TNF}+\mathrm{C} 1 \mathrm{q}$ (Fig. 3e), it is possible that the encoded protein, p100, may act to inhibit $R E L A$-dependent transcription through the cytoplasmic sequestration of $\mathrm{p} 65^{41}$.

Given that the canonical NF- $\kappa \mathrm{B}$ pathway is a well-studied master regulator of cellular responses to inflammatory stimuli ${ }^{42}$, its strong enrichment in the top hits from the screens and MRA serves as a positive control for the technical quality of the screens and the validity of the MRA pipeline. As a further control for the technical quality of the screens, we validated the phenotypes of selected top hits in independent experiments (Extended Data Fig. 3). 
In addition to the canonical NF- $\mathrm{KB}$ pathway, we also recovered genes involved in numerous other cellular pathways known to mediate inflammatory processes. One group of genes (STAT3, $C E B P B, C E B P D)$ consisted of transcription factors classically associated with the acute phase response in the context of systemic inflammation ${ }^{43,44}$. In the central nervous system, these transcription factors control similar responses in reactive astrocytes, such as the production of acute phase proteins $\mathrm{C} 3$ and $\alpha 1$-antitrypsin (encoded by the gene SERPINA1) by astrocytes challenged with IL-1 and $\mathrm{TNF}^{43}$ (also see Fig. $1 \mathrm{j}$ and Supplementary Table 1). Furthermore, STAT3 is required for the full induction of astrocyte reactivity caused by spinal cord injury ${ }^{13,45}$, ${ }^{46}$, and $C E B P B$ has been implicated in the pathogenesis of Alzheimer's disease (AD) ${ }^{47,48}$. We found that knockdown of STAT3, CEBPB, and CEBPD in the context of in vitro IL$1 \alpha+\mathrm{TNF}+\mathrm{C} 1 \mathrm{q}$ treatment surprisingly promoted the induction VCAM1 while having complex effects on phagocytosis (Fig. 3d,e,h).

Another group of genes consisted of those involved in the response to interferons (IRF1, STAT1, $I K B K E)^{49-51}$. For example, we found that knockdown of IRF 1 reduced VCAM1 induction by IL$1 \alpha+\mathrm{TNF}+\mathrm{C} 1 \mathrm{q}$. This is consistent with the fact that interferons have been shown to potentiate the induction of VCAM1 by inflammatory cytokines ${ }^{29,52}$.

Lastly, we found genes involved cellular pathways that have been relatively less studied in the context of inflammatory astrocyte reactivity. For example, we uncovered genes involved in or related to the mTOR pathway (MTOR, LAMTOR3, LATS2, FOXK1 $)^{53,54}$, the glucocorticoid receptor pathway $(N R 3 C 1)$, the actin cytoskeleton $(A R P C 3, A C T R 2)$, and also relatively uncharacterized genes such as FOXC2 and ZBTB7A.

Overall, the results from CRISPRi screening and MRA demonstrate that numerous cellular pathways regulate distinct aspects of inflammatory reactivity. Of particular interest to us was the fact that knockdown of the acute phase response-related genes $S T A T 3, C E B P B$, and $C E B P D$ increased the induction of VCAM1 by IL-1 $\alpha+\mathrm{TNF}+\mathrm{C} 1 \mathrm{q}$, whereas knockdown of the interferonrelated gene IRF 1 decreased VCAM1 induction. Since all of the above genes are known to be involved in inflammatory responses, their opposing phenotypes suggested to us that VCAM1 upregulation may only be capturing one particular aspect of inflammatory reactivity, i.e. that there may exist distinct inflammatory reactive states, one of which is marked by VCAM1 upregulation.

\section{CROP-seq reveals two distinct inflammatory reactive states}

To gain deeper insight into how regulators uncovered by CRISPRi screening and MRA may control distinct inflammatory reactive states, we turned to CROP-seq ${ }^{55}$. By coupling CRISPRi perturbations to single-cell transcriptomics, CROP-seq enables the recovery of perturbationassociated changes in gene expression from a pooled experiment. We selected 30 regulators (Extended Data Fig. 4b) that were strong hits from CRISPRi screening and MRA for pooled knockdown in iAstrocytes treated with vehicle control or IL-1 $\alpha+\mathrm{TNF}+\mathrm{C} 1 \mathrm{q}$, and then performed CROP-seq to characterize the effect of knockdown on IL- $1 \alpha+\mathrm{TNF}+\mathrm{C} 1 \mathrm{q}$-induced gene expression (see Methods). 
Before examining the effect of knocking down different regulators on inflammatory reactivity, we first focused on iAstrocytes transduced with non-targeting control (NTC) sgRNAs. In the absence of IL-1 $\alpha+\mathrm{TNF}+\mathrm{C} 1 \mathrm{q}$, NTC iAstrocytes partitioned largely by cell cycle in uniform manifold approximation projection (UMAP), with cluster 3 corresponding to dividing cells. A small fraction $(<10 \%)$ of endothelial-like and stromal-like cells were also present, corresponding to clusters 5 and 6, respectively (Fig. 4a, Extended Data Fig. 4a-c, Supplementary Table 4). Upon treatment with IL-1 $\alpha+\mathrm{TNF}+\mathrm{C} 1 \mathrm{q}$, NTC iAstrocytes partitioned into two distinct inflammatory reactive states corresponding to clusters 1 and 2, with an additional cluster (cluster 4) corresponding to cycling cells (Fig. 4a, Extended Data Fig. 4a-c, Supplementary Table 4).

Upon further examination of the two distinct inflammatory reactive states induced by IL$1 \alpha+\mathrm{TNF}+\mathrm{C} 1 \mathrm{q}$, we found that iAstrocytes in cluster 1 expressed markers related to the acute phase response such as $C 3, I L 6$, and $I L I B$, whereas iAstrocytes in cluster 2 expressed markers related to interferon signaling such as VCAM1, CXCL10, and $L_{T B}^{56}$ (Fig. 4b). Enrichment analysis of cluster 2 markers confirmed a strong, specific enrichment (i.e. unique to cluster 2 markers) for interferon-related pathways (e.g. "Interferon Gamma Response", "Interferon Alpha Response") and transcription factor regulons (e.g. STAT1, IRF1) (Fig. 4c, Supplementary Table 5). On the other hand, cluster 1 markers showed a specific enrichment for pathways and transcription factor regulons related to the mTORC1-HIF1 $\alpha / \mathrm{MYC}$ axis of metabolic control ${ }^{57}$ (e.g. "mTORC1 Signaling", "Hypoxia", "Myc Targets V1"; HIF1A, CEBPD ${ }^{58}$ ). For brevity, we will hereon refer to the inflammatory reactive astrocyte state corresponding to cluster 1 as "IRAS1" and the one corresponding to cluster 2 as "IRAS2".

Given that the results from CRISPRi screening and MRA pointed towards the canonical NF- $\kappa B$ pathway being required for the induction of inflammatory reactivity, we examined the effect of

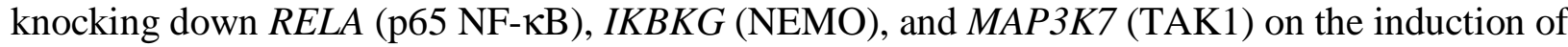
IRAS1 and IRAS2 by IL- $1 \alpha+\mathrm{TNF}+\mathrm{C} 1 \mathrm{q}$ in our CROP-seq data. Aligned UMAP embedding (see Methods) of RELA, IKBKG, and MAP3K7 knockdown iAstrocytes and NTC iAstrocytes showed that RELA knockdown abrogated the induction of both IRAS1 and IRAS2, with IKBKG and $M A P 3 K 7$ knockdown showing similar but less complete effects (Fig. 4d). Examining the expression of IRAS1 and IRAS2 markers in IL-1 $\alpha+\mathrm{TNF}+\mathrm{C} 1 \mathrm{q}-$ treated NTC iAstrocytes vs. IL$1 \alpha+\mathrm{TNF}+\mathrm{C} 1 \mathrm{q}$-treated $R E L A, I K B K G$, and $M A P 3 K 7$ knockdown iAstrocytes showed the same pattern (Fig. 4e). Lastly, using C3 and VCAM1 as markers of IRAS1 and IRAS2, respectively, we validated the above finding with flow cytometry in an independent experiment (Fig. 4f).

To gain a more global view of how knocking down different regulators affected the induction of inflammatory reactivity, we performed differential gene expression analysis for each regulator to find how regulator knockdown altered the differential expression induced by IL-1 $\alpha+\mathrm{TNF}+\mathrm{C} 1 \mathrm{q}$ (Extended Data Fig. 4d, Supplementary Table 6; see Methods). We then performed hierarchical clustering on the log-fold-changes (weighted by statistical significance) of the union of all DEGs (see Methods). We found that regulators segregated into three major groups, with one group (G1) consisting largely of regulators involved in the canonical NF- $\kappa \mathrm{B}$ pathway (RELA,IKBKG, $N F K B 1, R E L, C H U K)$ and upstream signal transduction (IRAK4, RIPK1, MAP3K7), another group $(\mathrm{G} 2)$ that contained acute phase response-related transcription factors $(C E B P B, C E B P D$, STAT3), and the last group (G3) consisting of regulators with weak knockdown phenotypes 
(Extended Data Fig. 4e). In terms of coordinately regulated genes, we recovered 10 gene modules that displayed distinct patterns across knockdown of different regulators (Extended Data Fig. 4e, Supplementary Table 7).

Of particular interest were modules M3, M4, and M9, which all had reduced induction upon knockdown of $\mathrm{G} 1$ regulators and thus appeared to be downstream of the canonical NF- $\mathrm{KB}$ pathway. Interestingly, M4 genes contained and had similar enrichments as IRAS1 markers (e.g. IL6; see Supplementary Table 7) and displayed reduced induction upon knockdown of G2 regulators, whereas M3 and M9 genes contained and had similar enrichments as IRAS2 markers (e.g. VCAM1; see Supplementary Table 7) and displayed increased induction upon knockdown of $\mathrm{G} 2$ regulators (Extended Data Fig. 5). This led us to hypothesize that the acute phase response-related transcription factors $C E B P B, C E B P D$, and STAT3 promoted IRAS1 while inhibiting IRAS2.

\section{IL-6 and interferons act in an autocrine-paracrine manner to drive IRAS1 and IRAS2}

Given that IRAS1 expressed markers related to the acute phase response, which is driven by IL-6 ${ }^{59}$, and that IRAS2 expressed markers related to the response to interferons, we hypothesized that IL-1 $\alpha+\mathrm{TNF}+\mathrm{C} 1 \mathrm{q}$ induced iAstrocytes to secrete IL-6 and interferons, which then acted on the iAstrocytes in an autocrine-paracrine manner. We found that iAstrocytes indeed secreted appreciable amounts of IL-6 ( 40,000 pg/mL) and interferons $(\sim 1,000 \mathrm{pg} / \mathrm{mL}$ of IFN- $\beta)$ in response to IL-1 $\alpha+\mathrm{TNF}+\mathrm{C} 1 \mathrm{q}$ (Fig. 5a). iAstrocytes also secreted appreciable amounts of GMCSF $(\sim 3,500 \mathrm{pg} / \mathrm{mL})$, which is known to act synergistically with IL-6 ${ }^{60}$, as well as CXCL10 $(\sim 30,000 \mathrm{pg} / \mathrm{mL})$, which is produced in response to interferons ${ }^{61}$.

To test whether the secreted IL-6 and interferons acted on the iAstrocytes in an autocrineparacrine manner, we knocked down their receptors and downstream transcription factors and measured the induction of IRAS1 and IRAS2 by performing flow cytometry on C3 and VCAM1. We found that knockdown of IL6R or IL6ST, which respectively encode the IL-6 receptor and its signal transducing partner gp130, decreased the induction of C3+ iAstrocytes; the induction of VCAM1 in iAstrocytes increased slightly for the case of IL6ST knockdown but did not change for IL6R knockdown (Fig. 5b). Knockdown of STAT3, which is activated downstream of IL-6 ${ }^{62}$, similarly decreased the induction of $\mathrm{C} 3+$ iAstrocytes while increasing the induction of VCAM1+ iAstrocytes (Fig. 5b).

On the other hand, concurrent knockdown of IFNAR1 and IFNAR2 (see Methods), which encode subunits of the type I interferon receptor, or IFNGRI and IFNGR2, which encode subunits of the type II interferon receptor, decreased the induction of both C3+ iAstrocytes and VCAM1+ iAstrocytes (Fig. 5b). Concurrent knockdown of STAT1 and STAT2, which are activated downstream of interferons ${ }^{63}$, also resulted in a decrease in the induction of both $\mathrm{C} 3+\mathrm{iAstrocytes}$ and VCAM1+ iAstrocytes (Fig. 5b).

As an alternative approach to assay the induction of IRAS1 and IRAS2, we assayed the production of GM-CSF and CXCL10, which should be produced by IRAS1 and IRAS2 iAstrocytes respectively on the basis of mRNA expression (Fig. 5c). Knockdown of IL6ST or 
STAT3 decreased the production of GM-CSF but increased the production of CXCL10, whereas knockdown of IFNAR1/2 or STAT1/2 decreased the production of both GM-CSF and CXCL10 (Fig. 5d).

In addition to perturbing the response of iAstrocytes to autocrine-paracrine IL-6 and interferons, we also aimed to perturb transcription factors that we hypothesized to control the production of IL-6 and interferons, such as $\mathrm{CEBPB}^{64}$ and $\mathrm{IRF}{ }^{49}$, respectively. In these experiments, we stained for VCAM1 and C3 simultaneously (see Methods) to better assess the relative balance of IRAS1 and IRAS2. We noted that IL-1 $\alpha+\mathrm{TNF}+\mathrm{C} 1 \mathrm{q}$ induced some iAstrocytes that were both VCAM1+ and C3+ (Fig. 5e), consistent with the fact that in our single-cell data, C3 and VCAM1 expression was enriched but not exclusive to IRAS1 and IRAS2, respectively. We found that knockdown of $C E B P B$ increased the induction of VCAM1+/C3- and VCAM1+/C3+ iAstrocytes while decreasing the induction of VCAM1-/C3+ iAstrocytes, whereas knockdown of IRF 1 did not dramatically change the induction of VCAM1+/C3- or VCAM1-/C3+ iAstrocytes but decreased the induction of VCAM1+/C3+ iAstrocytes (Fig. 5e). Furthermore, we found that $N F K B 2$ knockdown phenocopied $C E B P B$ knockdown (Fig. 5f), suggesting a potentially novel regulation of $C E B P B$ by $N F K B 2$.

To summarize our findings so far, we created a model of how IL- $1 \alpha+\mathrm{TNF}+\mathrm{C} 1 \mathrm{q}$ treatment induces IRAS1 and IRAS2 through a primary NF- $\kappa \mathrm{B}$-dependent transcriptional response followed by autocrine-paracrine IL-6 and interferon signaling feedback loops (Fig. 5g): $C E B P B / D$ and $S T A T 3$ drive the induction of IRAS1 while inhibiting the induction of IRAS2 through the IL-6 loop, whereas STATI/2 and IRF1 promote the induction of both IRAS1 and IRAS2 through the interferon loop.

\section{IRAS1 and IRAS2 correspond to reactive astrocyte states generalizable across different species and disease contexts}

To test if IRAS1 and IRAS2 correspond to inflammatory reactive astrocyte states generalizable across different experimental paradigms of neuroinflammation, we integrated the NTC sgRNAtransduced iAstrocytes from our single-cell RNA-seq data to previously published astrocyte single-cell RNA-seq datasets using the anchor-based data integration functionality of Seurat ${ }^{65}$ (see Methods).

In Barbar et al. ${ }^{26}$, mixed cultures of neurons and glia derived from dissociation of human cerebral organoids were treated with vehicle control or IL-1 $\alpha+\mathrm{TNF}+\mathrm{C} 1 \mathrm{q}$. After isolating astrocytes from the Barbar et al. dataset and integrating them with NTC iAstrocytes from our study (see Methods), we found that a subset of IL-1 $\alpha+\mathrm{TNF}+\mathrm{C} 1 \mathrm{q}$-treated astrocytes from Barbar et al. co-clustered with IRAS1 and IRAS2 iAstrocytes (Fig. 6a-c). While Barbar et al. astrocytes co-clustering with IRAS1 iAstrocytes expressed IRAS1 markers such as IL6, CXCL2, and CXCL5, those co-clustering with IRAS2 iAstrocytes expressed IRAS2 markers such as CXCL10, IFIT3, and ISG15 (Fig. 6d; Extended Data Fig. 6e; Supplementary Table 8). Furthermore, the DEGs between IRAS2 vs. IRAS1 iAstrocytes overlapped strongly with the DEGs between the corresponding co-clustering Barbar et al. astrocytes (Extended Data Fig. 6a). 
In Wheeler et al. ${ }^{66}$, astrocytes were isolated by FACS from mice induced with experimental autoimmune encephalomyelitis (EAE), a widely used model of multiple sclerosis (MS), at different stages of EAE progression (naïve, acute, and remitting). After removing low-quality astrocytes from the Wheeler et al. dataset and integrating the remaining astrocytes with NTC iAstrocytes from our study (see Methods), we found that the vast majority of Wheeler et al. astrocytes from the acute stage of EAE co-clustered with IRAS1 and IRAS2 iAstrocytes (Fig. 6e-g) and also correspondingly expressed IRAS1 markers such as Srgn, Cxcl2, and Illb or IRAS2 markers such as Cxcl10, Ifit3, and Isg15 (Fig. 6h; Extended Data Fig. 6f; Supplementary Table 8). Overlap analysis of the DEGs between IRAS2 vs. IRAS1 iAstrocytes and the DEGs between the corresponding co-clustering astrocytes from Wheeler et al. also showed good agreement (Extended Data Fig. 6b).

Lastly, in Hasel et al. ${ }^{52}$, astrocytes were isolated by FACS from mice injected peripherally with saline or lipopolysaccharide (LPS), which induces acute neuroinflammation similar to that caused by sepsis. After removing non-astrocyte cells from the Hasel et al. dataset, integration of the Hasel et al. astrocytes with NTC iAstrocytes from our study showed that a subset of Hasel $e t$ $a l$. astrocytes from LPS-injected mice co-clustered with IRAS1 and IRAS2 iAstrocytes (Fig. 6i-k) and also correspondingly expressed IRAS1 markers such as $C 3, \mathrm{Cxcl5}$, and Tnfaip2 or IRAS2 markers such as Cxcl10, Ifit3, and Isg15 (Fig. 61; Extended Data Fig. 6g; Supplementary Table 8). Overlap analysis of the DEGs between IRAS2 vs. IRAS1 iAstrocytes and the DEGs between the corresponding co-clustering astrocytes from Hasel et al. further supported this correspondence (Extended Data Fig. 6c).

In addition to single-cell RNA-seq datasets, we also reanalyzed bulk RNA-seq data from Anderson et al. ${ }^{45}$, where astrocyte-specific RNA was purified from wild-type or astrocytespecific Stat 3 conditional knockout (cKO) mice subject to spinal cord injury (SCI). We found that Stat3-dependent genes (i.e. genes with lower expression in Stat3 cKO SCI vs. WT SCI; e.g. C3) tended to be highly expressed in IRAS1 iAstrocytes (Extended Data Fig. 6h), whereas Stat3repressed genes (i.e. genes with higher expression in Stat3 cKO SCI vs. WT SCI; e.g. Iigp1) were enriched for genes involved in the response to interferons (Extended Data Fig. 6i), similar to IRAS2 markers (see Supplementary Table 9 for Stat 3 cKO-related DEGs from Anderson et $a l$.) . Thus, the results from Anderson et al. not only further corroborate the existence of in vivo inflammatory reactive astrocyte states similar to IRAS1 and IRAS2, but also the regulatory role of STAT3 in inducing IRAS1 and inhibiting IRAS2 that we have proposed.

\section{Markers of IRAS1 and IRAS2 are upregulated in astrocytes in human disease}

Given that IRAS1 and IRAS2 corresponded to inflammatory reactive states found in various experimental paradigms of neuroinflammation, we reasoned that they would be present in the human brain as well under inflammatory conditions. In Alzheimer's disease (AD), neuroinflammation mediated by microglia and astrocytes contributes substantially to disease progression $^{67}$. We performed immunostaining of C3 and VCAM1, markers of IRAS1 and IRAS2 respectively, in post-mortem brain tissue derived from AD cases vs. age-matched controls (see Supplementary Table 10 for clinical metadata). We found a significant increase in the abundance of $\mathrm{C} 3+$ astrocytes in $\mathrm{AD}$ (Fig. 7a-b), consistent with previous studies ${ }^{8}$. Notably, C3+ astrocytes 
tended to be localized near $\mathrm{C} 3+$ plaques (Fig. 7a), which we deduced to be amyloid plaques given the fact that they are known to accumulate complement proteins such as $\mathrm{C} 3{ }^{68}$. Amyloid plaques are also known to accumulate SERPINA ${ }^{69}$, which is upregulated in IRAS1 reactive astrocytes and also in disease-associated reactive astrocytes found in mouse models of $\mathrm{AD}^{70}$. As for VCAM1, we did not observe a statistically significant difference in the abundance of VCAM1+ astrocytes between AD cases vs. controls (Fig. 7c-d). Lastly, C3+ astrocytes tended to be VCAM1- (118 VCAM1- vs. 1 VCAM1+ astrocytes among C3+ astrocytes aggregated across all individuals; Fig. 7a), and VCAM1+ astrocytes tended to be $\mathrm{C} 3-(13 \mathrm{C} 3-$ vs. $1 \mathrm{C} 3+$ astrocytes among VCAM1+ astrocytes aggregated across all individuals; Fig. 7c).

In addition to $\mathrm{AD}$, we also examined tissue derived from human neonatal hypoxic-ischemic encephalopathy (HIE), in which neuroinflammation contributes significantly to neuronal injury and negative neurologic sequela ${ }^{71}$. We observed increased abundance of both C3+ and VCAM1+ astrocytes in HIE (Fig. 7e-h).

\section{DISCUSSION}

Inflammatory reactive astrocytes have been implicated in numerous neurodegenerative and neuroinflammatory diseases such as Alzheimer's disease, Parkinson's disease, Huntington's disease, amyotrophic lateral sclerosis, and multiple sclerosis ${ }^{8,11}$. However, we do not yet have a full understanding of the cellular pathways controlling inflammatory reactivity in these contexts. Here, we employed pooled CRISPRi screening to systematically identify genes that control the response of hiPSC-derived astrocytes to the inflammatory reactivity-inducing cytokines IL$1 \alpha+\mathrm{TNF}+\mathrm{C} 1 \mathrm{q}$.

The scalability and homogeneity of our hiPSC-derived astrocytes ("iAstrocytes") was critical to our ability to perform pooled screens. However, it should be noted that iAstrocytes represent one of many available hiPSC-derived astrocyte models, and that there is a tradeoff between the scalability and maturation status of hiPSC-derived astrocytes. For our study, we found that iAstrocytes sufficiently modeled inflammatory reactivity, and we also ensured that the phenotypes we investigated were conserved across different hiPSC-derived astrocyte models.

Following up on the top hits from our CRISPRi screens with single-cell transcriptomics, we identified two distinct inflammatory reactive astrocyte states - "IRAS1" and "IRAS2" - driven by autocrine-paracrine IL-6 and interferon signaling. We found that STAT3 promoted IRAS1 while inhibiting IRAS2 through IL-6, whereas STAT1/2 and IRF1 promoted both IRAS1 and IRAS2 through interferons. Importantly, we found that IRAS1 and IRAS2 corresponded to inflammatory reactive astrocyte states found in other experimental contexts, both in vitro and in vivo. In light of the cellular pathways driving IRAS1 and IRAS2, we propose that IRAS1 be referred to in a general context as "STAT3-dependent reactivity"13,46, 72 and IRAS2 as "interferon-responsive reactivity" "52, 73, 74 .

It is likely that STAT3-dependent vs. interferon-responsive astrocyte reactivity have distinct functional outputs. Several studies already point towards potential functional outputs of STAT3dependent reactivity. For example, in Anderson et al. ${ }^{45}$, astrocyte-specific deletion of Stat3 was 
shown to prevent proper axon regeneration after spinal cord injury, suggesting that STAT3dependent reactivity may promote axon regeneration.

On the other hand, interferon-responsive reactivity may play an important role in EAE and MS. For example, Rothhammer et al. ${ }^{75}$ showed that inhibiting type I interferon signaling in astrocytes exacerbated the severity of EAE. Similarly, Hindinger et al. ${ }^{76}$ showed that inhibiting type II interferon in in astrocytes exacerbated the severity of clinical symptoms during peak disease. Furthermore, given that interferon-responsive reactive astrocytes upregulate VCAM1 and were shown to be adjacent to vasculature in Hasel et al. ${ }^{52}$, they may be important for controlling the trafficking of peripheral immune cells into the CNS parenchyma.

We believe that our work here will serve as a valuable resource for future work to further characterize the functional outputs of STAT3-dependent vs. interferon-responsive astrocyte reactivity. More generally, the approach pioneered here could be applied to uncover regulators of different reactive astrocytes states induced by other perturbations, which will pave the way for characterizing their functions and targeting them therapeutically.

\section{ACKNOWLEDGEMENTS}

We thank Emmy Li, Brandon Desousa, Indigo L. V. Rose, Vukasin Jovanovic, Zuzana Krejciova, and Nawei Sun for contributions to preliminary studies and discussions. We thank Professors Anna Molofsky, Aimee Kao, and Michael Oldham for serving on KL's thesis committee. We thank members of the Kampmann lab (Greg Mohl, Sydney Sattler, Olivia Teter) for discussions and feedback on the manuscript. We thank Brian Woo for cloning the transcription factors sgRNA library. This research was supported by NIH grant F30 AG066418 to KL, Chan Zuckerberg Initiative Ben Barres Early Career Acceleration Awards to ESL and M. Kampmann, NIH/NINDs grants (R01NS097551, P01NS083513, R21NS119954) to SF, and NIH grants P30 EY02162-39 and R03AG063157 to EMU. SF is a Harry Weaver Neuroscience Scholar of the National Multiple Sclerosis Society.

\section{AUTHOR CONTRIBUTIONS}

KL and M. Kampmann. conceptualized and led the overall project, and wrote the manuscript with input from all co-authors. KL performed the majority of experiments with support from BR and performed all data analysis. HK performed immunostaining of AD tissues provided by MSS and co-culture experiments with guidance from ESL, and WX performed immunostaining of HIE tissue with guidance from SF. M. Koontz generated hiPSC-derived astrocytes using the methods of Krencik et al. and Li et al. with guidance from EMU. M. Krawcyzk and YZ supplied unpublished human astrocyte RNA-seq data for master regulator analysis.

\section{COMPETING INTERESTS STATEMENT}


M. Kampmann has filed a patent application related to CRISPRi and CRISPRa screening (PCT/US15/40449) and serves on the Scientific Advisory Board of Engine Biosciences, Casma Therapeutics, and Cajal Neuroscience, and is an advisor to Modulo Bio and Recursion Therapeutics. None of the other authors declare competing interests.

\section{DATA AVAILABILITY STATEMENT}

Bulk RNA-seq data of hiPSC-derived astrocytes generated in this study shown in Extended Data Fig. 2 are available on the Gene Expression Omnibus (GEO) under accession code GSE182307. The raw single-cell RNA-seq data and UMI count matrices from the CROP-seq experiment are available on GEO under accession code GSE182308. Processed data from the CRISPRi screens and CROP-seq experiment can also be interactively explored on CRISPRbrain (https://www.crisprbrain.org/).

\section{CODE AVAILABILITY STATEMENT}

The full analysis pipeline (including code and processed data objects) used for master regulator analysis, analysis of CROP-seq data, and integration with previously published single-cell RNAseq datasets is available at https://kampmannlab.ucsf.edu/inflammatory-reactive-astrocyteanalysis. 


\section{METHODS}

\section{Human iPSC (hiPSC) culture}

Human iPSCs (male WTC11 background ${ }^{77}$ ) were cultured in Essential 8 (E8) Medium (ThermoFisher Scientific cat. no. A1517001) on BioLite Cell Culture Treated Dishes (ThermoFisher Scientific) coated with Growth Factor Reduced, Phenol Red-Free, LDEV-Free Matrigel Basement Membrane Matrix (Corning cat. no. 356231) diluted 1:100 in DMEM/F12 (ThermoFisher Scientific cat. no. 11330032). Essential 8 Medium was replaced daily. When hiPSC colonies demonstrated mature morphology, the hiPSCs were either clump passaged with EDTA for routine maintenance or dissociated to a near single-cell suspension with Accutase Cell Dissociation Reagent (ThermoFisher Scientific cat. no. A11105-01) for applications requiring cell counting. For clump passaging with EDTA, hiPSCs were washed with Dulbecco's phosphate buffered saline (DPBS; Milipore Sigma cat. no. D8537) and then incubated with Versene (ThermoFisher Scientific cat. no. 15040066) for 5-7 min at room temperature; the Versene solution was then aspirated and replaced with E8 + 10 nM Y-27632 dihydrochloride ROCK inhibitor (Tocris cat. no. 125410); hiPSC colonies were then gently detached mechanically using a cell scraper, resuspended gently, and passaged at 1:10-1:30 dilution in E8 + Y-27632, with Y27632 removed the next day. For near single-cell dissociation, hiPSCs were washed with DPBS, incubated with Accutase for $5-10 \mathrm{~min}$ at $37^{\circ} \mathrm{C}$, and then gently triturated with a $\mathrm{P} 1000$ pipette tip; the cell suspension was then diluted with PBS, collected into conical tubes, and spun down at $300 \mathrm{~g}$ for $3 \mathrm{~min}$; hiPSCs were then resuspended in $\mathrm{E} 8+\mathrm{Y}-27632$, counted, and plated onto Matrigel-coated plates at the desired density in E8 + Y-27632; Y-27632 would be maintained until the hiPSC colonies reached the appropriate size ( $>\sim 40$ cells). Studies with hiPSCs at UCSF were approved by the The Human Gamete, Embryo and Stem Cell Research (GESCR) Committee. Informed consent was obtained from the human subject when the WTC11 line ${ }^{77}$ was originally derived.

\section{Cloning of NFIA and SOX 9 cDNA into dox-inducible cassette}

To obtain NFIA cDNA, we designed PCR primers to amplify cDNA corresponding to transcript ENST00000403491(NFIA isoform 1) from astrocyte cDNA (Forward primer complementary sequence: ATGTATTCTCCGCTCTGTCTCAC; reverse primer complementary sequence: TCCCAGGTACCAGGACTGTG). We chose to amplify cDNA corresponding to NFIA isoform 1 because the cDNA clone (BC022264) used in Li et al. ${ }^{19}$ corresponds to transcript ENST00000371187 (NFIA isoform 2), which we found was not expressed highly in human astrocytes. To obtain SOX9 cDNA, we ordered cDNA clone OHu19789 (which corresponds to MGC clone BC056420) from GeneScript and then amplified SOX9 cDNA from the plasmid (Forward primer complementary seqeunce: ATGAATCTCCTGGACCCCTTCA; reverse primer complementary sequence: TCAAGGTCGAGTGAGCTGTGT). We then inserted NFIA and SOX9 cDNA joined by a T2A sequence into an AAVS1 safe-harbor plasmid containing a doxinducible cassette (Addgene plasmid no. 105840, gift from Michael Ward; digested with AflIII and ClaI) using Gibson assembly (New England Biolabs; cat. no. E2611L), resulting in pKL100.

\section{Generation of hiPSC line with stable integration of NFIA-SOX9 cassette and CRISPRi cassette}

WTC11 hiPSCs were transfected with pC13N-dCas9-BFP-KRAB ${ }^{78}$ (Addgene plasmid no. 127968) to stably integrate constitutive CRISPRi machinery into the CLYBL locus using 
TALEN-based editing as previously described ${ }^{78}$. CRISPRi WTC11 hiPSCs were then transfected with pKL100 to stably integrate the dox-inducible NFIA-SOX9 cassette into the AAVS1 locus using the same TALEN-based editing approach. Briefly, CRISPRi WTC11 hiPSCs were dissociated with Accutase to a single-cell suspension and plated at 500,000 cells per well in a Matrigel-coated 6-well plate in E8 $+10 \mu \mathrm{M}$ Y-27632. The next day, a media change with E8 + Y-27632 was performed and then the hiPSCs were transfected with $2.5 \mathrm{ug}$ of pKL100, $1.25 \mathrm{ug}$ of left and right AAVS1 TALEN plasmids (Addgene plasmid no. 59025 and 59026, gift from Danwei Huangfu), and 0.5 ug of Bcl-XL plasmid (pEF1-BCL-XL-wpre-polyA P1102, gift from Xiaobing Zhang, described in ref ${ }^{79}$ ) using Lipofectamine Stem Transfection Reagent (ThermoFisher Scientific cat. no. STEM00015) following the manufacture's protocol. After hiPSCs reached confluence, they were dissociated with Accutase and passaged to a $10 \mathrm{~cm}$ dish in $\mathrm{E} 8+10 \mathrm{uM} \mathrm{Y}-27632+0.1 \mathrm{ug} / \mathrm{mL}$ puromycin (ThermoFisher Scientific cat. no. A1113803) to select for clones with stable integration. Y-27632 was maintained until stable colonies formed, and puromycin was maintained for 5-7 days. The hiPSCs were then sorted for mCherry+ cells, which were then plated at 500,000 cells per well in a Matrigel-coated 6-well plate for transfection with $1.2 \mathrm{ug}$ of Cre recombinase mRNA (TriLink Biotechnologies cat. no. L-7211) using Lipofectamine Stem to remove the puromycin resistance gene and mCherry. The Cretransfected hiPSCs were then expanded and sorted for mCherry- cells, which were then plated for colony picking to generate monoclonal hiPSC lines. hiPSC clones were tested for integration of the NFIA-SOX9 cassette and removal of the puromycin resistance and mCherry by genomic PCR with the following pairs of primers:

AAVS1 FWD: CTGCCGTCTCTCTCCTGAGT bGHpolyA REV: GCTGGCAACTAGAAGGCACAG

\section{AAVS1 FWD: CTGCCGTCTCTCTCCTGAGT Puro REV: GTGGGCTTGTACTCGGTCAT \\ TRE3G FWD: GTGTTGTGGAATTGCTCCAG AAVS1 REV: AAGAGTGAGTTTGCCAAGCAGT}

\section{Neural induction of hiPSCs}

Embryoid body (EB)-based neural induction of WTC11 hiPSCs stably integrated with CRISPRi machinery and dox-inducible NFIA-SOX9 was performed as previously described ${ }^{80}$ with some modifications. Briefly, on day 0, hiPSCs were dissociated to a near single-cell suspension with Accutase, resuspended in neural induction media (NIM; see recipe below) + 10 nM Y-27632, and then transferred to an Aggrewell 800 plate (StemCell Technologies cat. no. 34815) precoated with Anti-adherence rinsing solution (StemCell Technologies cat. no. 07010) at 3 million cells per well for EB formation. The next day (day 1), a half media change with NIM was performed and LDN193189 (LDN; Tocris cat. no. 6053) and SB431542 (SB; Tocris cat. no. 1614) were added to final concentrations of $0.1 \mu \mathrm{M}$ and $10 \mu \mathrm{M}$, respectively. A half media change of NIM + LDN + SB was performed every day until day 7, when EBs were transferred to a Matrigel-coated 6-well plate after performing a half media change (one well from the Aggrewell plate would be transferred to one well in the 6-well plate). The next day (day 8), a full 
media change with NIM + LDN + SB was performed, and then every other day afterwards until day 14, during which time neural rosettes would appear in the attached EBs. On day 14, neural rosettes were detached non-enzymatically with the following method: the attached EBs were incubated Neural Rosette Selection Reagent (NRSR; StemCell Technologies cat. no. 05832) for 1 hour at $37{ }^{\circ} \mathrm{C}$ to weaken the attachment of the neural rosettes, the NRSR was aspirated and replaced with DMEM/F12, and the rosettes were detached with targeted jetting of DMEM/F12 using a wide-orfice P1000 pipette tip and then collected into a conical tube; targeted jetting with DMEM/F12 and collection of released rosettes was repeated until the majority of rosettes had detached. Rosettes were then spun down at $100 \mathrm{~g}$ for $3 \mathrm{~min}$, resuspended in NPC media (see recipe below), and transferred to a Matrigel-coated 6-well plate (rosettes collected from 1-2 wells would be replated into 1 well depending on the yield). From day 15 to 21 , a full media change with NPC media was performed every other day, during which time neural progenitor cells (NPCs) would spread out from the attached rosettes and cover the well completely. Once NPCs reached confluency, they were dissociated with Accutase and replated at high density (at least 1 million cells per well of a 6-well plate) in Matrigel-coated plates for expansion.

Neural induction media (NIM) formulation:

DMEM/F12 (+HEPES, +Glutamine) basal media (ThermoFisher Scientific cat. no. 11330032)

$2 \%$ (v/v) B27 supplement minus Vit. A (ThermoFisher Scientific cat. no. 12587010)

$1 \%$ (v/v) N2 supplement (ThermoFisher Scientific cat. no. 17502048)

Neural progenitor cell (NPC) media formulation:

DMEM/F12 (+HEPES, +Glutamine) basal media (ThermoFisher Scientific cat. no. 11330032)

$2 \%$ (v/v) B27 supplement minus Vit. A (ThermoFisher Scientific cat. no. 12587010)

$1 \%$ (v/v) N2 supplement (ThermoFisher Scientific cat. no. 17502048)

20 ng/mL bFGF (PeproTech cat. no. 100-18B)

\section{Purification of NPCs}

To remove contaminating neural crest cells from the NPC cultures, we used fluorescence activated cell sorting (FACS) to select for CD133+/CD271- cells following the protocol described in Cheng et al. ${ }^{80}$ with modifications. Briefly, NPCs were dissociated to a single-cell suspension with Accutase, resuspended in FACS buffer (see recipe below), and then incubated with PE-conjugated CD133 antibody (1:50 dilution; Miltenyi Biotec cat. no. 130-113-108) and PerCP-Cy5.5-conjugated CD271 antibody (1:50 dilution; BD Biosciences cat. no. 560834), including single antibody-stained and unstained controls. After antibody incubation, the cell suspension was diluted 10x with FACS buffer, spun down, and resuspended in FACS buffer for sorting. CD133+/CD271 - cells were sorted using a BD FACSAria Fusion cell sorter at 5,00010,000 events per second, and then plated at 100,000 cells per $\mathrm{cm}^{2}$ onto Matrigel-coated plates. Media was then changed every other day until the NPCs reached confluency, at which point the NPCs were passaged for expansion. After 2-3 additional expansion passages after sorting, NPCs were characterized by immunostaining and qPCR for NPC markers such as PAX6 and Nestin, and then cryopreserved in NPC media $+10 \%$ DMSO.

FACS buffer formulation:

DPBS (Milipore Sigma cat. no. D8537) 
$1 \%$ (w/v) BSA (Milipore Sigma cat. no. A9647)

2 mM EDTA (Milipore Sigma cat. no. 324506)

\section{Generation of iAstrocytes from NPCs}

For iAstrocyte differentiation, CD133+/CD271- sorted NPCs generated from WTC11 iPCS with stably integrated CRISPRi machinery and dox-inducible NFIA-SOX9 were dissociated to a single-cell suspension with Accutase and then replated at 7,500 cells per $\mathrm{cm}^{2}$ in NPC media onto a 10-cm or 15-cm dish coated with Matrigel diluted at 1:200 in DMEM/F12. The next day, media was changed to ScienCell Astrocyte Media (ScienCell Research Laboratories cat. no. $1801)+2 \mu \mathrm{g} / \mathrm{mL}$ doxycycline (Millipore Sigma cat. no. D9891) to initiate iAstrocyte differentiation. A full media was then changed every other day, with doxycycline maintained at 2 $\mu \mathrm{g} / \mathrm{mL}$ throughout the differentiation process. When the differentiating NPCs reached confluency within 3-4 days, the culture was dissociated with Accutase and split 1:10 onto new Matrigel coated dishes for expansion, with some cells saved for cryopreservation. After confluency was reached within 5-6 days, the cultures were dissociated and split 1:8, saving some cells for cryopreservation. Expansion of the cultures at 1:8 split with cryopreservation of cells after each split was continued until day 20 of differentiation, yielding iAstrocytes.

\section{Generation of hiPSC-derived astrocytes using alternative protocols}

In parallel to the generation of iAstrocytes, hiPSC-derived astrocytes were also generated from CD133+/CD271- sorted NPCs according to the protocol published in TCW et al. ${ }^{18}$, which mirrored the process described above for iAstrocyte generation with the exception of adding doxycycline. In addition to the protocol published in TCW et al., hiPSC-derived astrocytes were also generated from WTC11 iPSCs with stably integrated CRISPRi machinery and dox-inducible NFIA-SOX9 according to Li et al. ${ }^{19}$, or from WTC11 iPSCs with stably integrated CRISPRi machinery according to Krencik et $a l .{ }^{15}$.

\section{Induction of inflammatory reactivity in hiPSC-derived astrocytes}

iAstrocytes or hiPSC-derived astrocytes generated according to TCW et al. ${ }^{18}$ were plated onto Matrigel-coated (1:200 diluted Matrigel) 96-well plates, 24-well plates or 6-well plates at 20,000 cells per $\mathrm{cm}^{2}$ in ScienCell Astrocyte Media without addition of doxycycline; hiPSC-derived astrocytes generated according to Li et al. ${ }^{19}$ or Krencik et al. ${ }^{15}$ were plated onto Matrigel-coated (1:200 diluted Matrigel) 96-well plates at $\sim 62,500$ cells per $\mathrm{cm}^{2}$ in Astrocyte Maturation Media (AMM; see recipe below) without addition of doxycycline. A full media change was performed the next day, and then every other day afterwards. Five days after plating, hiPSC-derived astrocytes were treated with vehicle control or IL-1 $\alpha$ (3 ng/mL; Peprotech cat. no. AF-200-01A), TNF (30 ng/mL; Peprotech cat. no. AF-300-01A), and C1q (400 ng/mL; Complement Technology cat. no. A099) with a full media change in the appropriate media to induce inflammatory reactivity according to Liddelow et al. ${ }^{8}$. All assays were performed at 24 hours after cytokine treatment (see Fig. 1g) unless otherwise stated.

Astrocyte Maturation Media (AMM) formulation:

DMEM/F12 (+HEPES, +Glutamine) basal media (ThermoFisher Scientific cat. no. 11330032) $1 \%$ (v/v) B27 supplement minus Vit. A (ThermoFisher Scientific cat. no. 12587010) $0.5 \%(\mathrm{v} / \mathrm{v}) \mathrm{N} 2$ supplement (ThermoFisher Scientific cat. no. 17502048) 
$1 \%$ (v/v) Antibiotic-Antimycotic (ThermoFisher Scientific cat. no. 15240062)

$10 \mathrm{ng} / \mathrm{mL}$ CNTF (R\&D Systems cat. no. 257-NT)

10 ng/mL BMP-4 (R\&D Systems cat. no. 314-BP)

\section{CRISPRi-mediated gene knockdown in hiPSC-derived astrocytes using lentiviral sgRNA delivery}

For experiments involving CRISPRi-mediated gene knockdown, iAstrocytes were transduced with lentivirus containing single-guide RNAs (sgRNAs) at the time of plating (see Fig. 2b). CRISPRi sgRNAs were cloned into pMK1334 ${ }^{78}$ (Addgene cat. no. 127965) digested with BstXI and BlpI as previously described in Gilbert et al. ${ }^{81}$. Lentivirus containing sgRNAs was produced by transfecting HEK293T cells with pMK1334 and $3^{\text {rd }}$ generation lentiviral packaging plasmids with TransIT-Lenti Transfection Reagent (Mirus cat. no. MIR6606) according to the manufacturer's instructions. The lentivirus was then precipitated using Lentivirus Precipitation Solution (ALSTEM cat. no. VC150) according to the manufacturer's instructions, resuspended in DPBS at $1 / 10$ of the original volume, and then aliquoted and stored at $-80{ }^{\circ} \mathrm{C}$. The functional titer of the lentivirus was then tested on iAstrocytes by serial dilution followed by measurement of BFP+ cells 48 hours after transduction. For all experiments involving CRISPRi-mediated gene knockdown in iAstrocytes, sufficient sgRNA lentivirus was added to transduced $>70 \%$ of iAstrocytes.

\section{Immunostaining of astrocyte markers}

iAstrocytes and hiPSC-derived astrocytes generated according to TCW et al. ${ }^{18}$ were plated at 20,000 cells per $\mathrm{cm}^{2}$ onto a Greiner $\mu$ Clear 96-well plate (Greiner Bio-One cat. no. 655087) coated with 1:200 diluted Matrigel, and then treated with vehicle control or IL-1 $\alpha+\mathrm{TNF}+\mathrm{C} 1 \mathrm{q}$ as described above. 24 hours after cytokine treatment, the astrocytes were washed with DPBS and then fixed with $4 \%$ paraformaldehyde (diluted from a 16\% solution; Electron Microscopy Sciences cat. no. 15710) for $15 \mathrm{~min}$ at room temperature (RT). After washing three times with DPBS, blocking and permeabilization was performed with DPBS $+3 \% \mathrm{BSA}+0.1 \%$ Triton X100 (Millipore Sigma cat. no. X100) for $30 \mathrm{~min}$ at RT. Primary antibodies against GFAP (1:500, rabbit polyclonal; ThermoFisher Scientific cat. no. PA1-10019), S100ß (1:500, mouse monoclonal; Millipore Sigma cat. no. S2532), GLAST (1:500, mouse monoclonal; Miltenyi Biotec cat. no. 130-095-822), or NFIA (1:200 rabbit polyclonal; Atlas Antibodies cat. no. HPA008884) were then added in blocking buffer and incubated overnight at $4{ }^{\circ} \mathrm{C}$. Afterwards, the samples were washed with DPBS $+0.1 \%$ Triton X-100 three times, incubated with preadsorbed secondary antibodies (1:500 Goat anti-mouse IgG AF647, 1:500 Goat anti-rabbit IgG AF555; Abcam cat. no. ab150119 and ab150086) for 1 hour at RT, washed two times with DPBS $+0.1 \%$ Triton X-100, incubated with $1 \mu \mathrm{g} / \mathrm{mL}$ Hoechst (ThermoFisher Scientific cat. no. H3570) and 1:10 ActinGreen 488 (ThermoFisher Scientific cat. no. R37110) for 20 min at RT, and then washed two additional times before imaging on an IN Cell Analyzer 6000, using a 20X 0.45 NA objective, 2x2 binning, 100-400 ms exposure, an aperture width of $\sim 1$ Airy unit, and 16 fields per well.

\section{Generation of hiPSC-derived brain endothelial-like cells and measurement of barrier integrity}


hiPSCs were differentiated to brain endothelial-like cells as previously described ${ }^{82}$. Briefly, hiPSCs were dissociated with Accutase and seeded on Matrigel-coated plates in E8 medium containing $10 \mu \mathrm{M}$ Y 27632 at a density of $15,000 \mathrm{cells} / \mathrm{cm}^{2}$. Differentiation was initiated 24 hours after seeding by changing to E6 medium, with daily medium changes for 4 days. Next, cells were expanded with serum-free basal endothelial cell medium (EC medium) supplemented with 50x diluted B27 (Thermo Fisher Scientific), 1x GlutaMAX (Thermo Fisher Scientific), $10 \mu \mathrm{M}$ retinoic acid (Sigma Aldrich), and $20 \mu \mathrm{g} / \mathrm{ml}$ FGF2 for 2 days without a media change. Following this treatment, cells were collected by a 20-minute incubation in Accutase and seeded onto Transwell filters $\left(1.1 \mathrm{~cm}^{2}\right.$ polyethylene terephthalate membranes with $0.4 \mu \mathrm{m}$ pores; Fisher Scientific) coated with a mixture of $400 \mu \mathrm{g} / \mathrm{ml}$ collagen IV (Sigma Aldrich) and $100 \mu \mathrm{g} / \mathrm{ml}$ fibronectin (Sigma Aldrich). The following day, cells were switched to EC medium lacking FGF2 and RA. For co-culture with iAstrocytes, filters were transferred to 12-well plates containing iAstrocytes and the same medium was utilized. Starting at this time, transendothelial electrical resistance (TEER) was measured using STX2 chopstick electrodes and an EVOM2 voltameter (World precision Instruments) approximately every 24 hours. TEER readings on empty Transwell filteres were subtracted from all measurements to reflect the resistance of only the cultured cells.

\section{Generation of hiPSC-derived neurons (iNeurons) and GCaMP iNeurons}

hiPSC-derived neurons (iNeurons) were generated from WTC11 hiPSCs with stably integrated dox-inducible NGN2 (NGN2 iPSCs) according to Fernandopulle et al. ${ }^{83}$. To generate GCaMP iNeurons, NGN2 iPSCs were transduced with a lentivirus delivering GCaMP6m (gift from Dr. Michael Ward). To facilitate segmentation of neuron soma, an additional lentivirus transduction was performed to deliver pMK1334 containing a non-targeting sgRNA, which confers BFP expression localized to the nucleus. Clonal lines were then isolated by colony picking, differentiated to neurons according to Fernandopulle et $a l^{83}$, and evaluated for homogeneity of GCaMP6m expression and the presence of spontaneous calcium oscillations. A clonal line satisfying the above criteria was selected for GCaMP imaging experiments.

\section{Measurement of calcium activity in GCaMP iNeurons}

Briefly, GCaMP iNeurons at day 3 of differentiation (see Fernandopulle et al. ${ }^{83}$ ) were replated onto poly-D-lysine-coated 96-well plates (Corning cat. no. 354640) at 62,5000 cells per $\mathrm{cm}^{2}$ in neuron media $+2 \mu \mathrm{g} / \mathrm{mL}$ doxycycline. For co-culture experiments, iAstrocytes were added on day 3 at 10,000 cells per $\mathrm{cm}^{2}$ in an equivalent volume of ScienCell Astrocyte Media $+2 \mu \mathrm{g} / \mathrm{mL}$ doxycycline. For mono-culture experiments, an equivalent volume of neuron media $+2 \mu \mathrm{g} / \mathrm{mL}$ doxycycline was added. On day 6 , half of the media was replaced with fresh neuron media +2 $\mu \mathrm{g} / \mathrm{mL}$ doxycycline; on day 10 , half of the media was replaced with fresh neuron media without doxycycline. On day 17 , the cultures were treated with vehicle control or IL-1 $\alpha+\mathrm{TNF}+\mathrm{C} 1 \mathrm{q}$ (using the same final concentration as described above for astrocyte experiments) by performing a half media change with fresh neuron media. Calcium activity in GCaMP iNeurons was recorded on day 18 and day 20 with an IN Cell Analyzer 6000, using a 20X 0.45 NA objective, $2 \times 2$ binning, environmental control set to $37{ }^{\circ} \mathrm{C}$ and $5 \% \mathrm{CO}_{2}$, an aperture width of $\sim 1$ Airy unit, and 1 frame ( $800 \mathrm{~ms}$ exposure) collected per second for 40 seconds per field ( 1 field per well).

\section{Isolation of synaptosomes and labeling with pHrodo}


Synaptosomes were isolated from fresh Innovative Grade US Origin Rat Sprague Dawley Brains (Innovative Research, Inc.; Cat. No. IGRTSDBR) with the Syn-PERTM Synaptic Protein Extraction Reagent (ThermoFisher Scientific cat. no. 87793) according to the manufacture's protocol with minor changes. Briefly, $10 \mathrm{~mL}$ of Syn-PER Reagent supplemented with 1x protease inhibitor cOmplete Mini, EDTA free (Roche cat. no. 11836170001) and 1x phosphatase inhibitor PhosSTOP (Roche cat. no. 4906845001) were added per gram of brain tissue. Dounce homogenization was performed on ice and homogenate was transferred to a conical tube and centrifuged at $1200 \times \mathrm{g}$ for 10 minutes at $4^{\circ} \mathrm{C}$. The pellet was discarded, the supernatant was transferred to a new tube, and the centrifugation step was repeated. The supernatant was then centrifuged at $15,000 \times \mathrm{g}$ for 20 minutes at $4{ }^{\circ} \mathrm{C}$. The supernatant was removed and the wet pellet was weighed. The synaptosome fractions were resuspended at a concentration of $50 \mathrm{mg} / \mathrm{ml} .3$ $\mu \mathrm{M}$ of pHrodo Red, succinimidyl ester (ThermoFisher Scientific cat. no. P36600) was added to the synaptosome fraction and incubated for $45 \mathrm{~min}$ at room temperature in the dark. After diluting the solution 1:10 in DPBS, the synaptosomes were spun down at $2500 \times \mathrm{g}$ for $5 \mathrm{~min}$. The supernatant was removed and then the synaptosomes were washed two times with DPBS. The pHrodo-labelled synaptosomes were resuspended in DMEM/F12 + 5\% DMSO at a stock concentration of $50 \mathrm{mg} / \mathrm{ml}$, aliquoted, and then frozen in liquid nitrogen for later use.

\section{Measurement of synaptosome phagocytosis}

For synaptosome phagocytosis experiments, pHrodo-labeled rat synaptosomes were used for iAstrocytes, and pHrodo-labeled iNeuron synaptosomes were used for Li et al. ${ }^{19}$ and Krencik et $a l .{ }^{15}$ hiPSC-derived astrocytes. Briefly, astrocytes were incubated with pHrodo-labeled synaptosomes resuspended in the appropriate astrocyte media (ScienCell Astrocyte media for iAstrocytes, AMM for Li et al. and Krencik et al. astrocytes) at $1 \mathrm{mg} / \mathrm{mL}$ for 3 hours at $37{ }^{\circ} \mathrm{C}$; for negative controls, some samples were pre-treated with $10 \mathrm{uM}$ cytochalasin D (Millipore Sigma cat. no. C8273) for $15 \mathrm{~min}$ and also incubated with synaptosomes in the presence of $10 \mathrm{uM}$ cytochalasin D to inhibit phagocytosis. After incubation with pHrodo-labeled synaptosomes, astrocytes were washed with DPBS, dissociated with Accutase, and pHrodo fluorescence was measured by flow cytometry. The gating strategy to determine the percent of phagocytic cells was based on the separation between the fluorescence histograms of samples treated or not treated with cytochalasin D.

\section{Measurement of iNeuron viability in the presence of astrocyte conditioned media}

Conditioned media was collected from iAstrocytes treated with vehicle control or IL$1 \alpha+\mathrm{TNF}+\mathrm{C} 1 \mathrm{q}$ for 24 hours, spun down at $300 \mathrm{~g}$ for $10 \mathrm{~min}$ to remove dead cells, and transferred to day 17 iNeurons after removing the original iNeuron media. Unconditioned ScienCell Astrocyte Media was used as a negative control. After 72 hours, iNeuron viability was assessed by adding $10 \mathrm{ug} / \mathrm{mL}$ Hoechst and $1 \mu \mathrm{M}$ TO-PRO-3 (ThermoFisher Scientific cat. no. T3605) in DPBS, incubating for $10 \mathrm{~min}$ at $37^{\circ} \mathrm{C}$, and then imaging on an IN Cell Analyzer 6000, using a 10X 0.45 NA objective, $2 \times 2$ binning, environmental control set to $37{ }^{\circ} \mathrm{C}$ and $5 \% \mathrm{CO}_{2}$, an aperture width of $\sim 1$ Airy unit, $200 \mathrm{~ms}$ exposure, and 4-9 fields per well. The percent of dead neurons (stained by TO-PRO-3) was calculated after image processing and segmentation with CellProfiler (see Data Analysis section).

\section{Bulk RNA-seq library prep}


hiPSC-derived astrocytes (iAstrocytes, TCW et al., Li et al., and Krencik et al. astrocytes), NPCs, and iNeurons were cultured in their respective media and treated with vehicle control or IL-1 $\alpha+\mathrm{TNF}+\mathrm{C} 1 \mathrm{q}$ for 24 hours. RNA extraction was then performed with Zymo Quick-RNA Microprep kit (Zymo Research cat. no. R1051). 50-100 ng of RNA was then used to construct bulk RNA-seq libraries using the QuantSeq 3' mRNA-Seq Library Prep Kit FWD for Illumina (Lexogen cat. no. 015.96) following the manufacturer's instructions. The concentration of QuantSeq libraries mRNA-seq library was quantified using the Qubit dsDNA HS Assay Kit (ThermoFisher Scientific cat. no. Q32851) on a Qubit 2.0 Fluorometer. Library fragment-length distributions were quantified with High Sensitivity D5000 Reagents (Agilent Technologies cat. no. 5067-5593) on the 4200 TapeStation System. The libraries were sequenced on an Illumina NextSeq 2000 instrument with single-end reads.

\section{Antibody staining for flow cytometry}

For antibody staining of cell-surface proteins (VCAM1, TFRC), hiPSC-derived or primary mouse astrocytes were dissociated with Accutase, washed with DPBS, incubated with conjugated primary antibodies for 20 min on ice in DPBS $+1 \%$ BSA, washed with DPBS, and then resuspended in DPBS $+1 \%$ BSA for flow cytometry. For antibody staining of intracellular proteins (C3), hiPSC-derived astrocytes were dissociated with Accutase, washed with DPBS, fixed with $2 \%$ paraformaldehyde for $10 \mathrm{~min}$ at RT, washed twice with DPBS $+0.5 \%$ Tween 20 (Millipore Sigma cat. no. P9461), incubated with unconjugated primary antibody for 20 min at RT, washed with DPBS $+0.5 \%$ Tween 20, incubated with conjugated secondary antibody for 20 min at RT, washed with DPBS $+0.5 \%$ Tween 20, and then resuspended in DPBS $+0.5 \%$ Tween 20 for flow cytometry. For dual staining of VCAM1 and C3, the protocol for intracellular protein staining was used.

Conjugated primary antibodies:

PE-Cy7 mouse anti-VCAM1 (1:80 dilution; BioLegend cat. no. 305818)

PE-Cy7 mouse anti-TFRC (1:80 dilution; BioLegend cat. no. 334112)

Unconjugated primary antibodies:

Mouse anti-C3 (1:250 dilution; BioLegend cat. no. 846302)

Rabbit anti-VCAM1 (1:250 dilution; Abcam cat. no. ab134047)

Conjugated secondary antibodies:

AF488 goat anti-mouse IgG (1:1000 dilution; ThermoFisher Scientific cat. no. A-11029)

AF568 goat anti-rabbit IgG (1:1000 dilution; ThermoFisher Scientific cat. no. A-11036)

\section{Pooled CRISPRi screening}

To identify transcriptional regulators of inflammatory reactivity, we created a custom sgRNA library targeting the human transcription factors ${ }^{36}$, using $5 \mathrm{sgRNAs}$ with the highest predicted activity scores from Horlbeck et al. ${ }^{39}$ per gene. The library was created by cloning a pool of sgRNA-containing oligonucleotides custom-synthesized by Agilent Technologies into our optimized sgRNA expression vector as previously described ${ }^{81}$. To screen against the druggable genome, we used the $\mathrm{H} 1$ sub-library from Horlbeck et al. ${ }^{39}$. The transcription factor and druggable genome libraries were packaged into lentivirus as previously described ${ }^{78}$. For each 
experimental replicate, $\sim 10$ million iAstrocytes were plated onto 4 Matrigel-coated 15-cm dishes, transduced with the lentiviral transcription factor or H1 sgRNA library at high multiplicity of infection so that $>70 \%$ of cells were transduced, treated with vehicle control or IL- $1 \alpha+\mathrm{TNF}+\mathrm{C} 1 \mathrm{q}$ for 24 hours, and then incubated with pHrodo-labeled rat synaptosomes or stained for cell-surface VCAM1 (using the PE-Cy7 mouse anti-VCAM1 antibody) as shown in Fig. 3a. iAstrocytes were sorted into pHrodo high vs. low or VCAM1 high vs. low (top and bottom $30 \%$ of cells) using a BD FACSAria Fusion cell sorter at 5,000-10,000 events per second, and then pelleted for genomic DNA extraction. sgRNA abundances were then measured using next-generation sequencing as previously described ${ }^{78}$. The screens were performed with two experimental replicates per condition.

\section{CROP-seq}

sgRNAs targeting the top hits from the CRISPRi screens and also candidate regulators selected based on literature were cloned into pMK1334. The concentration of each sgRNA plasmid was measured using the Qubit dsDNA HS Assay Kit on a Qubit 2.0 Fluorometer, and then the plasmids were pooled. iAstrocytes were transduced with lentivirus generated from the sgRNA pool at low multiplicity of infection so that $<30 \%$ of cells were transduced, treated with vehicle control or IL-1 $\alpha+\mathrm{TNF}+\mathrm{C} 1 \mathrm{q}$ for 24 hours, and then sorted for sgRNA-transduced cells via FACS. Sorted iAstrocytes were then used as input for single-cell RNA-seq using Chromium Next GEM Single Cell 3' v3.1 reagents (10X Genomics cat. no. PN-1000121, PN-1000127, and PN1000213), loading 45,000 cells per reaction into four reactions, with two reactions for vehicle control-treated iAstrocytes and two reactions for IL-1 $\alpha+\mathrm{TNF}+\mathrm{C} 1 \mathrm{q}$-treated iAstrocytes. To facilitate association of single-cell transcriptomes with sgRNAs, sgRNA-containing transcripts were amplified as described in Tian et al. ${ }^{78}$. The sgRNA-enrichment libraries were separately indexed and sequenced as spike-ins alongside the whole-transcriptome single-cell RNA-seq libraries on a NovaSeq 6000 , recovering on average $\sim 29,000$ transcriptome reads and $\sim 5,000$ sgRNA-containing transcript reads per cell.

\section{Measurement of cytokine concentrations}

Conditioned media was collected from iAstrocytes, spun down at $300 \mathrm{~g}$ for $10 \mathrm{~min}$ to remove debris, and then frozen at $-80{ }^{\circ} \mathrm{C}$ until analysis. The concentration of selected cytokines (IFN$\alpha 2 \mathrm{a}$, IFN- $\beta$, IFN- $\gamma$, IL-6, CXCL10, GM-CSF) was measured by multi-array electrochemiluminescence using custom U-PLEX plates from Meso Scale Discovery following the manufacturer's instructions.

\section{Immunostaining of human neuropathological samples}

\section{Alzheimer's disease \\ Brain tissue collection procedures were approved by the Institutional Review Board at Vanderbilt University Medical Center, and written consent for brain donation was obtained from patients or their surrogate decision makers. A diagnostic post-mortem evaluation was performed to confirm the presence of Alzheimer's disease following the National Alzheimer's Coordinating Center (NACC) Neuropathology Data Form. Tissue for this study was flash-frozen in liquid nitrogen at the time of donation. Cryosectioned tissues were fixed with $4 \%$ paraformaldehyde, photobleached with a broad-spectrum LED array for two days, and then processed for}


immunofluorescence staining. Samples were permeabilized with $0.3 \%$ Triton X-100 in PBS, blocked with $10 \%$ goat serum in PBS, and incubated at $4 \square$ overnight with the following primary antibodies: mouse anti-C3 (1:200 dilution; Biolegend cat. no. 846302), chicken anti-GFAP (1:300 dilution; Aves Labs cat. no. GFAP), rabbit anti-VCAM1 (1:200 dilution; Abcam cat. no. ab134047). The following day, after washing with PBS, immunostaining was completed by a 1 hour room temperature incubation with secondary antibodies (goat anti-rabbit Alexa Fluor 488, goat anti-mouse Alexa Fluor 546, and goat anti-chicken Alexa Fluor 647; 1:1,000 dilution; ThermoFisher Scientific). After additional washes, tissue sections were mounted with the antifade Fluoromount-G medium containing DAPI (Southern Biotechnology). Images were acquired with a Leica DMi8 epifluorescence microscope. 6-8 fields were collected per sample.

\section{Hypoxic ischemic encephalopathy}

All human HIE tissue was collected with informed consent and in accordance with guidelines established by UCSF Committee on Human Research (H11170-19113-07) as previously described $^{84}$. Immediately after procurement, all brains were immersed in PBS with $4 \%$ paraformaldehyde for $3 \mathrm{~d}$. On day 3 , the brain was cut in the coronal plane at the level of the mammillary body and immersed in fresh $4 \%$ paraformaldehyde and PBS for an additional $3 \mathrm{~d}$. After fixation, all tissue samples were equilibrated in PBS with 30\% sucrose for at least $2 \mathrm{~d}$. After sucrose equilibration, tissue was placed into molds and embedded with optimal cutting temperature medium for $30 \mathrm{~min}$ at room temperature followed by freezing in dry ice-chilled ethanol. UCSF neuropathology staff performed brain dissection and its evaluation. The diagnosis of HIE requires clinical and pathological correlation; no widely accepted diagnostic criteria are present for the pathological diagnosis of HIE. HIE cases showed consistent evidence of diffuse white matter gliosis, as evaluated by the qualitative increase in the number of GFAP-positive cells in addition to the increased intensity of GFAP staining. For immunostaining of reactive astrocyte markers, tissue slides were bleached with UV overnight, rinsed with PBS for 10 minutes, incubated with blocking solution (10\% normal goat serum $+0.2 \%$ Triton $X-100$ in PBS) for 1 hour at room temperature, and then incubated with the following primary antibodies overnight at $4 \square$ : mouse anti-C3 (1:200 dilution; Biolegend cat. no. 846302) + rabbit anti-GFAP (1:500 dilution; Agilent Dako cat. no. Z0334), or mouse anti-GFAP (1:500 dilution; Milipore Sigma cat. no. G3893) + rabbit anti-VCAM1 (1:200 dilution; Abcam cat. no. ab134047). Afterwards, the samples were rinsed three times with PBS $+0.2 \%$ Triton X-100 for 10 minutes each time, and then incubated with secondary antibodies for 1 hour at room temperature in the dark.

\section{Data analysis}

\section{Pooled CRISPRi screens}

We analyzed the data from the pooled CRISPRi screens using the MAGeCK-iNC bioinformatic pipeline previously described in Tian et al. ${ }^{78}$.

\section{Master regulator analysis (MRA)}

To collect human astrocyte RNA-seq data for co-expression network reconstruction, we downloaded gene-level counts from samples annotated as "Astrocyte" in ARCHS4 ${ }^{85}$. For published human astrocyte RNA-seq datasets not found in ARCHS4, we manually downloaded 
FASTQ files from GEO, and then processed the FASTQ data using Elysium ${ }^{86}$, which implements the alignment pipeline used in ARCHS4. In addition to published human astrocyte RNA-seq datasets, we also used Elysium to process additional human astrocyte RNA-seq datasets from our labs (Ye Zhang lab, UCLA ${ }^{35}$, and Kampmann lab; all datasets used for MRA available at https://kampmannlab.ucsf.edu/inflammatory-reactive-astrocyte-analysis). We merged the gene-level count matrices from the above sources into a single matrix, which was then used for MRA (see Supplementary Table 11 for the metadata corresponding to all samples used for co-expression network reconstruction). Batch correction was first performed across datasets using ComBat-seq ${ }^{87}$, which is a part of the $\mathrm{R}$ (version 4.0.3) package "sva" (version 3.38.0). Gene-level counts were then transformed to log-scale using the variance-stabilizing transformation in DESeq2 (version 1.30.1). A list of human transcription factors (TFs) was obtained from Lambert et $a l .{ }^{36}$, and a list of human kinases and phosphatases was obtained from Manning et al. ${ }^{37}$ and Liberti et al. ${ }^{38}$. Genes and regulators (TFs, kinases and phosphatases) with low expression in hiPSC-derived astrocytes used in our study (mean transcripts per million $<1$ ) were removed from the matrix. MRA was then performed using the R package "RTN" (version 2.16.0) $)^{32-34}$, following the RTN vignette available on Bioconductor.

\section{CROP-seq}

Alignment and quantification were performed on 10X single-cell RNA-seq libraries and sgRNAenriched libraries using Cell Ranger (version 5.0.1) with default parameters and reference genome GRCh38-3.0.0. Cellranger aggr was used to aggregate counts belonging to the same sample across different GEM wells. sgRNA unique molecular identifier (UMI) counts for each cell barcode were obtained using the pipeline described in Hill et al. ${ }^{88}$. sgRNAs were assigned to cells using demuxEM ${ }^{89}$. The gene vs. cell barcode matrix outputted by Cell Ranger was converted into a SingleCellExperiment (SCE) object using the read10xCounts function from the DropletUtils R package (version 1.10.388). sgRNA assignments were appended to the SCE metadata and filtered to only include cells with a single sgRNA. The SCE object was then converted into a Seurat object for subsequent analysis. Data normalization, log-transformation, and identification of highly variable genes were performed using Seurat::SCTransform ${ }^{90}$.

For exploratory analysis of the effect of knocking down each regulator targeted in the CROP-seq experiment, a separate Seurat object was created for each regulator consisting of iAstrocytes transduced with a non-targeting (NTC) sgRNA and iAstrocytes transduced with the sgRNA targeting that regulator. For each of these Seurat objects, the data was renormalized with SCTransform, dimensionality reduction was performed with Seurat::RunPCA (30 PC's retained) and Seurat::RunUMAP, and clustering was performed with Seurat::FindNeighbors and Seurat::FindClusters (resolution $=0.5$ ). Regulators whose knockdown resulted in clear spatial separation between knockdown iAstrocytes and NTC iAstrocytes in UMAP were selected for further analysis. The PCA embeddings from the Seurat objects corresponding to the selected regulators were used for aligned $\mathrm{UMAP}^{91}$, which allows the effect of knocking down different regulators to be visualized in the same UMAP embedding. This aligned UMAP embedding was used for Fig. 4a,b,d and Fig. 5c, which displayed only NTC iAstrocytes. Markers of IRAS1 and IRAS2 in NTC iAstrocytes were identified by performing Student's t-tests on the Pearson residuals from SCTransform using Seurat::FindMarkers. 
To find genes whose differential expression induced by IL-1 $\alpha+\mathrm{TNF}+\mathrm{C} 1 \mathrm{q}$ treatment was changed by regulator knockdown, we used the R package limma (version 3.46.0) to perform linear regression on the Pearson residuals from running SCTransform on the Seurat object containing all iAstrocytes assigned with a single sgRNA. We used the design formula y regulatorKnockdown + cytokineTreatment + regulatorKnockdown:cytokineTreatment, where the interaction term regulatorKnockdown:cytokineTreatment reflects the effect of knocking down a given regulator on the differential expression induced by $\mathrm{IL}-1 \alpha+\mathrm{TNF}+\mathrm{C} 1 \mathrm{q}$ treatment. For each regulator knockdown, genes with a statistically significant interaction term were extracted for the analysis presented in Extended Data Fig. 4d-e.

To construct the heatmap in Extended Data Fig. 4e, the $\log _{2}$-fold-change (LFC) values of the above DEGs were weighted by their associated multiple testing-corrected $P$-values $\left(P_{\text {adj }}\right)$ to generate a gene score, where gene score $=\mathrm{LFC} *\left(1-P_{\text {adj }}\right)$. Hierarchical clustering (Ward's method) was then performed on both the rows (genes) and columns (regulators) of the gene score matrix, using Pearson correlation as the distance metric. The heatmap was drawn using $\mathrm{R}$ package ComplexHeatmap (version 2.6.2) ${ }^{92}$.

\section{Integration of iAstrocyte single-cell RNA-seq data with external single-cell RNA-seq datasets} UMI count matrices of single-cell RNA-seq data from Barbar et al. ${ }^{26}$ were downloaded from Synapse (syn21861229). Processed single-cell RNA-seq data objects corresponding to data from Wheeler et al. ${ }^{66}$ were requested from Zhaorong Li and Michael Wheeler. UMI count matrices of single-cell RNA-seq data from Hasel et al. ${ }^{52}$ were downloaded from GEO (GSE148611). Integration of NTC iAstrocytes from the CROP-seq experiment with the above previously published single-cell RNA-seq datasets was performed by first renormalizing each dataset with Seurat::SCTransform and re-running Seurat::RunPCA, then selecting features for integration using Seurat::SelectIntegrationFeatures (nfeatures = 3000) and Seurat::PrepSCTIntegration, and then performing data integration using reciprocal PCA with Seurat::FindIntegrationAnchors (normalization.method $=$ 'SCT', reduction $=$ 'rpca') and Seurat::IntegrateData (normalization.method $=$ 'SCT'), using NTC iAstrocytes as the reference dataset. The integrated PCA embeddings were then used for clustering (resolution $=0.4$ ) and UMAP. For integration with Barbar et $a ._{.}{ }^{26}$, the k.anchor parameter of Seurat::FindAnchors was set to 20; for Wheeler $e t$ $a l .{ }^{66}$, k.anchor was set to 50; for Hasel et al $^{52}$, k.anchor was set to 100 . Seurat::AddModuleScore was used to generate module expression scores for IRAS1 vs. IRAS2 markers in astrocytes from external single-cell datasets.

\section{Bulk RNA-seq of hiPSC-derived astrocytes generated in this study}

Alignment and quantification were performed using Salmon (version 1.4.082), with the -noLengthCorrection flag and an index generated from the human transcriptome (GRCh38, Gencode release 37). The $\mathrm{R}$ package Tximport (version 1.18.083) was used to obtain gene-level count estimates. Genes with zero counts across all samples were removed from the analysis. Differential gene expression analysis was performed with DESeq2 (version 1.30.1).

\section{Reanalysis of external bulk RNA-seq datasets}

For Guttenplan et al. ${ }^{9}$, we downloaded the table of DEGs induced by IL-1 $\alpha+\mathrm{TNF}+\mathrm{C} 1 \mathrm{q}$ in immunopanned astrocytes from wild-type mice provided in GSE143598. We used BioJupies ${ }^{93}$ to 
reanalyze bulk RNA-seq data and obtain differentially expressed genes from Perriot et al. ${ }^{94}$ (GSE120411; hiPSC-derived astrocytes treated with IL1 $\beta+T N F)$, Barbar et $_{\text {al. }}{ }^{26}$ (syn21861229; $\mathrm{CD} 49 \mathrm{f}+$ astrocytes sorted from cerebral organoids treated with vehicle control or IL$1 \alpha+\mathrm{TNF}+\mathrm{C} 1 \mathrm{q}$ ), and Anderson et al. ${ }^{45}$ (GSE76097; astrocyte-specific RNA from Stat3 cKO vs. wild-type mice subject to spinal cord injury). For Anderson et al., sample GSM1974209 was removed because it was an outlier in PCA visualization of the samples.

\section{Pathway enrichment analysis}

We used Enrichr ${ }^{95-97}$ to perform enrichment analysis of gene lists. For display of enrichment results against the TRRUST gene set library, terms corresponding to mouse gene sets were removed for genes lists derived from human astrocytes; for genes lists derived from mouse astrocytes, terms corresponding to human gene sets were removed.

\section{Flow cytometry}

Data from flow cytometry experiments were analyzed using FlowJo (version 10.7.1). Live cells were gated by plotting SSC-A vs. FSC-A and then single cells were gated by plotting FSC-H vs. FSC-A. For experiments involving CRISPRi knockdown, analysis was restricted to sgRNAtransduced cells (gating on the histogram of BFP fluorescence values). For antibody staining experiments where median fluorescence intensity (MFI) values were reported, the average MFI of unstained control samples were subtracted from the MFI of stained samples.

\section{Image segmentation and analysis}

We used CellProfiler (v3.15) ${ }^{98}$ to segment and quantify neuron viability, neuron GCaMP recordings, and iAstrocyte immunostaining images. For neuron viability, monochrome images of Hoechst and TO-PRO3 were segmented to nuclei and TO-PRO3+ objects, respectively; TOPRO3+ neurons (i.e. dead neurons) were determined by overlap of nuclei with TO-PRO3+ objects using the RelateObjects module in CellProfiler. For neuron GCaMP recordings, neuronal soma were segmented on the maximum intensity projection of all GCaMP images in the recording, nuclei were segmented on a single BFP image collected at the end of recording, and only nuclei that were contained within a GCaMP+ soma were retained using the RelatedObjects and FilterObjects modules in CellProfiler. The median GCaMP intensity per soma at each time point was then extracted for analysis. For each neuronal soma, $\Delta \mathrm{F}$ was calculated as $\mathrm{F}_{\mathrm{t}}-\mathrm{F}_{\mathrm{t}-1}$ and $F_{0}$ was calculated as the average of a 10-second rolling window around $F_{t}$.

For iAstrocyte immunostaining, the total image intensity of GFAP, S100 $\beta$, GLAST, and NFIA was calculated and divided by the total image intensity of Hoechst to normalize for cell number. For analysis of images from immunostaining of human neuropathological tissue, monochrome images of DAPI, VCAM1, C3, and GFAP staining were converted to probability maps of nuclei, VCAM1+ objects, C3+ objects, and GFAP+ objects respectively using ilastik ${ }^{99}$; annotations for representative nuclei, VCAM1+ objects, C3+ objects, and GFAP+ objects were inputted manually to train the classifier. The probability maps were then inputted into CellProfiler to segment nuclei, VCAM1+ objects, C3+ objects, and astrocytes (defined as nuclei-containing GFAP+ objects), and the VCAM1 or C3 staining status of astrocytes was determined by overlap of astrocytes with VCAM1+ or C3+ objects using the RelateObjects module in CellProfiler.

\section{Statistics and reproducibility}


Sample sizes were determined by referencing existing studies in the field. Major findings were validated using independent samples and orthogonal approaches. Numbers of replicates are listed in each figure. Randomization was not relevant to our study because no animals or human subjects were involved. 


\section{REFERENCES}

1. Sofroniew, M.V. \& Vinters, H.V. Astrocytes: biology and pathology. Acta Neuropathol 119, 7-35 (2010).

2. Escartin, C., et al. Reactive astrocyte nomenclature, definitions, and future directions. Nat Neurosci 24, 312-325 (2021).

3. Wang, Q., Tang, X.N. \& Yenari, M.A. The inflammatory response in stroke. $J$ Neuroimmunol 184, 53-68 (2007).

4. Hausmann, O.N. Post-traumatic inflammation following spinal cord injury. Spinal Cord 41, 369-378 (2003).

5. Ponath, G., Park, C. \& Pitt, D. The Role of Astrocytes in Multiple Sclerosis. Front Immunol 9, 217 (2018).

6. Heneka, M.T., et al. Neuroinflammation in Alzheimer's disease. Lancet Neurol 14, 388405 (2015).

7. Han, R.T., Kim, R.D., Molofsky, A.V. \& Liddelow, S.A. Astrocyte-immune cell interactions in physiology and pathology. Immunity 54, 211-224 (2021).

8. Liddelow, S.A., et al. Neurotoxic reactive astrocytes are induced by activated microglia. Nature 541, 481-487 (2017).

9. Guttenplan, K.A., et al. Knockout of reactive astrocyte activating factors slows disease progression in an ALS mouse model. Nat Commun 11, 3753 (2020).

10. Guttenplan, K.A., et al. Neurotoxic Reactive Astrocytes Drive Neuronal Death after Retinal Injury. Cell Rep 31, 107776 (2020).

11. Yun, S.P., et al. Block of A1 astrocyte conversion by microglia is neuroprotective in models of Parkinson's disease. Nat Med 24, 931-938 (2018).

12. Clarke, L.E., et al. Normal aging induces A1-like astrocyte reactivity. Proc Natl Acad Sci $U S$ A 115, E1896-E1905 (2018).

13. Herrmann, J.E., et al. STAT3 is a critical regulator of astrogliosis and scar formation after spinal cord injury. J Neurosci 28, 7231-7243 (2008).

14. Liu, Z., et al. Beneficial effects of gfap/vimentin reactive astrocytes for axonal remodeling and motor behavioral recovery in mice after stroke. Glia 62, 2022-2033 (2014).

15. Krencik, R. \& Zhang, S.C. Directed differentiation of functional astroglial subtypes from human pluripotent stem cells. Nat Protoc 6, 1710-1717 (2011). 
16. Zamanian, J.L., et al. Genomic analysis of reactive astrogliosis. J Neurosci 32, 63916410 (2012).

17. Kampmann, M. CRISPR-based functional genomics for neurological disease. Nat Rev Neurol 16, 465-480 (2020).

18. Tcw, J., et al. An Efficient Platform for Astrocyte Differentiation from Human Induced Pluripotent Stem Cells. Stem Cell Reports 9, 600-614 (2017).

19. Li, X., et al. Fast Generation of Functional Subtype Astrocytes from Human Pluripotent Stem Cells. Stem Cell Reports 11, 998-1008 (2018).

20. Abbott, N.J. Astrocyte-endothelial interactions and blood-brain barrier permeability. $J$ Anat 200, 629-638 (2002).

21. Allen, N.J. \& Eroglu, C. Cell Biology of Astrocyte-Synapse Interactions. Neuron 96, 697-708 (2017).

22. Verstraelen, P., et al. Pharmacological characterization of cultivated neuronal networks: relevance to synaptogenesis and synaptic connectivity. Cell Mol Neurobiol 34, 757-776 (2014).

23. Nakanishi, K., et al. Astrocytic contribution to functioning synapse formation estimated by spontaneous neuronal intracellular Ca2+ oscillations. Brain Res 659, 169-178 (1994).

24. Allen, N.J., et al. Astrocyte glypicans 4 and 6 promote formation of excitatory synapses via GluA1 AMPA receptors. Nature 486, 410-414 (2012).

25. Körber, N. \& Stein, V. In vivo imaging demonstrates dendritic spine stabilization by SynCAM 1. Sci Rep 6, 24241 (2016).

26. Barbar, L., et al. CD49f Is a Novel Marker of Functional and Reactive Human iPSCDerived Astrocytes. Neuron 107, 436-453.e412 (2020).

27. Williams, J.L., et al. Astrocyte-T cell crosstalk regulates region-specific neuroinflammation. Glia 68, 1361-1374 (2020).

28. Gimenez, M.A., Sim, J.E. \& Russell, J.H. TNFR1-dependent VCAM-1 expression by astrocytes exposes the CNS to destructive inflammation. J Neuroimmunol 151, 116-125 (2004).

29. Rosenman, S.J., Shrikant, P., Dubb, L., Benveniste, E.N. \& Ransohoff, R.M. Cytokineinduced expression of vascular cell adhesion molecule-1 (VCAM-1) by astrocytes and astrocytoma cell lines. J Immunol 154, 1888-1899 (1995).

30. Rubio, N., Sanz-Rodriguez, F. \& Arevalo, M.A. Up-regulation of the vascular cell adhesion molecule-1 (VCAM-1) induced by Theiler's murine encephalomyelitis virus infection of murine brain astrocytes. Cell Commun Adhes 17, 57-68 (2010). 
31. Kampmann, M. CRISPRi and CRISPRa Screens in Mammalian Cells for Precision Biology and Medicine. ACS Chem Biol 13, 406-416 (2018).

32. Castro, M.A., et al. Regulators of genetic risk of breast cancer identified by integrative network analysis. Nat Genet 48, 12-21 (2016).

33. Fletcher, M.N., et al. Master regulators of FGFR2 signalling and breast cancer risk. Nat Commun 4, 2464 (2013).

34. Campbell, T.M., et al. FGFR2 risk SNPs confer breast cancer risk by augmenting oestrogen responsiveness. Carcinogenesis 37, 741-750 (2016).

35. Krawczyk, M.C., et al. Human Astrocytes Exhibit Tumor Microenvironment-, Age-, and Sex-Related Transcriptomic Signatures. bioRxiv, 2021.2002.2025.432948 (2021).

36. Lambert, S.A., et al. The Human Transcription Factors. Cell 172, 650-665 (2018).

37. Manning, G., Whyte, D.B., Martinez, R., Hunter, T. \& Sudarsanam, S. The protein kinase complement of the human genome. Science 298, 1912-1934 (2002).

38. Liberti, S., et al. HuPho: the human phosphatase portal. FEBS J 280, 379-387 (2013).

39. Horlbeck, M.A., et al. Compact and highly active next-generation libraries for CRISPRmediated gene repression and activation. Elife 5 (2016).

40. Shih, V.F., Tsui, R., Caldwell, A. \& Hoffmann, A. A single NFкB system for both canonical and non-canonical signaling. Cell Res 21, 86-102 (2011).

41. Basak, S., et al. A fourth IkappaB protein within the NF-kappaB signaling module. Cell 128, 369-381 (2007).

42. Liu, T., Zhang, L., Joo, D. \& Sun, S.C. NF-кB signaling in inflammation. Signal Transduct Target Ther 2 (2017).

43. Cardinaux, J.R., Allaman, I. \& Magistretti, P.J. Pro-inflammatory cytokines induce the transcription factors C/EBPbeta and C/EBPdelta in astrocytes. Glia 29, 91-97 (2000).

44. Alonzi, T., et al. Essential role of STAT3 in the control of the acute-phase response as revealed by inducible gene inactivation [correction of activation] in the liver. Mol Cell Biol 21, 1621-1632 (2001).

45. Anderson, M.A., et al. Astrocyte scar formation aids central nervous system axon regeneration. Nature 532, 195-200 (2016).

46. Ben Haim, L., et al. The JAK/STAT3 pathway is a common inducer of astrocyte reactivity in Alzheimer's and Huntington's diseases. J Neurosci 35, 2817-2829 (2015).

47. Wang, Z.H., et al. C/EBP $\beta$ regulates delta-secretase expression and mediates pathogenesis in mouse models of Alzheimer's disease. Nat Commun 9, 1784 (2018). 
48. Strohmeyer, R., Shelton, J., Lougheed, C. \& Breitkopf, T. CCAAT-enhancer binding protein- $\beta$ expression and elevation in Alzheimer's disease and microglial cell cultures. PLoS One 9, e86617 (2014).

49. Feng, H., Zhang, Y.B., Gui, J.F., Lemon, S.M. \& Yamane, D. Interferon regulatory factor 1 (IRF1) and anti-pathogen innate immune responses. PLoS Pathog 17, e1009220 (2021).

50. Lehtonen, A., Matikainen, S. \& Julkunen, I. Interferons up-regulate STAT1, STAT2, and IRF family transcription factor gene expression in human peripheral blood mononuclear cells and macrophages. J Immunol 159, 794-803 (1997).

51. $\mathrm{Ng}$, S.L., et al. IкB kinase epsilon (IKK(epsilon)) regulates the balance between type I and type II interferon responses. Proc Natl Acad Sci U S A 108, 21170-21175 (2011).

52. Hasel, P., Rose, I.V.L., Sadick, J.S., Kim, R.D. \& Liddelow, S.A. Neuroinflammatory astrocyte subtypes in the mouse brain. Nat Neurosci (2021).

53. Gan, W., et al. LATS suppresses mTORC1 activity to directly coordinate Hippo and mTORC1 pathways in growth control. Nat Cell Biol 22, 246-256 (2020).

54. He, L., et al. mTORC1 Promotes Metabolic Reprogramming by the Suppression of GSK3-Dependent Foxk1 Phosphorylation. Mol Cell 70, 949-960.e944 (2018).

55. Datlinger, P., et al. Pooled CRISPR screening with single-cell transcriptome readout. Nat Methods 14, 297-301 (2017).

56. Banks, T.A., et al. A lymphotoxin-IFN-beta axis essential for lymphocyte survival revealed during cytomegalovirus infection. J Immunol 174, 7217-7225 (2005).

57. Weichhart, T., Hengstschläger, M. \& Linke, M. Regulation of innate immune cell function by mTOR. Nat Rev Immunol 15, 599-614 (2015).

58. Balamurugan, K., et al. The tumour suppressor C/EBP $\delta$ inhibits FBXW7 expression and promotes mammary tumour metastasis. EMBO J 29, 4106-4117 (2010).

59. Heinrich, P.C., Castell, J.V. \& Andus, T. Interleukin-6 and the acute phase response. Biochem J 265, 621-636 (1990).

60. Sonderegger, I., et al. GM-CSF mediates autoimmunity by enhancing IL-6-dependent Th17 cell development and survival. J Exp Med 205, 2281-2294 (2008).

61. Metzemaekers, M., Vanheule, V., Janssens, R., Struyf, S. \& Proost, P. Overview of the Mechanisms that May Contribute to the Non-Redundant Activities of Interferon-Inducible CXC Chemokine Receptor 3 Ligands. Front Immunol 8, 1970 (2017).

62. Wang, Y., van Boxel-Dezaire, A.H., Cheon, H., Yang, J. \& Stark, G.R. STAT3 activation in response to IL-6 is prolonged by the binding of IL-6 receptor to EGF receptor. Proc Natl Acad Sci U S A 110, 16975-16980 (2013). 
63. Au-Yeung, N., Mandhana, R. \& Horvath, C.M. Transcriptional regulation by STAT1 and STAT2 in the interferon JAK-STAT pathway. JAKSTAT 2, e23931 (2013).

64. Hungness, E.S., et al. Transcription factors C/EBP-beta and -delta regulate IL-6 production in IL-1beta-stimulated human enterocytes. J Cell Physiol 192, 64-70 (2002).

65. Stuart, T., et al. Comprehensive Integration of Single-Cell Data. Cell 177, 18881902.e1821 (2019).

66. Wheeler, M.A., et al. MAFG-driven astrocytes promote CNS inflammation. Nature 578, 593-599 (2020).

67. Heppner, F.L., Ransohoff, R.M. \& Becher, B. Immune attack: the role of inflammation in Alzheimer disease. Nat Rev Neurosci 16, 358-372 (2015).

68. Eikelenboom, P., Hack, C.E., Rozemuller, J.M. \& Stam, F.C. Complement activation in amyloid plaques in Alzheimer's dementia. Virchows Arch B Cell Pathol Incl Mol Pathol 56, 259262 (1989).

69. Abraham, C.R., Selkoe, D.J. \& Potter, H. Immunochemical identification of the serine protease inhibitor alpha 1-antichymotrypsin in the brain amyloid deposits of Alzheimer's disease. Cell 52, 487-501 (1988).

70. Habib, N., et al. Disease-associated astrocytes in Alzheimer's disease and aging. Nat Neurosci 23, 701-706 (2020).

71. Liu, F. \& McCullough, L.D. Inflammatory responses in hypoxic ischemic encephalopathy. Acta Pharmacol Sin 34, 1121-1130 (2013).

72. Reichenbach, N., et al. Inhibition of Stat3-mediated astrogliosis ameliorates pathology in an Alzheimer's disease model. EMBO Mol Med 11 (2019).

73. Clarke, P., Zhuang, Y., Berens, H.M., Leser, J.S. \& Tyler, K.L. Interferon Beta Contributes to Astrocyte Activation in the Brain following Reovirus Infection. J Virol 93 (2019).

74. Sanmarco, L.M., et al. Gut-licensed IFN $\gamma$. Nature 590, 473-479 (2021).

75. Rothhammer, V., et al. Type I interferons and microbial metabolites of tryptophan modulate astrocyte activity and central nervous system inflammation via the aryl hydrocarbon receptor. Nat Med 22, 586-597 (2016).

76. Hindinger, C., et al. IFN- $\gamma$ signaling to astrocytes protects from autoimmune mediated neurological disability. PLoS One 7, e42088 (2012).

77. Miyaoka, Y., et al. Isolation of single-base genome-edited human iPS cells without antibiotic selection. Nat Methods 11, 291-293 (2014). 
78. Tian, R., et al. CRISPR Interference-Based Platform for Multimodal Genetic Screens in Human iPSC-Derived Neurons. Neuron 104, 239-255 e212 (2019).

79. Li, X.L., et al. Highly efficient genome editing via CRISPR-Cas9 in human pluripotent stem cells is achieved by transient BCL-XL overexpression. Nucleic Acids Res 46, 10195-10215 (2018).

80. Cheng, C., Fass, D.M., Folz-Donahue, K., MacDonald, M.E. \& Haggarty, S.J. Highly Expandable Human iPS Cell-Derived Neural Progenitor Cells (NPC) and Neurons for Central Nervous System Disease Modeling and High-Throughput Screening. Curr Protoc Hum Genet 92, 21.28.21-21.28.21 (2017).

81. Gilbert, L.A., et al. Genome-Scale CRISPR-Mediated Control of Gene Repression and Activation. Cell 159, 647-661 (2014).

82. Neal, E.H., et al. A Simplified, Fully Defined Differentiation Scheme for Producing Blood-Brain Barrier Endothelial Cells from Human iPSCs. Stem Cell Reports 12, 1380-1388 (2019).

83. Fernandopulle, M.S., et al. Transcription Factor-Mediated Differentiation of Human iPSCs into Neurons. Curr Protoc Cell Biol 79, e51 (2018).

84. Niu, J., et al. Oligodendroglial ring finger protein Rnf43 is an essential injury-specific regulator of oligodendrocyte maturation. Neuron (2021).

85. Lachmann, A., et al. Massive mining of publicly available RNA-seq data from human and mouse. Nat Commun 9, 1366 (2018).

86. Lachmann, A., Xie, Z. \& Ma'ayan, A. Elysium: RNA-seq Alignment in the Cloud. bioRxiv, 382937 (2018).

87. Zhang, Y., Parmigiani, G. \& Johnson, W.E.: batch effect adjustment for RNA-seq count data. NAR Genom Bioinform 2, lqaa078 (2020).

88. Hill, A.J., et al. On the design of CRISPR-based single-cell molecular screens. Nat Methods 15, 271-274 (2018).

89. Gaublomme, J.T., et al. Nuclei multiplexing with barcoded antibodies for single-nucleus genomics. Nat Commun 10, 2907 (2019).

90. Hafemeister, C. \& Satija, R. Normalization and variance stabilization of single-cell RNAseq data using regularized negative binomial regression. Genome Biol 20, 296 (2019).

91. McInnes, L., Healy, J. \& Melville, J. UMAP: Uniform Manifold Approximation and Projection for Dimension Reduction. arXiv, 1802.03426 (2020).

92. Gu, Z., Eils, R. \& Schlesner, M. Complex heatmaps reveal patterns and correlations in multidimensional genomic data. Bioinformatics 32, 2847-2849 (2016). 
93. Torre, D., Lachmann, A. \& Ma'ayan, A. BioJupies: Automated Generation of Interactive Notebooks for RNA-Seq Data Analysis in the Cloud. Cell Syst 7, 556-561.e553 (2018).

94. Perriot, S., et al. Human Induced Pluripotent Stem Cell-Derived Astrocytes Are Differentially Activated by Multiple Sclerosis-Associated Cytokines. Stem Cell Reports 11, 1199-1210 (2018).

95. Chen, E.Y., et al. Enrichr: interactive and collaborative HTML5 gene list enrichment analysis tool. BMC Bioinformatics 14, 128 (2013).

96. Kuleshov, M.V., et al. Enrichr: a comprehensive gene set enrichment analysis web server 2016 update. Nucleic Acids Res 44, W90-97 (2016).

97. Xie, Z., et al. Gene Set Knowledge Discovery with Enrichr. Curr Protoc 1, e90 (2021).

98. McQuin, C., et al. CellProfiler 3.0: Next-generation image processing for biology. PLoS Biol 16, e2005970 (2018).

99. Berg, S., et al. ilastik: interactive machine learning for (bio)image analysis. Nat Methods 16, 1226-1232 (2019).

100. Liberzon, A., et al. The Molecular Signatures Database (MSigDB) hallmark gene set collection. Cell Syst 1, 417-425 (2015).

101. Han, H., et al. TRRUST v2: an expanded reference database of human and mouse transcriptional regulatory interactions. Nucleic Acids Res 46, D380-D386 (2018).

102. Kelley, K.W., Nakao-Inoue, H., Molofsky, A.V. \& Oldham, M.C. Variation among intact tissue samples reveals the core transcriptional features of human CNS cell classes. Nat Neurosci 21, 1171-1184 (2018).

103. Huang, R., et al. The NCATS BioPlanet - An Integrated Platform for Exploring the Universe of Cellular Signaling Pathways for Toxicology, Systems Biology, and Chemical Genomics. Front Pharmacol 10, 445 (2019).

104. Cao, J., et al. A human cell atlas of fetal gene expression. Science 370 (2020). 


\section{FIGURES}

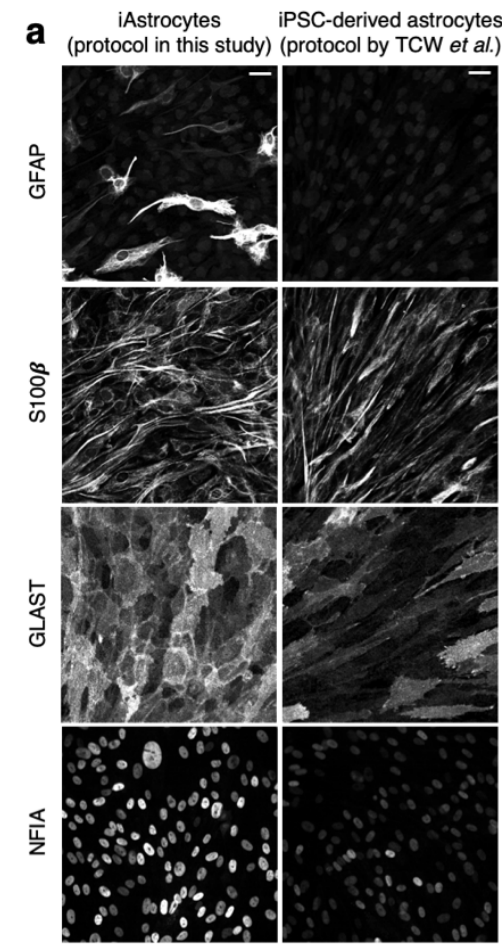

\section{b}
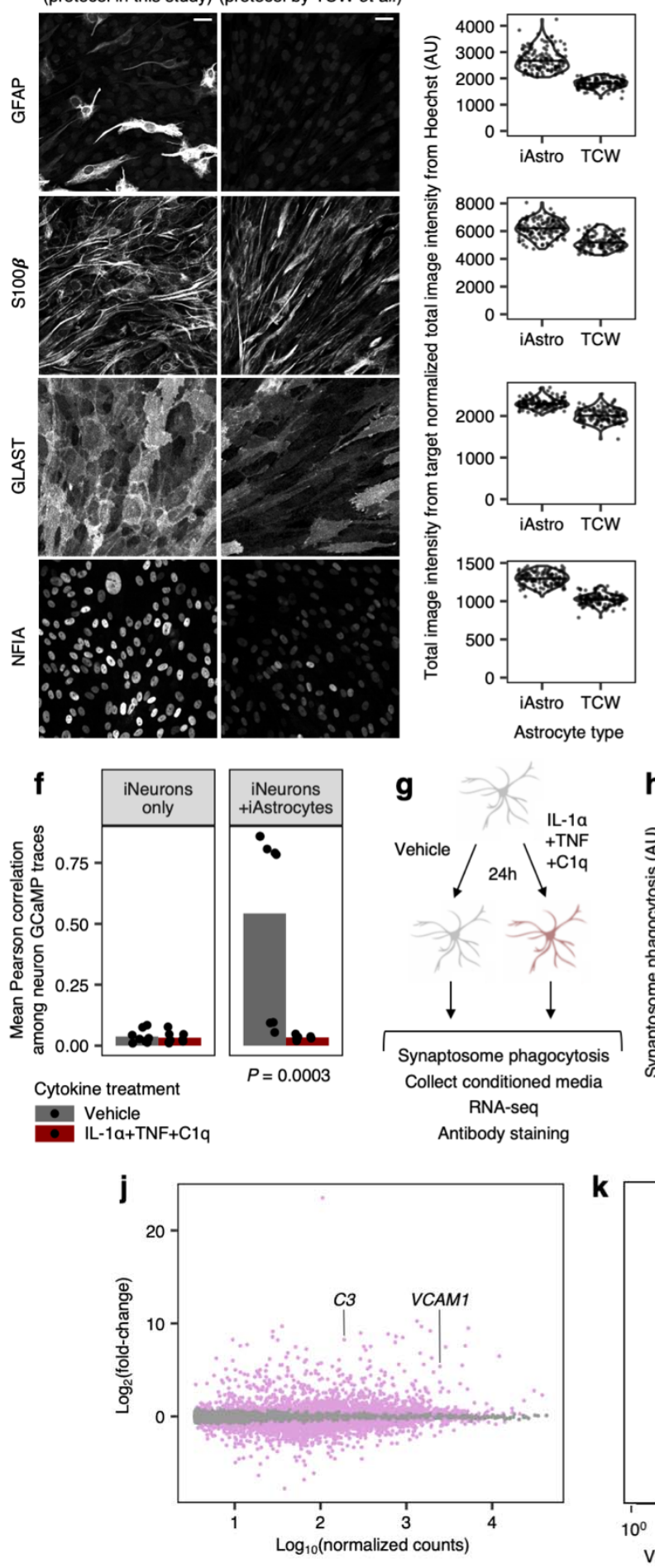

Differential expression $\left(P_{\text {adjusted }}<0.05\right)$

- Non-significant Significant
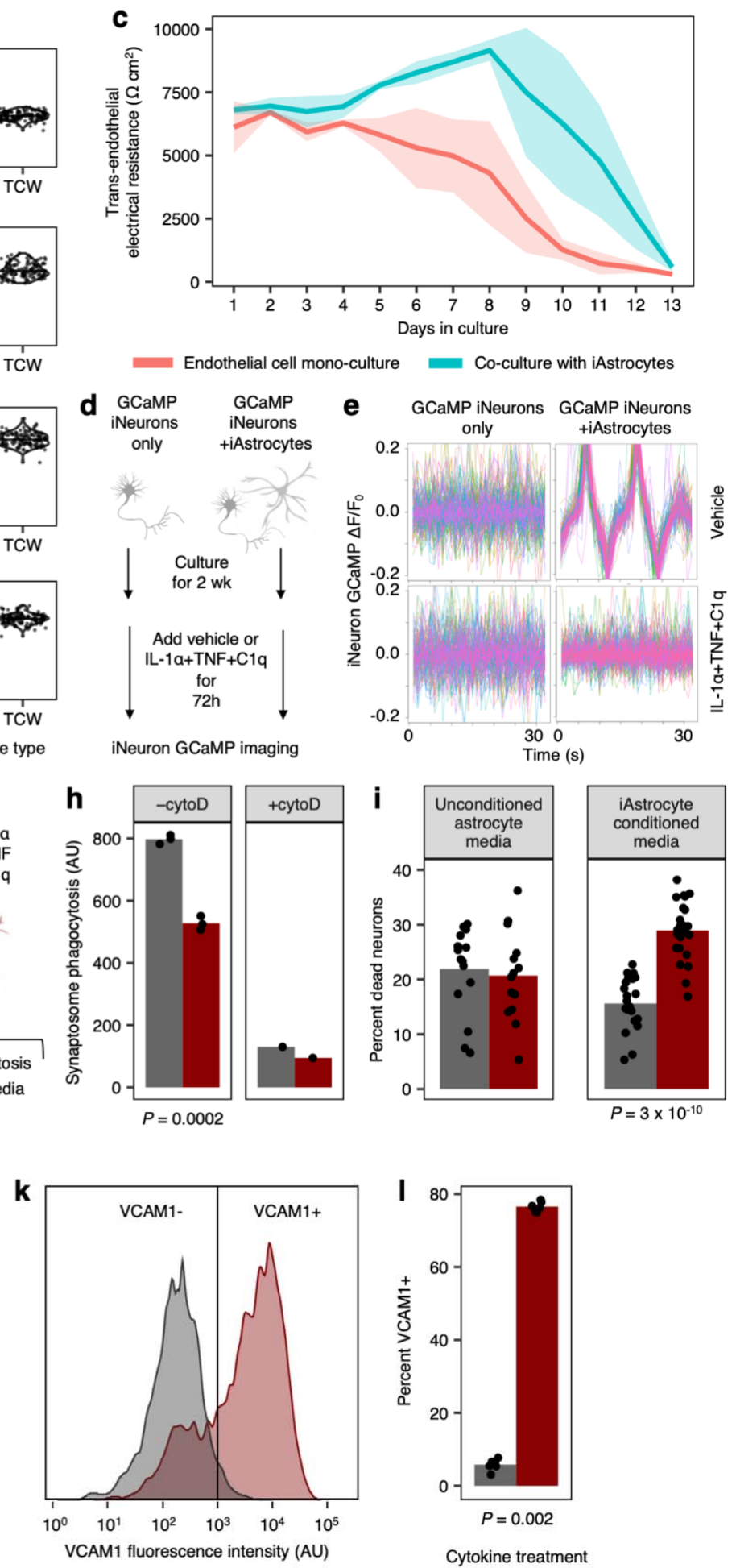

Cytokine treatment - Vehicle

$\mathrm{IL}-1 \mathrm{a}+\mathrm{TNF}+\mathrm{C} 1 \mathrm{q}$

Fig. 1 (Legend overleaf) 
Fig. 1 | iPSC-derived astrocytes (iAstrocytes) perform canonical astrocyte functions and recapitulate key aspects of inflammatory reactivity upon treatment with IL-1 $\alpha+\mathbf{T N F}+\mathrm{C1q}$. a, Representative immunofluorescence micrographs of astrocyte marker expression in iAstrocytes vs. iPSC-derived astrocytes generated using the protocol from $T C W$ et al. ${ }^{18}$ Scale bar: $60 \mu \mathrm{m}$. b, Quantification of astrocyte marker expression measured by immunofluorescence (see Methods) in iAstrocytes (iAstro) vs. astrocytes generated using the $T C W$ et al. protocol (TCW); data points represent fields of view collected over two experimental replicates. c, Barrier integrity of brain endothelial-like cells cultured alone or with iAstrocytes over time; lines represent group means ( $n=5-6$ per condition), and shaded bands around lines represent $95 \%$ confidence intervals. d, Workflow schematic of calcium imaging experiments using GCaMPexpressing iPSC-derived neurons (iNeurons) cultured alone or with iAstrocytes. e, Neuronal calcium activity traces of GCaMP iNeurons co-cultured with iAstrocytes treated with vehicle control or IL-1 $\alpha+\mathrm{TNF}+\mathrm{C} 1 \mathrm{q}$; traces from individual neurons are colored differently and overlaid. f, Quantification of synchrony between neuronal calcium activity traces in iNeuron monocultures or iNeuron + iAstrocyte co-cultures treated with vehicle control or IL- $1 \alpha+\mathrm{TNF}+\mathrm{C} 1 \mathrm{q}(n$ $=8$ experimental replicates for each condition). $\mathbf{g}$, Workflow schematic of experiments assessing inflammatory reactivity induced by IL-1 $\alpha+\mathrm{TNF}+\mathrm{C} 1 \mathrm{q}$ in iAstrocytes. $\mathbf{h}$, Phagocytosis of pHrodolabeled rat synaptosomes (median pHrodo fluorescence intensity measured by flow cytometry) by iAstrocytes treated with vehicle control or IL- $1 \alpha+\mathrm{TNF}+\mathrm{C} 1 \mathrm{q}$ in the absence $(n=3)$ or presence $(n=1)$ of cytochalasin $\mathrm{D}$ (cytoD); $n$ is number of experimental replicates. $\mathbf{i}$, Percentage of dead cells (measured by TO-PRO-3 permeability) for iNeurons incubated with unconditioned astrocyte media $+/-$ IL- $1 \alpha+\mathrm{TNF}+\mathrm{C} 1 \mathrm{q}$ or astrocyte media conditioned by iAstrocytes treated with vehicle control or IL-1 $\alpha+\mathrm{TNF}+\mathrm{C} 1 \mathrm{q}$ ( $n=23-24$ experimental replicates for each condition). $\mathbf{j}$, Log-scaled fold change vs. average expression of differentially expressed genes (measured by RNA-seq) induced by IL- $1 \alpha+\mathrm{TNF}+\mathrm{C} 1 \mathrm{q}$ in iAstrocytes $(n=3$ experimental replicates for each condition). k, Representative histogram of cell-surface VCAM1 levels (measured by flow cytometry) in iAstrocytes treated with vehicle control or IL-1 $\alpha+\mathrm{TNF}+\mathrm{C} 1 \mathrm{q}$. l, Percent of VCAM1+ iAstrocytes (measured by flow cytometry) after treatment with vehicle control or IL$1 \alpha+\mathrm{TNF}+\mathrm{C} 1 \mathrm{q}$ ( $n=3$ experimental replicates for each condition). The two-sided Mann-Whitney U-test was used in panels $\mathbf{f}, \mathbf{i}$, and $\mathbf{l}$. The two-sided Student's t-test was used in panel h. 
a
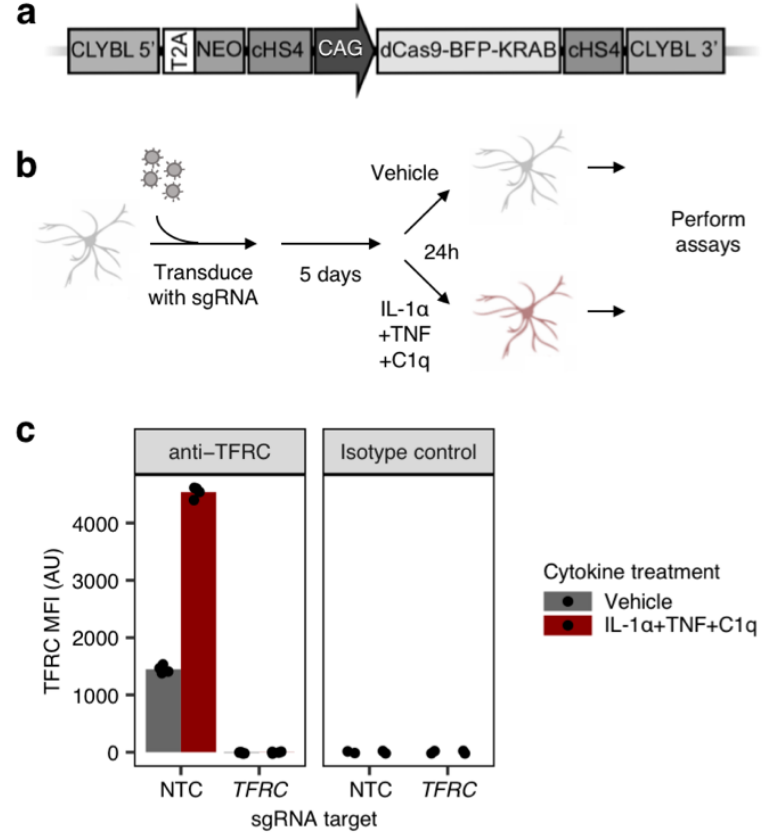

Fig. 2 | CRISPR interference (CRISPRi) platform in iAstrocytes. a, Schematic of CRISPRi machinery cassette. b, Workflow schematic of experiments involving lentiviral sgRNA transduction for CRISPRi knockdown. c, Cell-surface TFRC levels (measured by flow cytometry using an anti-TFRC antibody or isotype control; MFI: median fluorescence intensity) in iAstrocytes transduced with a non-targeting control (NTC) sgRNA or a sgRNA targeting TFRC, treated with vehicle control or IL-1 $\alpha+\mathrm{TNF}+\mathrm{C} 1 \mathrm{q}(n=4$ for anti-TFRC, $n=2$ for isotype control; $n$ is number of experimental replicates) . 
bioRxiv preprint doi: https://doi.org/10.1101/2021.08.23.457400; The copyright holder for this preprint (which was not certified by peer review)

is the author/funder, who has granted bioRxiv a license to display the preprint in perpetuity. It is made available under a CC-BY 4.0 International license .
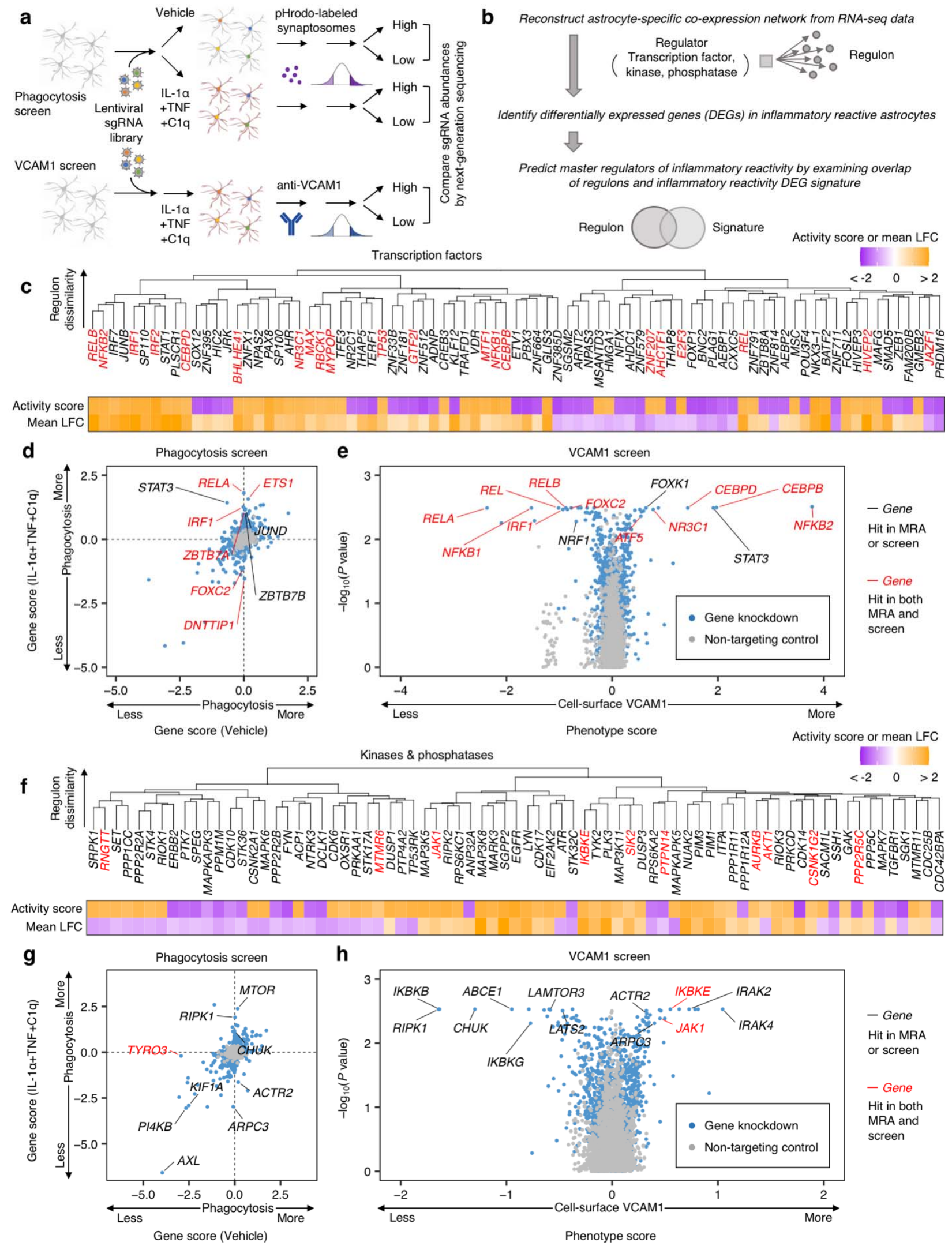

- Gene

Hit in MRA

or screen

- Gene

Hit in both

MRA and

screen

Fig. 3 (Legend overleaf) 
Fig. 3 | CRISPRi screening and master regulator analysis uncover regulators of

inflammatory reactivity. a, Workflow schematic of synaptosome phagocytosis and cell-surface VCAM1 CRISPRi screens ( $n=2$ experimental replicates for each condition). b, Workflow schematic of bioinformatic master regulator analysis (MRA). c, f, Clustering of transcription factors (c) or kinases and phosphatases (f) predicted to regulate inflammatory reactivity based on regulon overlap (see Methods); the activity score and regulon mean log-fold-change (LFC) associated with each predicted regulator (see Methods) are shown below the dendrogram. d, g, Scatterplot of gene scores (see Methods) of transcription factors (d) or the druggable genome (g) from synaptosome phagocytosis screens on iAstrocytes treated with vehicle control vs. IL$1 \alpha+\mathrm{TNF}+\mathrm{C} 1 \mathrm{q} . \mathbf{e}, \mathbf{h}$, Volcano plot of phenotype scores and associated log-scaled $P$ values (see Methods) of transcription factors (e) or the druggable genome (h) from cell-surface VCAM1 screens on iAstrocytes treated with IL-1 $\alpha+\mathrm{TNF}+\mathrm{C} 1 \mathrm{q}$. 


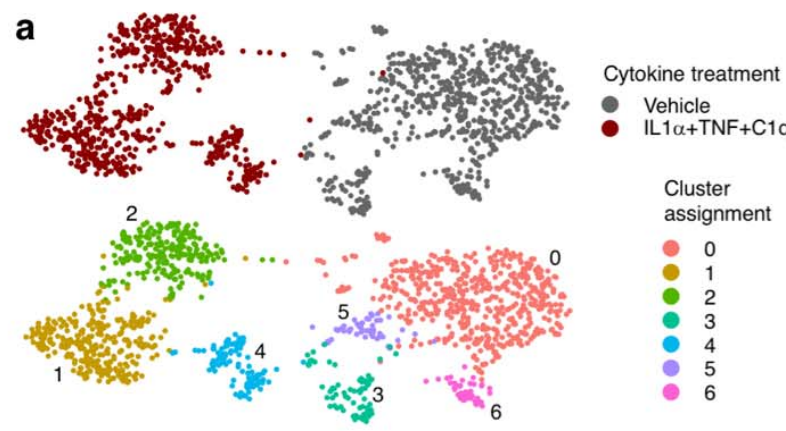

c
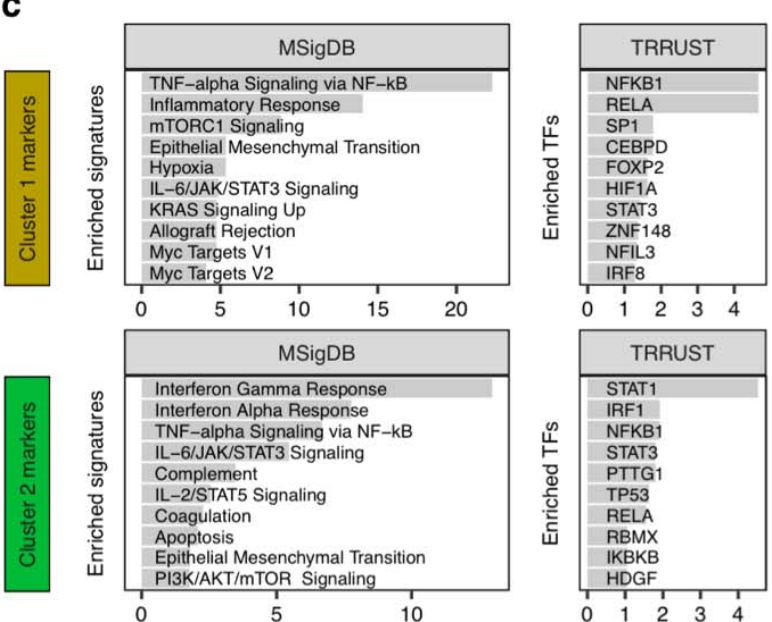

f
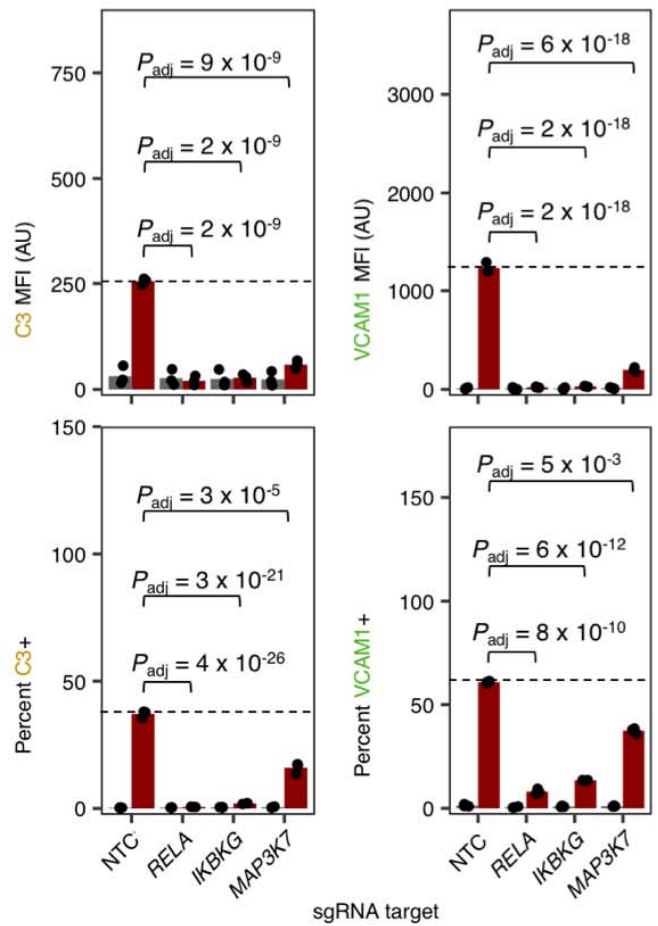

b

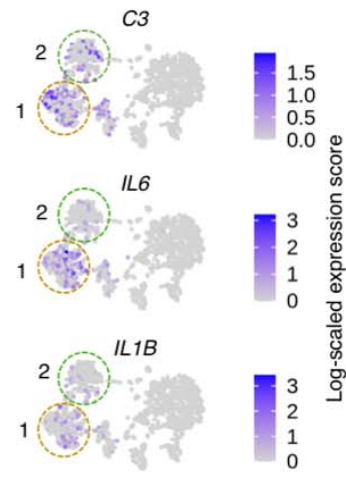

d
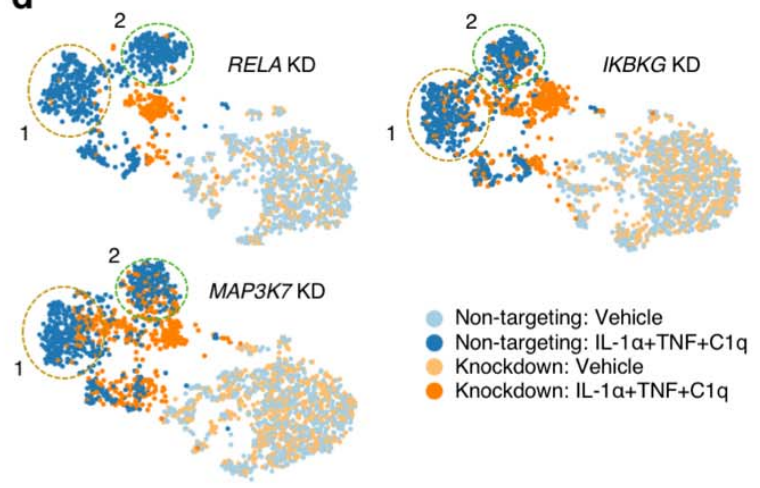

Non-targeting: Vehicle

Non-targeting: IL-1a+TNF+C1q

Knockdown: Vehicle

Knockdown: IL-1a+TNF+C1q
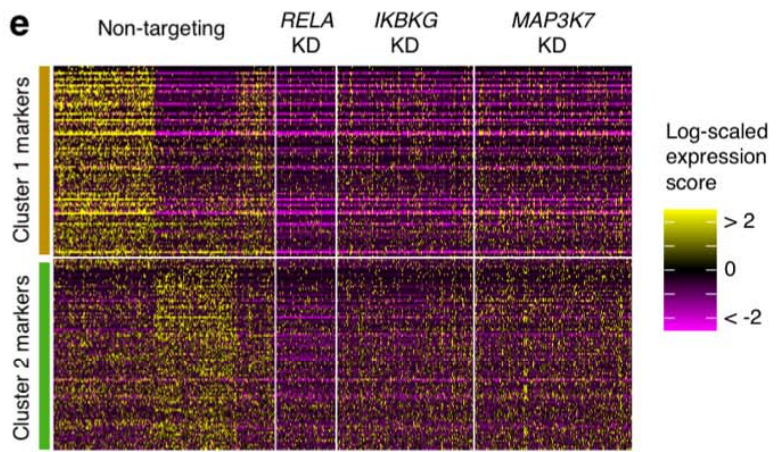

- Non-targeting:

$\mathrm{IL}-1 \mathrm{a}+\mathrm{TNF}+\mathrm{C} 1 \mathrm{q}$
Knockdown: IL-1a+TNF+C1q

IL-1a
+ TNF

$+\mathrm{C} 1 \mathrm{q}$

Fig. 4 (legend overleaf) 
Fig. 4 | CROP-seq of iAstrocytes reveals two distinct inflammatory reactive states dependent on the canonical NF-kB pathway. a, Uniform manifold approximation projection (UMAP) of iAstrocytes transduced with a non-targeting control (NTC) sgRNA treated with vehicle control or IL-1 $\alpha+\mathrm{TNF}+\mathrm{C} 1 \mathrm{q}$, colored by cytokine treatment or cluster assignment. b, Visualization of transcript levels of selected Cluster 1 and Cluster 2 markers in the iAstrocytes shown in panel a, overlaid onto the same UMAP embedding. c, Cellular pathway (MSigDB ${ }^{100}$ ) and upstream transcription factor (TRRUST ${ }^{101}$ ) enrichment analysis of Cluster 1 and Cluster 2 markers; TF - transcription factor. d, Aligned UMAP embedding (see Methods) of NTC sgRNA transduced iAstrocytes with iAstrocytes transduced with sgRNAs knocking down RELA, $I K B K G$, or MAP3K7. e, Heatmap of Cluster 1 and Cluster 2 marker transcript levels in IL$1 \alpha+\mathrm{TNF}+\mathrm{C} 1 \mathrm{q}$-treated NTC sgRNA iAstrocytes compared to IL-1 $\alpha+\mathrm{TNF}+\mathrm{C} 1 \mathrm{q}$-treated $R E L A$, $I K B K G$, and $M A P 3 K 7$ sgRNA iAstrocytes. f, VCAM1/C3 levels (MFI: median fluorescence intensity) or percent positive cells measured by flow cytometry in NTC, RELA, IKBKG, or $M A P 3 K 7$ sgRNA iAstrocytes treated with vehicle control or IL- $1 \alpha+\mathrm{TNF}+\mathrm{C} 1 \mathrm{q}(n=3$ experimental replicates for each condition). Statistical significance of differences between IL$1 \alpha+\mathrm{TNF}+\mathrm{C} 1 \mathrm{q}$-treated NTC iAstrocytes (marked by dotted line) and knockdown iAstrocytes was determined by linear regression for MFI values or beta regression for percentages (see Methods); comparisons with statistically significant differences are marked; adjustment of $P$ values for multiple testing ( $P_{\text {adj }}$; Holm's method) is performed per family of tests, i.e. all comparisons made within a plot. In panels $\mathbf{b}$ and $\mathbf{d}$, NTC sgRNA astrocytes in Cluster 1 or Cluster 2 are circled by colored dotted lines. 

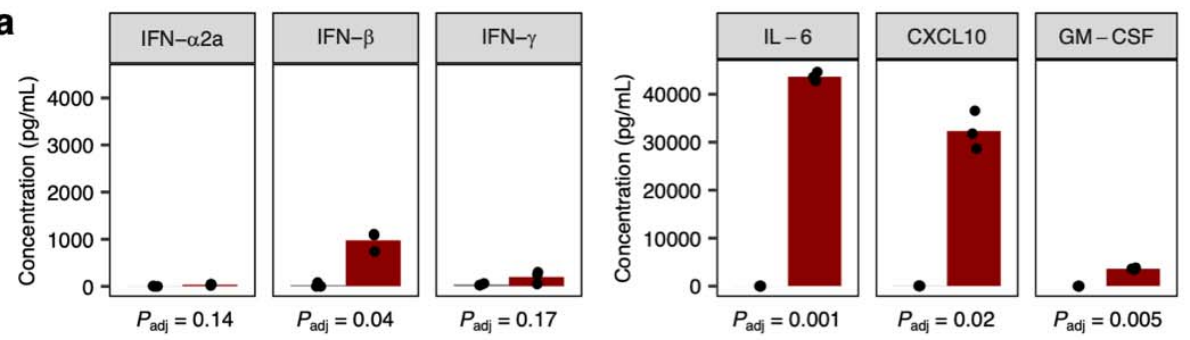

Cytokine treatment

- Vehicle

IL-1a+TNF+C1q

b
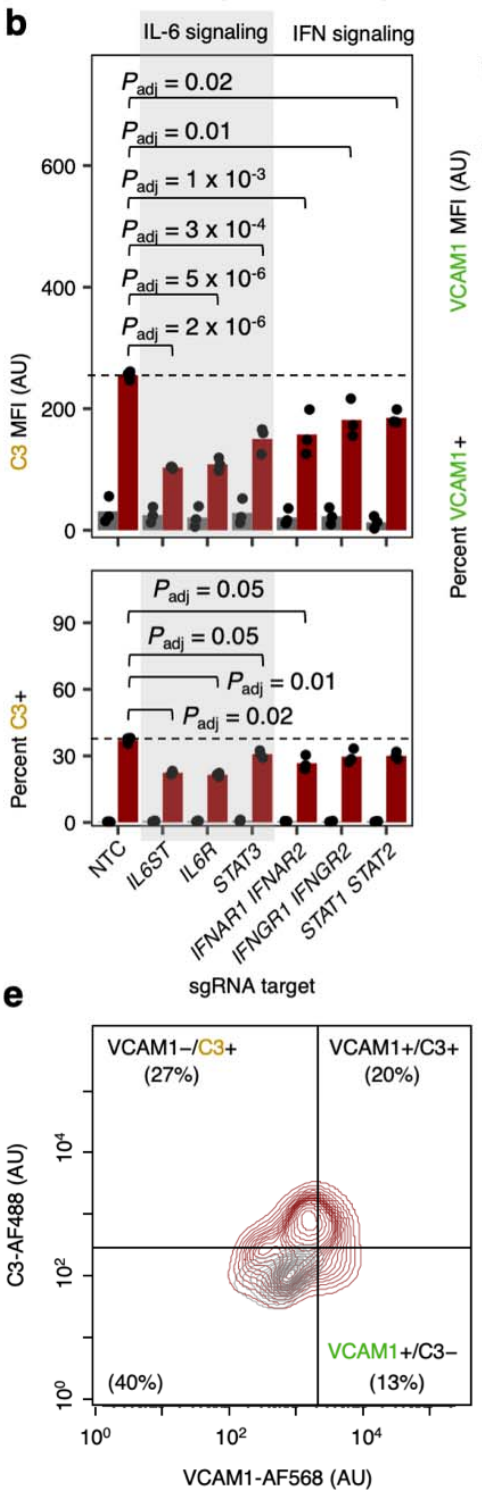

Cytokine treatment - Vehicle IL-1a+TNF+C1q
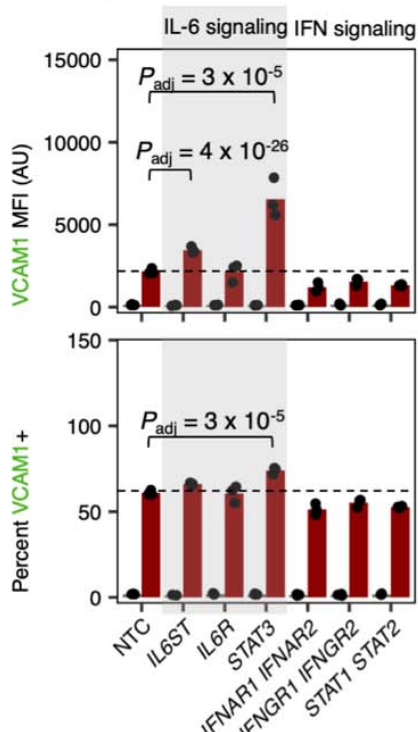

sgRNA target

\section{f}
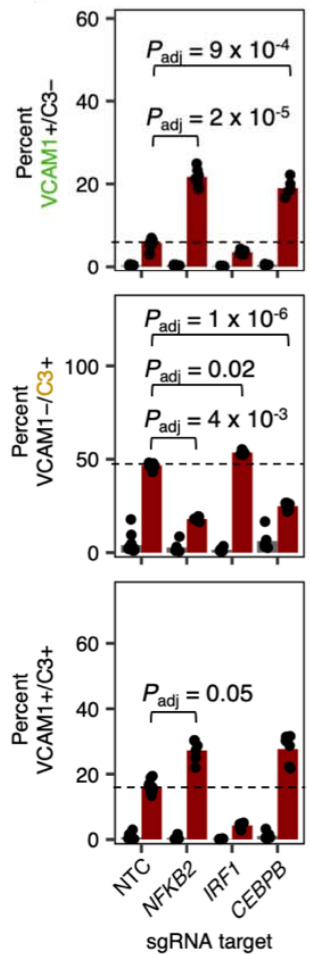

c IRAS2

CSF2 (GM-CSF)

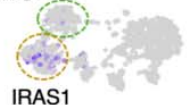

IRAS1

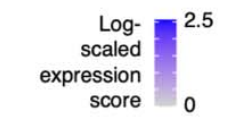

IRAS2

CXCL10

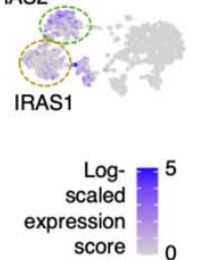

Cytokine treatment

- Vehicle

IL-1a+TNF+C1q

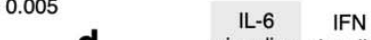
signaling signaling
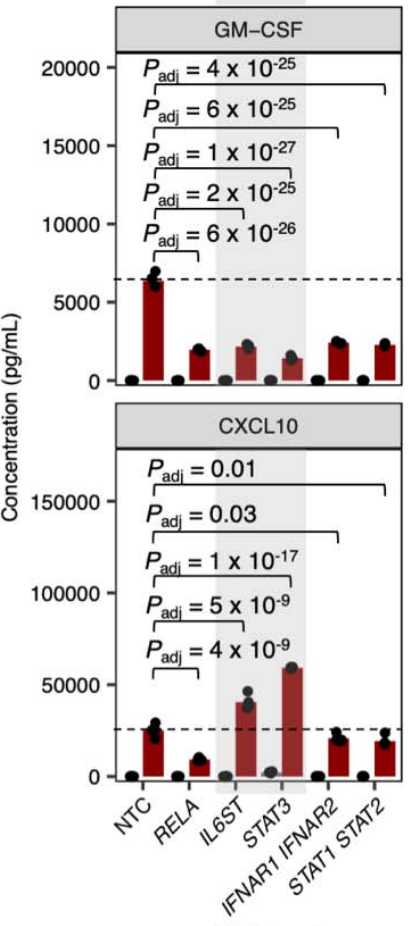

sgRNA target g

Primary

transcriptional

response

Autocrineparacrine

signaling and secondary transcriptional response

Inflammatory reactive astrocyte states (IRAS)

AS)
and literature
IL-1a+TNF+C1q

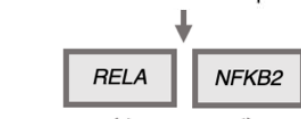

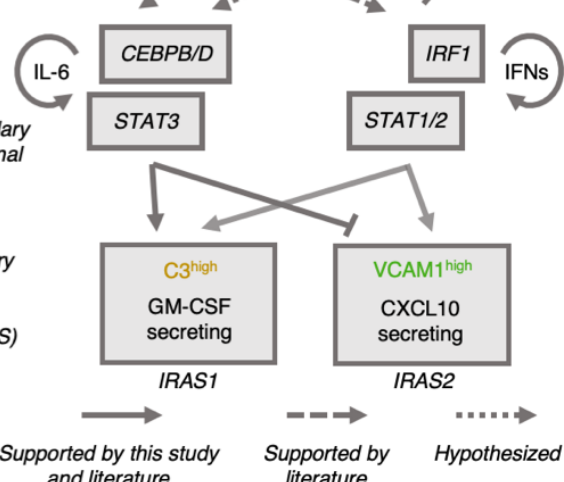
literature

Fig. 5 (legend overleaf) 
Fig. 5 | IL-6 and interferons act in an autocrine-paracrine manner to drive distinct inflammatory reactive states. a, Cytokine concentrations in conditioned media (measured by multi-spot electrochemiluminescence) from iAstrocytes treated with vehicle control or IL$1 \alpha+\mathrm{TNF}+\mathrm{C} 1 \mathrm{q}$ ( $n=3$ experimental replicates for each condition). b, VCAM1/C3 levels (MFI: median fluorescence intensity) or percent positive cells measured by flow cytometry from NTC sgRNA iAstrocytes compared to iAstrocytes transduced with sgRNAs knocking down genes involved in IL-6 or interferon (IFN) signaling; $n=3$ experimental replicates per condition. c, Transcript levels of CSF2 and GM-CSF overlaid onto the UMAP embedding from Fig. 3a. d, Concentration of GM-CSF or CXCL10 in conditioned media from iAstrocytes transduced with sgRNAs knocking down genes involved in IL-6 or IFN signaling; $n=4$ experimental replicates per condition. e, Representative scatterplot of VCAM1 and C3 levels (measured by flow cytometry) in iAstrocytes treated with vehicle control or IL-1 $\alpha+\mathrm{TNF}+\mathrm{C} 1 \mathrm{q}$. f, Percent VCAM1+/C3-, VCAM1-/C3+, or VCAM1+/C3+ cells (measured by flow cytometry) in NTC, $N F K B 2, I R F 1$, or $C E B P B \operatorname{sgRNA}$ iAstrocytes treated with vehicle control or IL-1 $\alpha+\mathrm{TNF}+\mathrm{C} 1 \mathrm{q}$ ( $n=6$ experimental replicates for each condition). $\mathrm{g}$, Model schematic of transcription factors and signaling pathways controlling inflammatory reactivity. Statistical significance of differences between IL-1 $\alpha+\mathrm{TNF}+\mathrm{C} 1 \mathrm{q}$-treated NTC iAstrocytes (marked by dotted line) and knockdown iAstrocytes was determined by linear regression for MFI values or beta regression for percentages (see Methods); comparisons with statistically significant differences are marked; adjustment of $P$ values for multiple testing ( $P_{\text {adj }}$; Holm's method) is performed per family of tests, i.e. all comparisons made within a plot. 
a
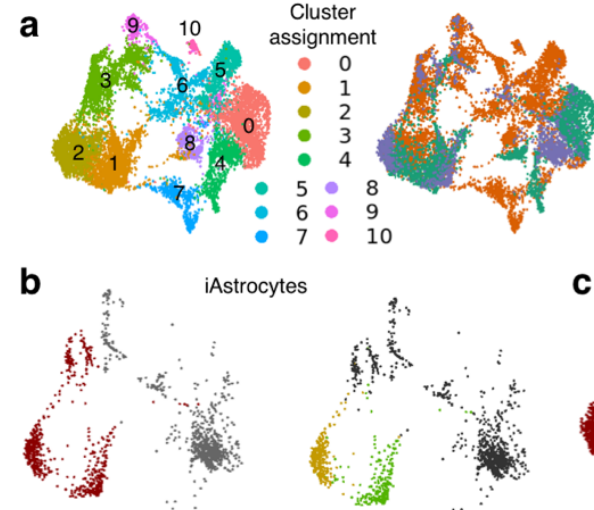

Cytokine treatment - Vehicle

IL-1a+TNF+C1q

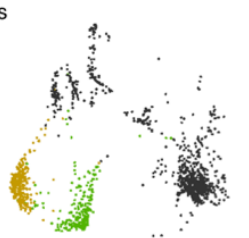

Fig. 4a cluster assignment - Fig. 4a Cluster 1 (IRAS1)

- Fig. 4a Cluster 2 (IRAS2)

C

Barbar et al. astrocytes

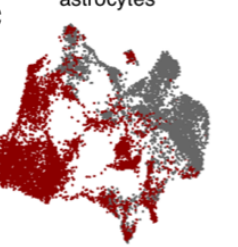

Cytokine treatment - Vehicle

- IL-1a+TNF+C1q

e

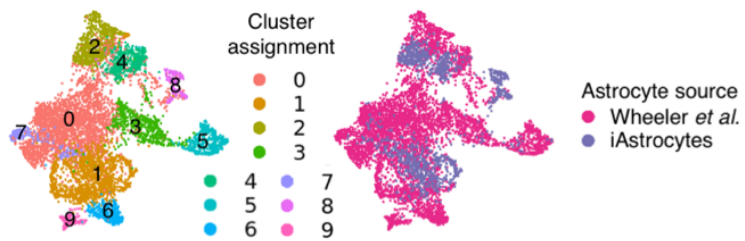

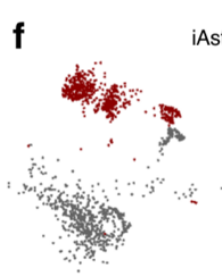

Cytokine treatment - Vehicle

- IL-1a+TNF+C1q
Astrocytes

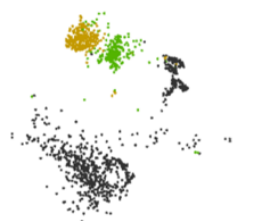

Fig. 3a cluster assignment

- Fig. 4a Cluster 1 (IRAS1)

- Fig. 4a Cluster 2 (IRAS2) g

Wheeler et al.

astrocytes

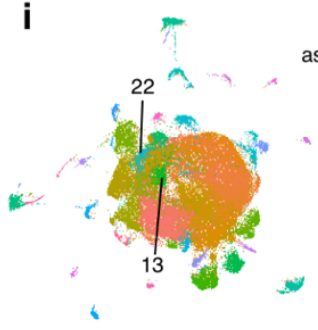

j

iAstrocytes

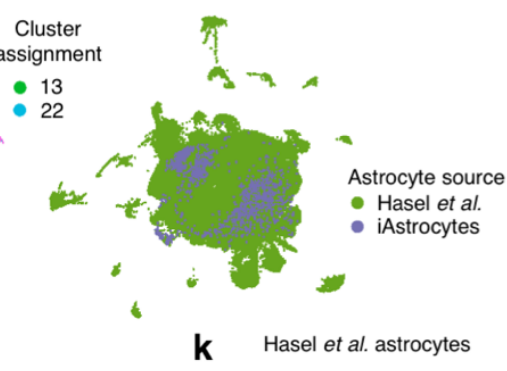

k Hasel et al. astrocytes

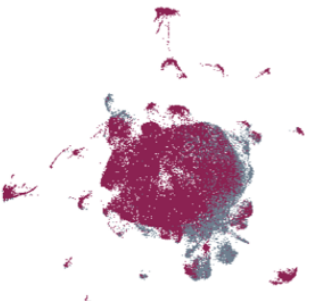

Cytokine treatment Fig. 3a cluster assignment

- Vehicle Fig. 4a Cluster 1 (IRAS1)

- Fig. 4a Cluster 2 (IRAS2)
Peripheral injection

- Saline d

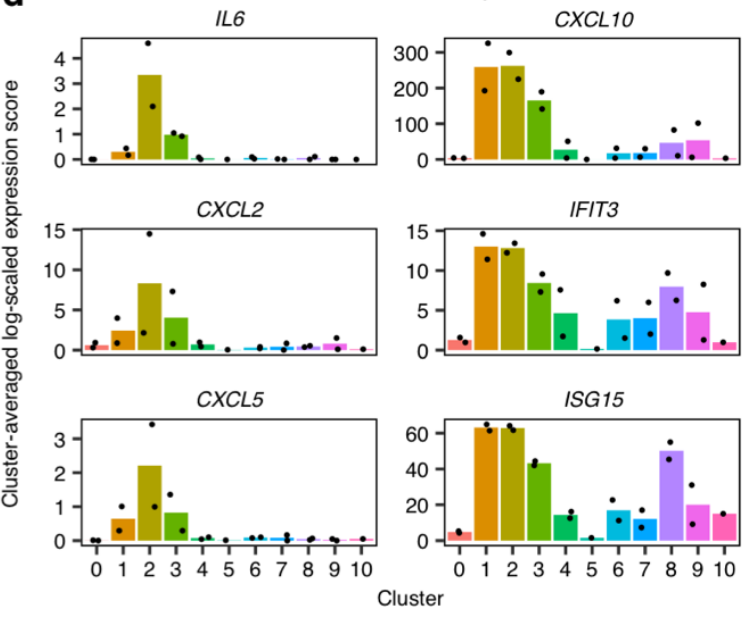

h

Wheeler et al. astrocytes
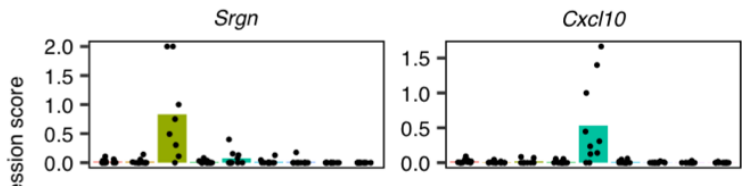

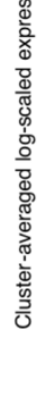
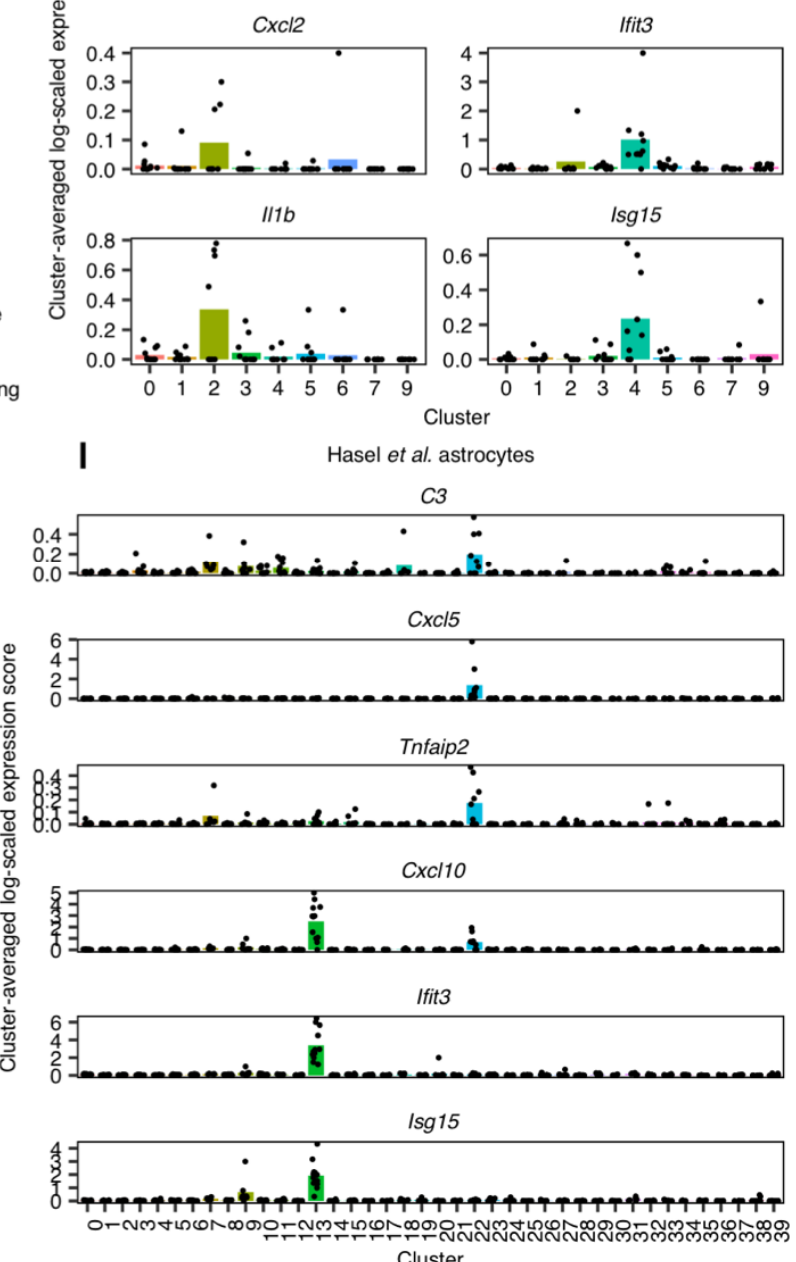

Fig. 6 (legend overleaf) 
Fig. 6 | Integration of iAstrocyte single-cell data with published single-cell datasets shows conservation of distinct inflammatory reactive states across species in diverse disease contexts. a, UMAP of integrated analysis (see Methods) of NTC sgRNA iAstrocytes with astrocytes from Barbar et al., colored by cluster assignment or astrocyte source. b-c, The same UMAP embedding as in a, showing only iAstrocytes colored by cytokine treatment or Fig. 3a cluster assignment (b) or Barbar et al. astrocytes colored by cytokine treatment (c). d, Clusteraveraged expression levels of selected cluster markers in Barbar et al. astrocytes. e, UMAP of integrated analysis of NTC sgRNA iAstrocytes with astrocytes from Wheeler et al., colored by cluster assignment or astrocyte source. f-g, The same UMAP embedding as in e, showing only iAstrocytes colored by cytokine treatment or Fig. 3a cluster assignment (f) or astrocytes from Wheeler et al. colored by EAE stage (g). h, Cluster-averaged expression levels of selected cluster markers in Wheeler et al. astrocytes. i, UMAP of integrated analysis of NTC sgRNA iAstrocytes with astrocytes from Hasel et al., colored by cluster assignment or astrocyte source. $\mathbf{j}-\mathbf{k}$, The same UMAP embedding as in $\mathbf{i}$, showing only iAstrocytes colored by cytokine treatment or Fig. 3a cluster assignment (j) or astrocytes from Hasel et al. colored by LPS treatment (k). l, Cluster-averaged expression levels of selected cluster markers in Hasel et al. astrocytes. 

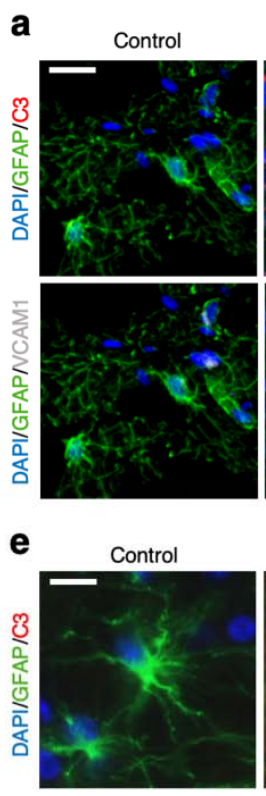

b
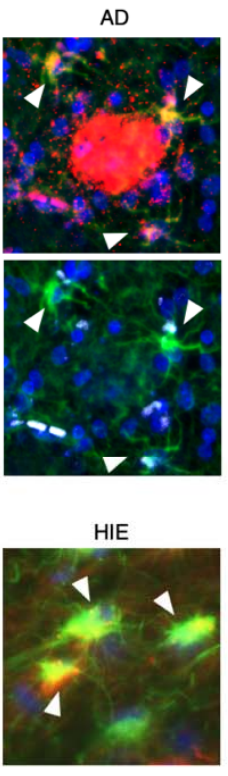
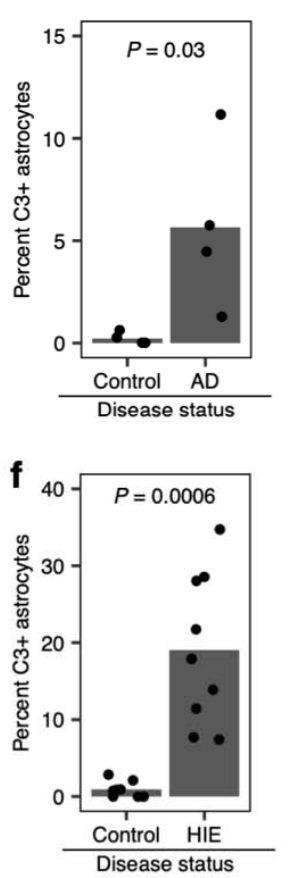

c
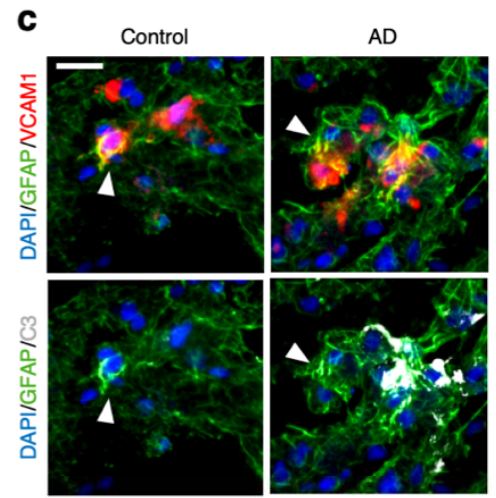

d

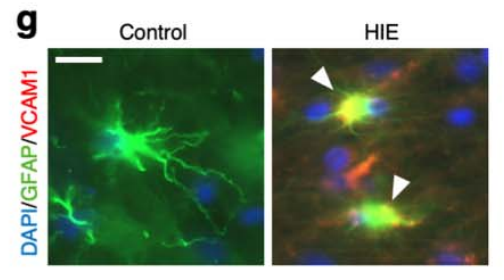

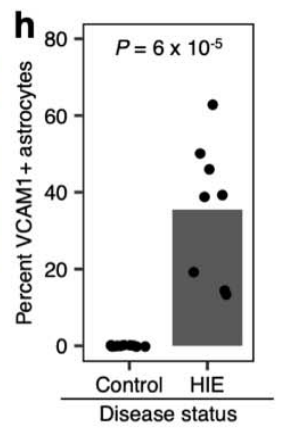

Fig. 7 | Markers of distinct inflammatory reactive states are upregulated in astrocytes in Alzheimer's disease (AD) and hypoxic-ischemic encephalopathy (HIE). a,c, Representative images of $\mathrm{C} 3+(\mathbf{a})$ or VCAM1+ (c) astrocytes (marked by arrowheads) in the AD brain compared to control; staining for VCAM1, C3, and GFAP was performed simultaneously. b,d, Quantification of the percentage of C3+ (b) or VCAM1+ (d) astrocytes in controls $(\mathrm{n}=4) \mathrm{vs}$. AD cases $(n=4)$. e,g, Representative image of C3+ $(\mathbf{e})$ or VCAM1+ $(\mathbf{g})$ astrocytes (marked by arrowheads) in HIE compared to control; staining for VCAM1 or C3 was performed separately. f,h, Quantification of the percentage of C3+ (f) or VCAM1+ (h) astrocytes in control ( $\mathrm{n}=1$ individual; data points reflect fields of view) vs. HIE $(\mathrm{n}=1$ individual; data points reflect fields of view). Scale bars in a and c correspond to $20 \mu \mathrm{m}$; scale bars in $\mathbf{e}$ and $\mathbf{g}$ correspond to $10 \mu \mathrm{m} . P$ values were calculated using the Wilcoxon ranked-sum test. 
a

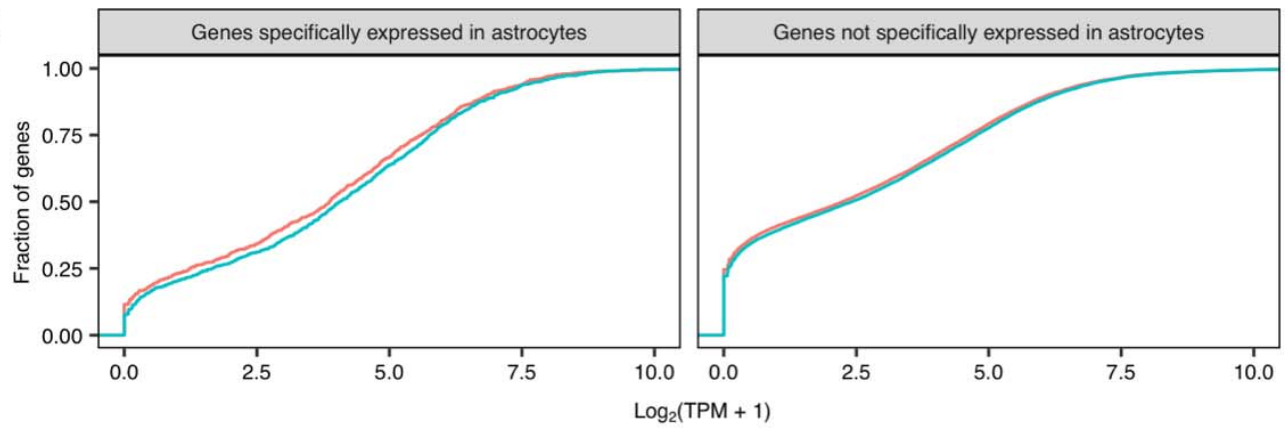

Astrocyte type

- TCW

iAstrocytes

b

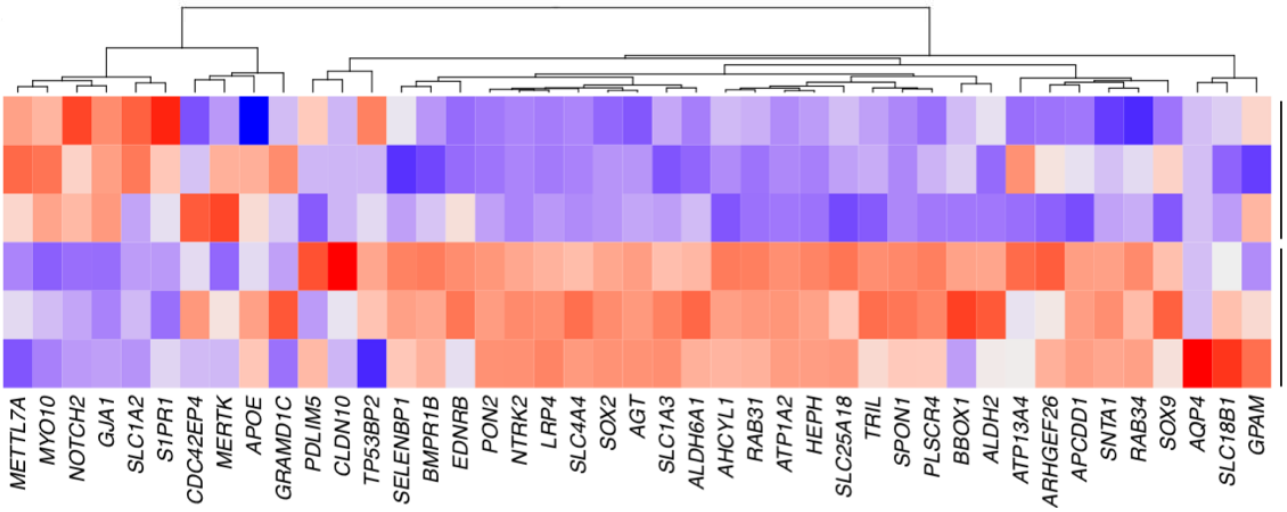

Extended Data Fig. 1 | iAstrocytes express higher levels of astrocyte-specific genes compared to iPSC-derived astrocytes generated using the protocol from TCW et al. ${ }^{18}$

a, Empirical cumulative distribution functions of the mean expression of genes (averaged across biological replicates, $n=3$ ) with astrocyte-specific expression (astrocyte fidelity $>40$ ) or without astrocyte specific expression (astrocyte fidelity $<40$ ) in iAstrocytes vs astrocytes generated using the TCW et al. protocol (TCW astrocytes). Genome-wide astrocyte fidelity scores were obtained from Kelley et al. TPM: transcripts per million. b, Relative expression (z-scored) of the top 50 genes with the highest astrocyte fidelity scores from Kelley et al. ${ }^{102}$ (organized by hierarchical clustering, see Methods) in iAstrocytes vs. TCW astrocytes ( $n=3$ biological replicates corresponding to heatmap rows). 


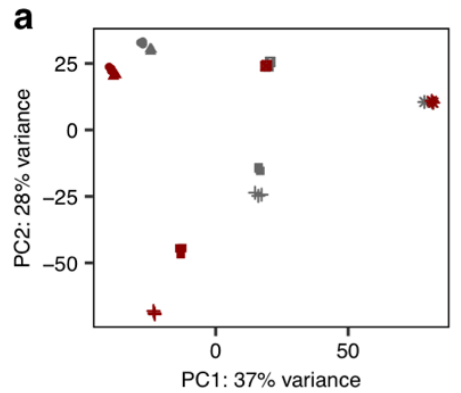

C
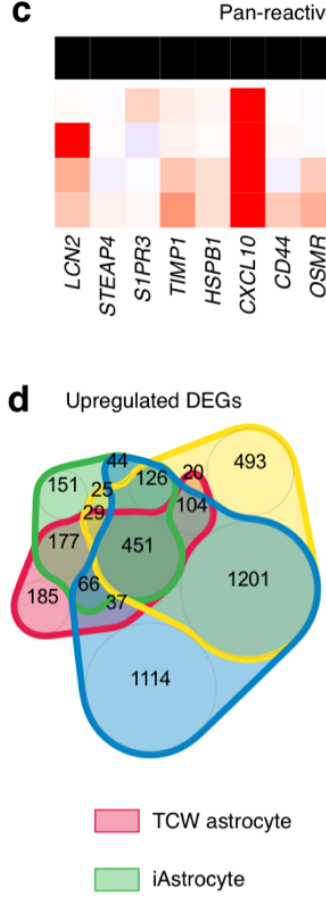

f
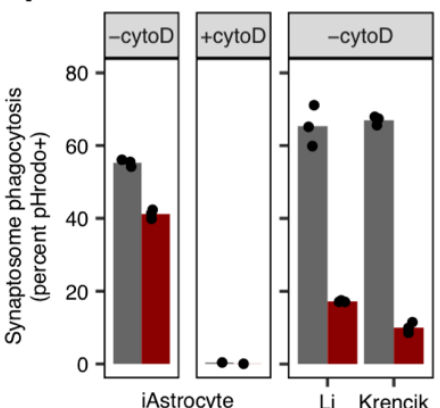

Astrocyte type

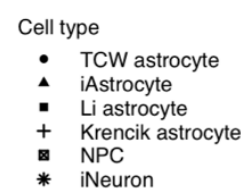

Cytokine treatment

- Vehicle

- IL-1a+TNF+C1q b

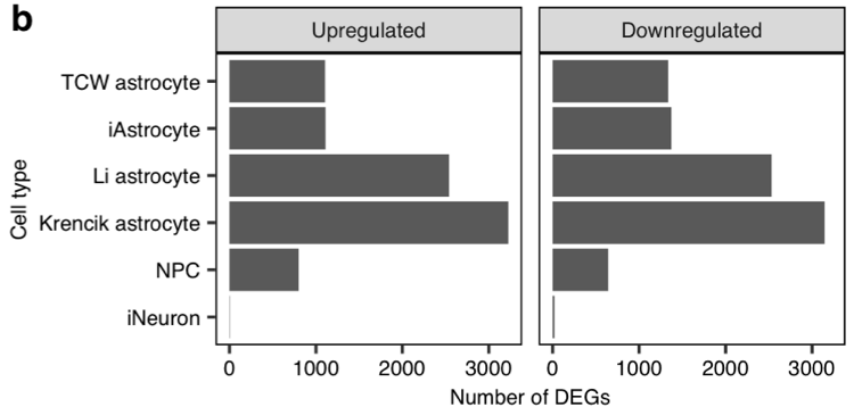

A1
A2

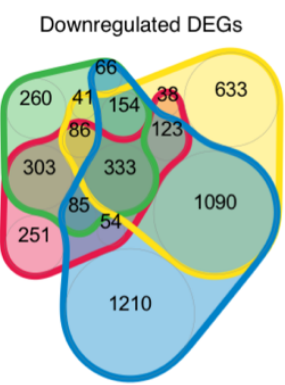

Li astrocyte

Krencik astrocyte

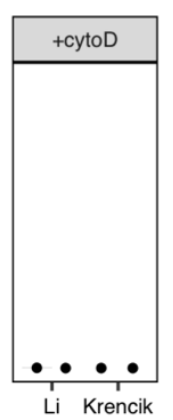

Li Krencik

g

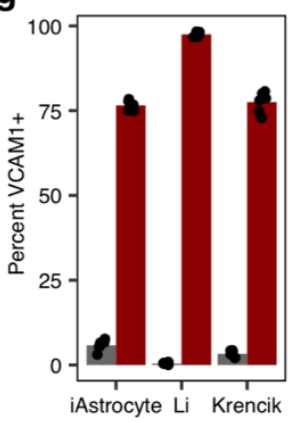

Astrocyte type

Cytokine treatment

- Vehicle

IL-1a+TNF+C1a

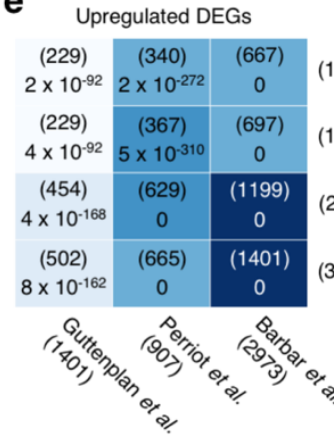

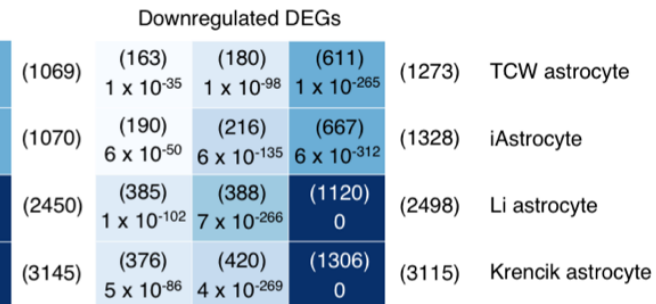

TCW astrocyte

iAstrocyte

Li astrocyte

Krencik astrocyte

$\log _{2}$ fold-change

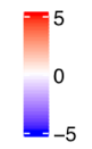

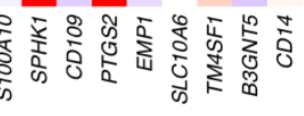

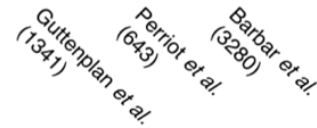

(overlap)

$P$ value

Jaccard index

$0.1 \quad 0.25$

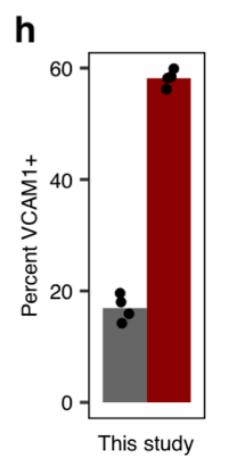

iAstrocytes

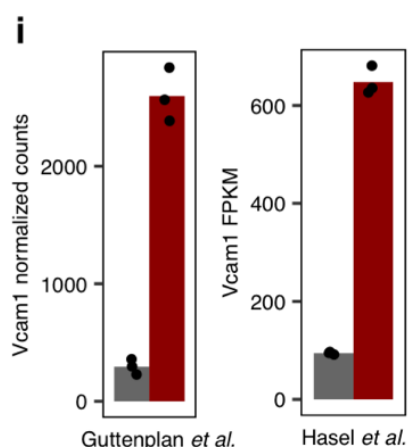

Primary mouse astrocytes

Extended Data Fig. 2 | iAstrocytes respond to IL-1 $\alpha+$ TNF+C1q in a highly similar manner as iPSC-derived astrocytes generated using different protocols and primary mouse astrocytes. a, Principal component (PC) analysis plot of the gene expression profiles (top 5000 variable genes) of iAstrocytes vs. astrocytes derived using the protocols from TCW et al. ${ }^{18}$ 
(TCW astrocytes), Li et al. ${ }^{19}$ (Li astrocytes), or Krencik et al. ${ }^{15}$ (Krencik astrocytes), as well as iPSC-derived neurons (iNeurons) and neural progenitor cells (NPCs), treated with vehicle control or IL- $1 \alpha+\mathrm{TNF}+\mathrm{C} 1 \mathrm{q}(n=3$ per condition for astrocyte samples, $n=2$ per condition for iNeuron and NPC samples). b, Number of differential expressed genes (DEGs) induced by IL$1 \alpha+\mathrm{TNF}+\mathrm{C} 1 \mathrm{q}$. c, $\log _{2}$-fold-changes of pan-reactive, $\mathrm{A} 1$ reactive, and $\mathrm{A} 2$ reactive genes defined in Liddlelow et al. ${ }^{8}$ in iPSC-derived astrocytes from this study. d, Overlap of upregulated and downregulated DEGs induced by IL- $1 \alpha+\mathrm{TNF}+\mathrm{C} 1 \mathrm{q}$ among iPSC-derived astrocytes from this study. e, Overlap of upregulated and downregulated DEGs induced by IL- $1 \alpha+\mathrm{TNF}+\mathrm{C} 1 \mathrm{q}$ from iPSC-derived astrocytes from this study compared to DEGs from inflammatory reactive astrocytes in other studies. $\mathbf{f}-\mathbf{g}$, Phagocytosis of pHrodo-labeled synaptosomes $(\mathbf{f} ; n=3$ experimental replicates per condition for-cytoD samples) or induction of cell-surface VCAM1 (g; $n=6$ experimental replicates per condition) by iAstrocytes compared to Li and Krencik astrocytes. cytoD: cytochalasin $\mathrm{D}$ ( $n=1$ per condition). $\mathbf{h}-\mathbf{i}$, Induction of VCAM1 expression by IL-1 $\alpha+\mathrm{TNF}+\mathrm{C} 1 \mathrm{q}$ in iAstrocytes measured by flow cytometry in this study (h; $n=4$ experimental replicates per condition) or primary mouse astrocytes measured by RNA-seq in Guttenplan et al. ${ }^{9}$ and Hasel et al. ${ }^{52}$ (i; $n=3$ experimental replicates per condition).
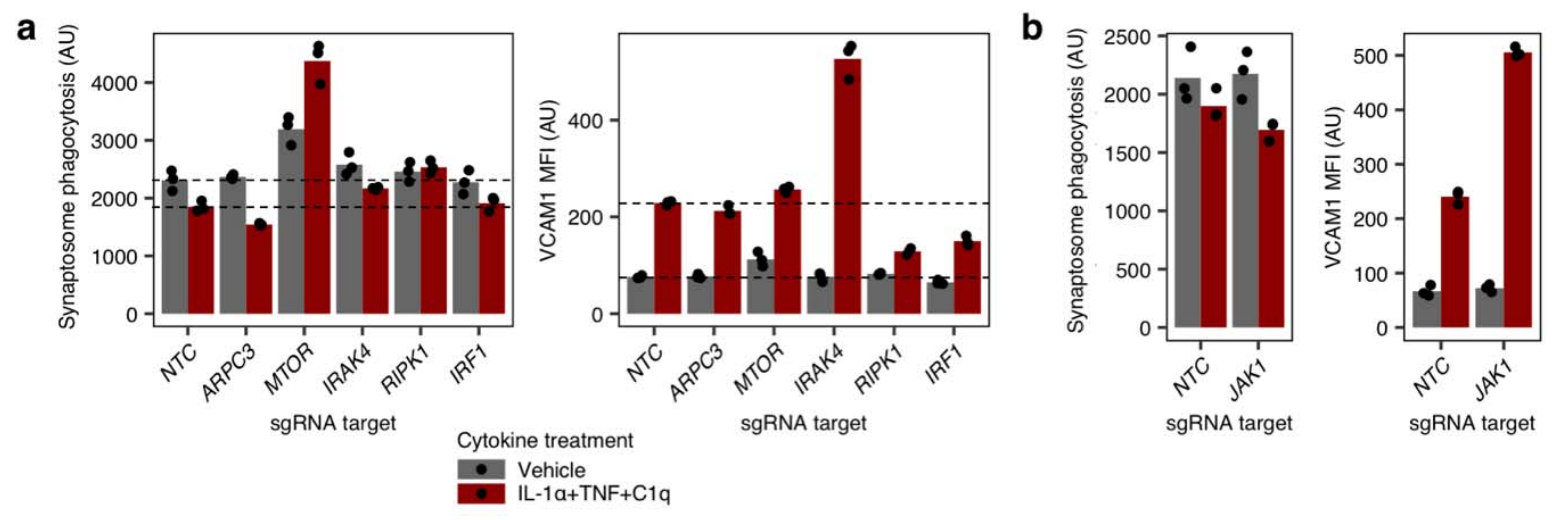

Extended Data Fig. 3 | Validation of hits from CRISPRi screens and master regulator analysis. a-b, Phagocytosis of pHrodo-labeled synaptosomes (left; median pHrodo fluorescence intensity measured by flow cytometry) or induction of cell-surface VCAM1 (right; median VCAM1 fluorescence intensity measured by flow cytometry) by iAstrocytes transduced with non-targeting sgRNA (NTC) vs. sgRNAs targeting selected top hits from the screens shown in Fig. 3, treated with vehicle control or IL-1 $\alpha+\mathrm{TNF}+\mathrm{C} 1 \mathrm{q}$. MFI: median fluorescence intensity. 
bioRxiv preprint doi: https://doi.org/10.1101/2021.08.23.457400; The copyright holder for this preprint (which was not certified by peer review)

is the author/funder, who has granted bioRxiv a license to display the preprint in perpetuity. It is made available under a CC-BY 4.0 International license .
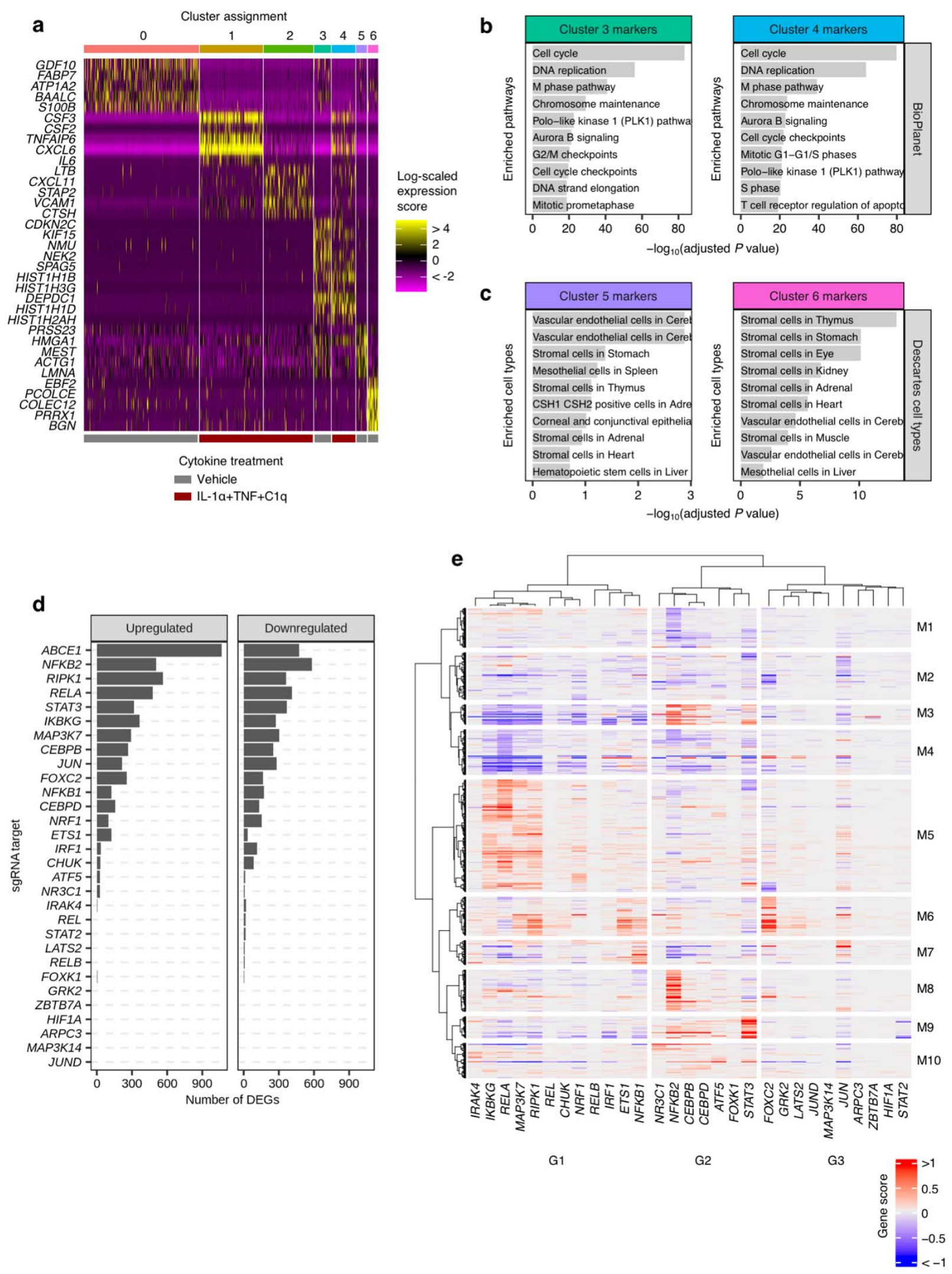

Extended Data Fig. 4 (legend overleaf) 
Extended Data Fig. 4 | Additional analyses of CROP-seq data. a, Expression levels of the top cluster markers of non-targeting control (NTC) sgRNA-transduced iAstrocytes shown in Fig. 4a. b-c, Cellular pathway (BioPlanet ${ }^{103}$ ) enrichment analysis of Cluster 3 and 4 markers (b) and cell type marker (Descartes ${ }^{104}$ ) enrichment analysis of Cluster 5 and 6 markers (c) of NTC sgRNAtransduced iAstrocytes shown in Fig. 4a. d, The number of differentially expressed genes (DEGs) whose differential expression induced $\mathrm{IL}-1 \alpha+\mathrm{TNF}+\mathrm{C} 1 \mathrm{q}$ is significantly altered by regulator knockdown. e, Hierarchical clustering of the $P$-value-weighted log-fold-changes (gene score) of the union of knockdown-associated DEGs from panel d; DEGs associated with $A B C E 1$ knockdown were excluded due to a significant number of DEGs also being caused by $A B C E 1$ knockdown in vehicle control-treated iAstrocytes. 

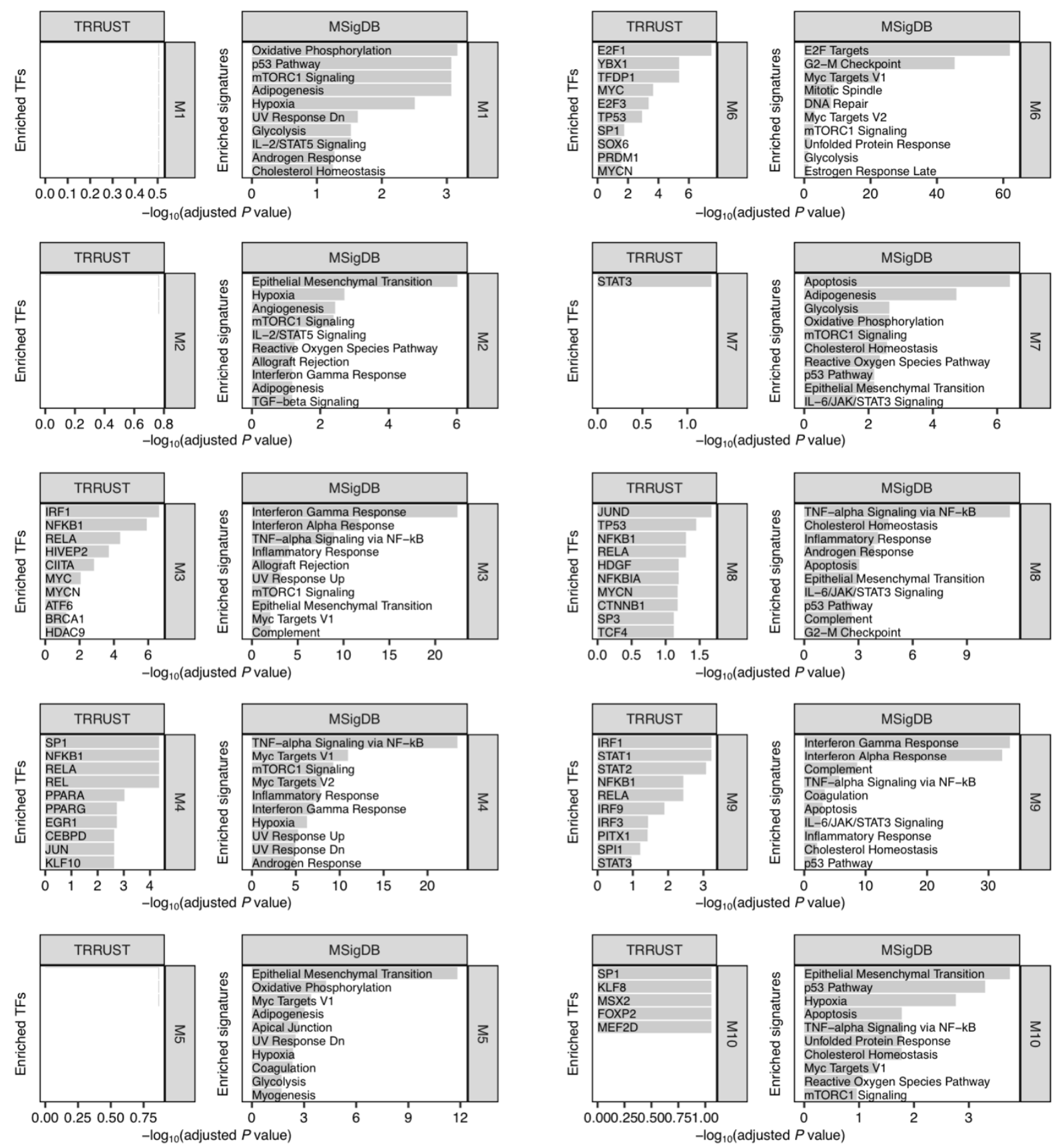

Extended Data Fig. 5 | Enrichment analysis of CROP-seq knockdown-associated gene modules. Cellular pathway $\left(\mathrm{MSigDB}^{100}\right.$ ) and upstream transcription factor (TRRUST $^{101}$ ) enrichment analysis of gene modules from Extended Data Fig. 4e; TF - transcription factor. 
a

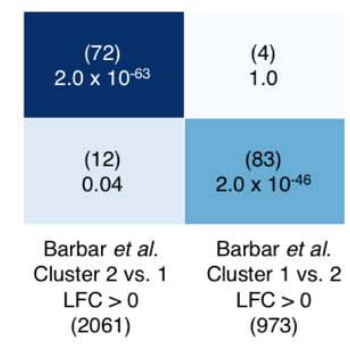

b

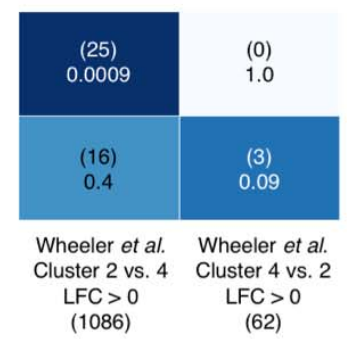

C

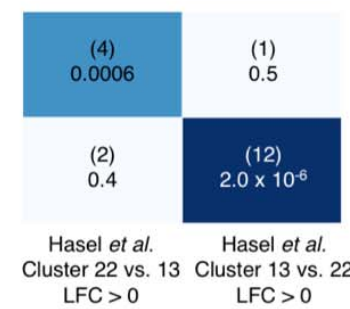

$\begin{array}{lc}\mathrm{LFC}>0 & \mathrm{LFC}>0 \\ (30) & (154)\end{array}$

d

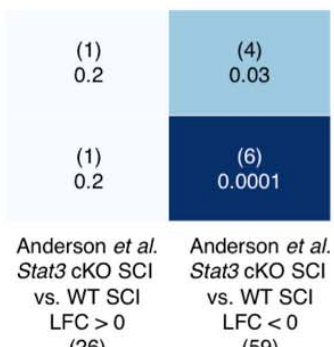

(26)

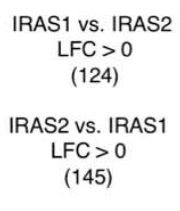
.
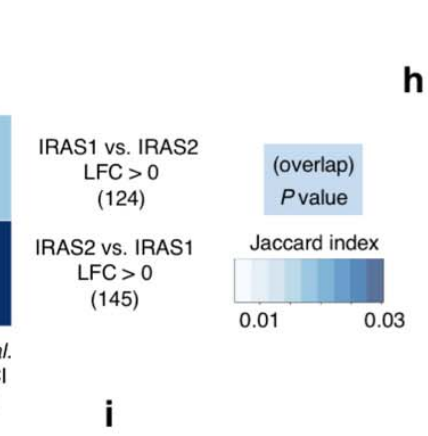

h
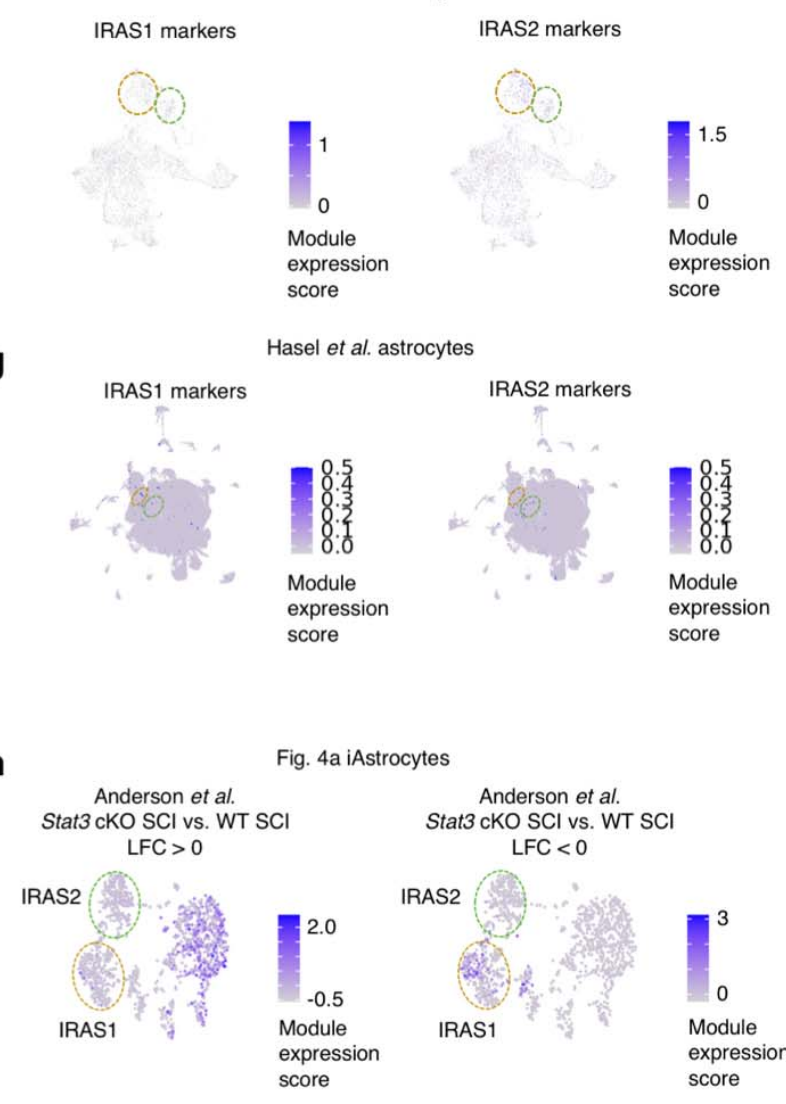

g
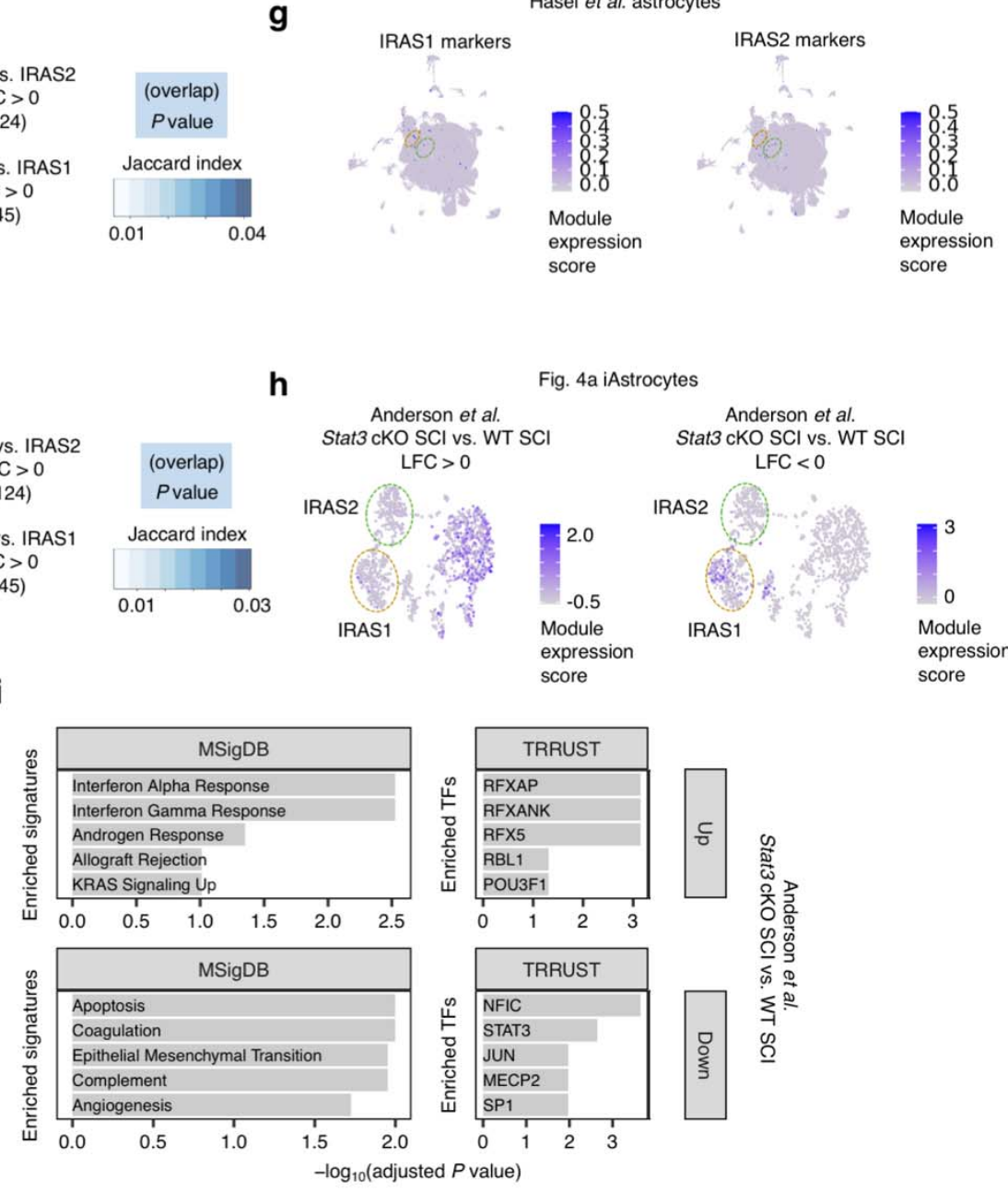

Extended Data Fig. 6 | Overlap analysis of IRAS1 and IRAS2 markers with external datasets. a-c, Overlap analysis (Fisher's exact test; see Methods) of differentially expressed genes (DEGs) between IRAS1 vs. IRAS2 with DEGs between IRAS1- and IRAS2-co-clustering 
astrocytes from Barbar et al. ${ }^{26}(\mathbf{a})$, Wheeler et $a l .{ }^{66}(\mathbf{b})$, or Hasel et al..$^{52}(\mathbf{c})$. d, Overlap analysis of DEGs between IRAS1 vs. IRAS2 with DEGs between astrocytes from Stat3 astrocyte-specific conditional knockout (cKO) mice vs. wild-type (WT) mice subject to spinal cord injury (SCI) from Anderson et $a l_{.}{ }^{45}$. e-g, Module expression score (see Methods) of IRAS1 or IRAS2 markers overlaid onto the UMAP embedding of Barbar et $a .^{26}(\mathbf{e})$, Wheeler et al. ${ }^{66}(\mathbf{f})$, or Hasel et al. ${ }^{52}$ (g) astrocytes from Fig. 7d, h, and 1, respectively. h, Module expression score of upregulated vs. downregulated DEGs between astrocytes from Stat3 cKO SCI vs. WT SCI mice from Anderson et al. $^{45}$ overlaid onto the UMAP embedding of iAstrocytes from Fig. 4a. i, Cellular pathway $\left(\mathrm{MSigDB}^{100}\right)$ and upstream transcription factor (TRRUST ${ }^{101}$ ) enrichment analysis of upregulated vs. downregulated DEGs between astrocytes from Stat3 cKO SCI vs. WT SCI mice from Anderson et al. ${ }^{45}$.

\section{SUPPLEMENTARY TABLE LEGENDS}

Supplementary Table 1| Bulk RNA-seq differentially gene expression analysis results. From this study: iAstrocytes and hiPSC-derived astrocytes generated using the protocols from TCW et al. ${ }^{18}$, Li et al. ${ }^{19}$, and Krencik et al. ${ }^{15}$ treated with vehicle vs. IL-1 $\alpha+\mathrm{TNF}+\mathrm{C} 1 \mathrm{q}$. From external datasets: immunopanned primary mouse astrocytes treated with vehicle vs. IL- $1 \alpha+\mathrm{TNF}+\mathrm{C} 1 \mathrm{q}$ from Guttenplan et al. ${ }^{9}$, hiPSC-derived astrocytes treated with vehicle vs. IL-1 $\beta$ from Perriot $e t$ $a l .{ }^{94}$, and human cerebral organoid-derived astrocytes treated with vehicle vs. IL-1 $\alpha+\mathrm{TNF}+\mathrm{C} 1 \mathrm{q}$ from Barbar $e t a l .{ }^{26}$. For data from hiPSC-derived astrocytes in this study, expression was quantified using Salmon and differentially expressed genes (DEGs) called using DESeq2. For external datasets, the DEGs from Gutteplan et al. were downloaded from the supplementary table included in GSE143598, and raw data from Perriot et al. and Barbar et al. were analyzed with BioJupies $^{93}$ to call DEGs.

\section{Supplementary Table 2 | Phenotype scores and p-values from CRISPRi screens.}

H1_phago_veh: Druggable genome library, phagocytosis screen, vehicle-treated.

H1_phago_ITC: Druggable genome library, phagocytosis screen, IL-1 $\alpha+T N F+C 1 q-$ treated. H1_VCAM1_ITC: Druggable genome library, cell-surface VCAM1 screen, IL-1 $\alpha+T N F+C 1 q-$ treated. TFs_phago_veh: Human transcription factors library, phagocytosis screen, vehicletreated. TFs_phago_ITC: Human transcription factors library, phagocytosis screen, IL$1 \alpha+\mathrm{TNF}+\mathrm{C} 1 \mathrm{q}$-treated. TFs_phago_ITC: Human transcription factors library, cell-surface VCAM1 screen, IL-1 $\alpha+$ TNF+C1q-treated. gene_TSS: gene name followed by the transcriptional start site (TSS) targeted by sgRNAs for genes with more than one TSS. geneScore: $-\log _{10}(P$ value $) *$ phenotypeScore.

Supplementary Table 3 | Hits from MRA and associated scores and statistics. See Castro et $a l{ }^{32}$ for interpretation of the activity score. The mean LFC score of a regulon is calculated by averaging the $\log _{2}$-fold-change of all differentially expressed genes induced by IL- $1 \alpha+\mathrm{TNF}+\mathrm{C} 1 \mathrm{q}$ in that regulon.

\section{Supplementary Table 4 | Cluster markers of iAstrocytes in Fig. 4a and differential expression analysis between IRAS1 and IRAS2 iAstrocytes. The column "avg_diff" contains}


the magnitude of the difference in the mean Pearson residual values of a given gene between cells in the cluster of interest vs. all other cells, or between IRAS2 (cluster 2) vs. IRAS1 (cluster 1) iAstrocytes. The columns "pct.1" and "pct.2" contain the percent of cells expressing the gene of interest in the cluster of interest ("pct.1") or all other cells ("pct.2"), or in IRAS2 ("pct.1") iAstrocytes or IRAS1 (“pct.2”) iAstrocytes.

Supplementary Table 5 | Enrichment analysis of IRAS1 and IRAS2 markers. Enrichment results for the MSigDB ${ }^{100}$ and TRRUST ${ }^{101}$ libraries were downloaded from Enrichr ${ }^{95}$. See Chen et al. ${ }^{95}$ for how the "combined score" is calculated.

Supplementary Table 6 | Differential gene expression analysis results from knockdown of regulators in CROP-seq experiment. The results shown correspond to the interaction term between cytokine treatment and regulator knockdown, i.e. they reflect how regulator knockdown affects the change in gene expression induced by $\mathrm{IL}-1 \alpha+\mathrm{TNF}+\mathrm{C} 1 \mathrm{q}$.

Supplementary Table 7 | Gene modules and enrichment analysis of gene modules from Extended Data Fig. 4d. Enrichment results for the MSigDB ${ }^{100}$ and TRRUST ${ }^{101}$ libraries were downloaded from Enrichr ${ }^{95}$. See Chen et al. ${ }^{95}$ for how the "combined score" is calculated.

\section{Supplementary Table 8 | Cluster markers and DEGs from integrated analysis of} iAstrocytes with Barbar et al., Wheeler et al., or Hasel et al. astrocytes. Cluster markers and DEGs are derived using only astrocytes from Barbar et al. ${ }^{26}$, Wheeler et al. ${ }^{66}$, or Hasel et al. ${ }^{52}$. The column "avg_diff" contains the magnitude of the difference in the mean Pearson residual values of a given gene between cells in the cluster of interest vs. all other cells, or between two clusters of interest (the first cluster minus the second cluster). The columns "pct.1" and "pct.2" contain the percent of cells expressing the gene of interest in the cluster of interest ("pct.1") or all other cells ("pct.2"), or the two clusters of interest.

Supplementary Table 9 | Differential gene expression analysis between Stat3 cKO SCI vS. WT SCI astrocyte transcriptomes from Anderson $e t$ al. and enrichment analysis of DEGs. The raw data from Anderson et $a .^{45}{ }^{\text {was analyzed with BioJupies }}{ }^{93}$ to call DEGs between Stat 3 cKO SCI vs. WT SCI astrocyte transcriptomes. Enrichment results for the MSigDB ${ }^{100}$ and TRRUST ${ }^{101}$ libraries were downloaded from Enrichr ${ }^{95}$. See Chen et al. ${ }^{95}$ for how the "combined score" is calculated.

\section{Supplementary Table 10 | Clinical metadata of human neuropathology samples.}

Supplementary Table 11 | Metadata of astrocyte bulk RNA-seq samples used for coexpression network reconstruction in master regulator analysis. Samples from publicly available datasets can be cross-referenced with GEO using their GSM accession numbers. 